\title{
Nonlinear transient and steady state analysis for self-excited single-phase synchronous reluctance generator
}

Jingdong Chen

West Virginia University

Follow this and additional works at: https://researchrepository.wvu.edu/etd

\section{Recommended Citation}

Chen, Jingdong, "Nonlinear transient and steady state analysis for self-excited single-phase synchronous reluctance generator" (2001). Graduate Theses, Dissertations, and Problem Reports. 1383.

https://researchrepository.wvu.edu/etd/1383

This Dissertation is protected by copyright and/or related rights. It has been brought to you by the The Research Repository @ WVU with permission from the rights-holder(s). You are free to use this Dissertation in any way that is permitted by the copyright and related rights legislation that applies to your use. For other uses you must obtain permission from the rights-holder(s) directly, unless additional rights are indicated by a Creative Commons license in the record and/ or on the work itself. This Dissertation has been accepted for inclusion in WVU Graduate Theses, Dissertations, and Problem Reports collection by an authorized administrator of The Research Repository @ WVU.

For more information, please contact researchrepository@mail.wvu.edu. 


\title{
Nonlinear Transient and Steady State Analysis for Self-excited Single-phase Synchronous Reluctance Generator
}

\author{
By Jingdong chen \\ Dissertation submitted to the \\ College of Engineering and Mineral Resources \\ at West Virginia University \\ in partial fulfillment of the degree of \\ Doctor of Philosophy \\ in Electrical Engineering
}

Academic and Examining Committee Members:

Parviz Famouri, Ph.D., Chair

Muhammad Choudhry, Ph.D.

Ronald Klein, Ph.D.

Larry A. Hornak, Ph.D.

Ian Christie, Ph.D.

Department of Computer Science and Electrical Engineering

Morgantown, West Virginia

2001

Keywords: Single-phase, Self-excited, reluctance generator, Nonlinear analysis

Copyright 2001 Jingdong Chen 


\title{
ABSTRACT
}

\section{Nonlinear Transient and Steady State Analysis for Self-excited Single-phase Synchronous Reluctance Generator}

\author{
By Jingdong chen
}

With today's trend for distributed generation and the need for alternative and renewable energy sources, self-excited induction and synchronous reluctance generators have attracted more attention for wind, tidal and hydro power generation applications. Compared to synchronous and DC generators, they have the advantages: they are brushless, they are robust, they do not need DC excitation and they are relatively low cost.

Compared with SEIG, the self-excited reluctance generator (SERG) not only has the advantages of simplicity and ruggedness, but can also have enhanced steady-state characteristics and high efficiency over a wide range of operation. Moreover, its output frequency is determined only by the prime mover speed, rather than by both the load and the prime mover speed as in an induction generator, so SERG can be easily integrated with power electronic devices to implement a control scheme.

Most of the current analyses deal with three-phase reluctance generators, but insufficient attention has been paid to single-phase self-excited reluctance generators (SPSERG). Their unbalanced loads make their analysis more difficult. This research is motivated by the fact that SPSERG provides a good alternative to single-phase induction generators used in stand-alone generation applications. A general methodology is suggested for transient response prediction and steady state performance analysis for the SPSERG type of electric machine.

To establish a design environment, finite element method is an effective tool, which can be integrated in machine modeling to obtain good performance prediction. In this work, an off-line FEM approach is proposed to obtain the saturation characteristics for state space simulation. During the process, transformation between instantaneous inductance and average inductance is investigated. Off-line FEM+SS approach is proved to be a simple and economic method and can fit the experimental results in good accuracy.

Moreover, a steady state model has to be built to reveal the parametric dependence and provide good design guidance. However, because of the unbalanced load and nonlinear feature of the machine, existing models are not suitable for analysis. In this dissertation, a novel inductance-oriented steady state model based on the harmonic balance technique is introduced. The idea is that starting from the inductance determination under certain load, the fluxes can be attained by a nonlinear relationship, after that, the machine variables can be solved according to the fluxes. Comparison between simulation and experiment validates this approach. 


\section{Acknowledgement}

I would first like to thank my advisor and academic committee chairman, Dr.

Parivz Famouri, for his support, guidance and encouragement throughout this research. I would also like to express my appreciation to Dr. Muhammad Choudhry, Dr. Ronald

Klein, Dr. Larry A. Hornak and Dr. Ian Christie for serving on my examining committee and for their constructive advice.

I would especially like to thank my wife and daughter for their love and support. Without them by my side, I cannot have gone through so many hard times during my Ph.D. program.

I would also like to thank my parents-in-law. Their sacrifice and support have made it possible for me to concentrate on my work and to finally complete this dissertation.

No words can express my thanks to my parents. They taught me how important education is. They also taught me to be a man who never gives up. Their spiritual support and expectation are always my great propulsion to success. 


\section{Table of Contents}

Title Page

Abstract

Acknowledgements

Table of Contents

Chapter 1 Introduction 1

$1.1 \quad$ Background 1

1.2 Problem Identification 3

$\begin{array}{lll}1.3 & \text { Literature Review } & 9\end{array}$

1.3.1 Equivalent Circuit Modeling 9

1.3.2 Finite Element Plus Equivalent Circuit Modeling $\quad 10$

$\begin{array}{lll}1.4 & \text { Research Objectives } & 12\end{array}$

$\begin{array}{lll}1.5 & \text { Contributions } & 14\end{array}$

Chapter 2 System Modeling 15

2.1 System Model under ABC Natural Frame for Resistive Load 15

2.2 System Model under QD Reference Frame for Resistive Load 17

2.3 System Model under ABC Natural Frame for RL Load 21

2.4 System Model under QD Reference Frame for RL Load 23

$\begin{array}{lll}\text { Chapter } 3 \quad \text { Magnetic Model } & 27\end{array}$

$\begin{array}{lll}3.1 & \text { Introduction } & 27\end{array}$

3.2 FE Modeling for SPSERG 30

3.3 Obtaining the Inductance 35

Chapter 4 Transient Analysis Using Off-line FE+SS Method 37 
4.1 Measuring the Inductance 38

4.2 Obtaining Inductance by FEM 39

4.3 Transformation 42

$\begin{array}{lll}\text { Chapter } 5 & \text { System Steady State Analysis } & 47\end{array}$

5.1 Harmonic Balance Technique 47

5.2 New Steady State Model Using Harmonic Balance Technique 49

5.2.1 Stator Model 51

5.2.2 Terminal Model with Resistive Load 52

5.2.3 Terminal Model with RL Load 53

5.3 Proposed Algorithm 54

5.4 Reconstruction of the State Variable's Waveform 56

Chapter $6 \quad$ Simulation and Experiment Results $\quad 59$

6.1 Simulation and Experiment for Transient Analysis 60

6.1.1 Simulation Using the Measured Saturation Curves 60

6.1.2 Inductance Profile Analysis 64

6.1.3 Transient Simulation Using Computed Saturation Curves 67

6.2 Simulation and Experiment for Steady State Analysis 73

6.2.1 Waveform Analysis 73

6.2.2 Performance Analysis 78

6.2.2.1 Resistive Load 79

6.2.2.2 RL Load 82

6.2.3 Error Sources for Steady State Analysis 85

$\begin{array}{lll}\text { Chapter } 7 & \text { Conclusion } & 90\end{array}$

7.1 System Modeling and Simulation for Transient Analysis 90

7.2 Steady State Model Development and Simulation 92

$\begin{array}{lll}7.3 & \text { Future Work } & 93\end{array}$ 
Bibliography $\quad 95$

$\begin{array}{lll}\text { Appendix I Program Code } & 98\end{array}$

$\begin{array}{ll}\text { Vita } & 128\end{array}$

Approval of Examining Committee 132 


\section{CHAPTER}

\section{ONE}

\section{INTRODUCTION}

\subsection{Background}

With today's trend for distributed generation and the need for alternative and renewable energy sources, self-excited induction and synchronous reluctance generators have attracted more attention for wind, tidal and hydro power generation applications. Compared to synchronous and DC generators, they have the advantages of brushless, robustness, low cost with no need for a DC excitation.

There has been substantial research conducted in system modeling and control schemes of these generators, especially for induction types. Self-excited induction generator (SEIG) was one of the earliest types of self-excited AC generator and has its inherent advantage of low unit price, robust, brushless structure with squirrel-cage rotor, reduced size, no DC excitation and better transient performance, etc. However, both magnitude and frequency of the output voltage and current are load dependent, which makes performance prediction difficult and complicates the control strategy.

Compared with SEIG, self-excited reluctance generator (SERG) not only has the advantages of simplicity and ruggedness, but can also give high efficiency over a wide range of operation [1][2]. Moreover, its output frequency is determined only by the prime 
mover speed, rather than by both load and the prime mover speed as in an induction generator, so it can be easily integrated with a power electronic converter to control the output [3].

Furthermore, at the same power output level, for permanent magnet synchronous generator, aging of the magnets at elevated temperature may totally demagnetize the magnets. Such problem can also happen under high armature reaction on load, or shortcircuit fault. For the reluctance generator, the operating temperature is limited only by insulation. So with appropriate design consideration, the power/weight ratio can be improved and comparable to permanent synchronous generator. Moreover, with appropriate compensation techniques using inverter-battery supply connected with the load, performance can be greatly improved [4]. These merits outweigh its disadvantage of low iron utilization.

The operation of SERG depends on flux saturation. So the usual analysis and design methods, which are based on the assumption of infinite permeability of the iron, can only predict the characteristic for its unsaturated operation. It is the purpose of this study to provide a method for the analysis of the SERG taking saturation of the iron into account.

Most of the current analyses deal with three-phase reluctance generators, but sufficient attention has not been paid to single-phase, self-excited, reluctance generators (SPSERG). Their unbalance loads make the analysis cumbersome. Since they are good alternatives to single-phase induction generators used in stand-alone generation applications, SPSERG is investigated in this research and a general methodology is 
suggested for transient response prediction and steady state performance analysis for this type of electric machine.

\subsection{Problem Identification}

As shown in Figure 1-1, SPSERG in this study is consisted of a salient rotor with two poles, stator with 18 slots, and concentric stator main and auxiliary windings with sinusoidal distribution. The winding layout is in Table I.

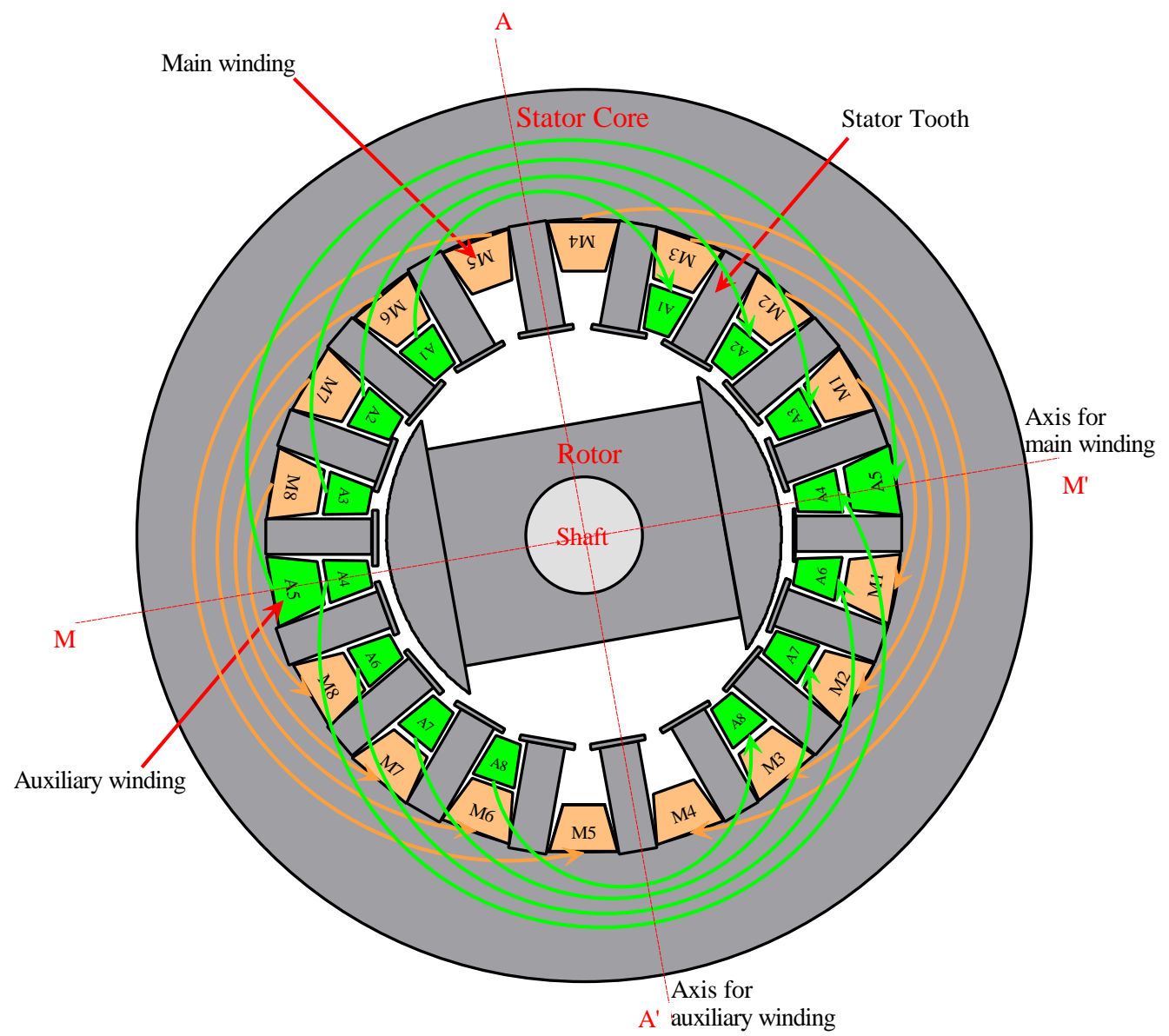

Figure 1-1: Cross section view of single-phase reluctance generator. 
Table I: Winding layout

\begin{tabular}{|c|c|c|c|}
\hline Main Winding & Turns & Auxiliary Winding & Turns \\
\hline M1, M8 & 9 & A1, A8 & 13 \\
\hline M2, M7 & 17 & A2, A7 & 21 \\
\hline M3, M6 & 23 & A3, A6 & 25 \\
\hline M4, M5 & 26 & A4, A5 & 14 \\
\hline Total & 150 & Total & 146 \\
\hline
\end{tabular}

The terminal connections for the SPSERG are shown in Figure 1-2. One capacitor $C_{a}$ is connected with the auxiliary winding, while another capacitor $C_{m}$ is parallel connected with the load to the main winding.

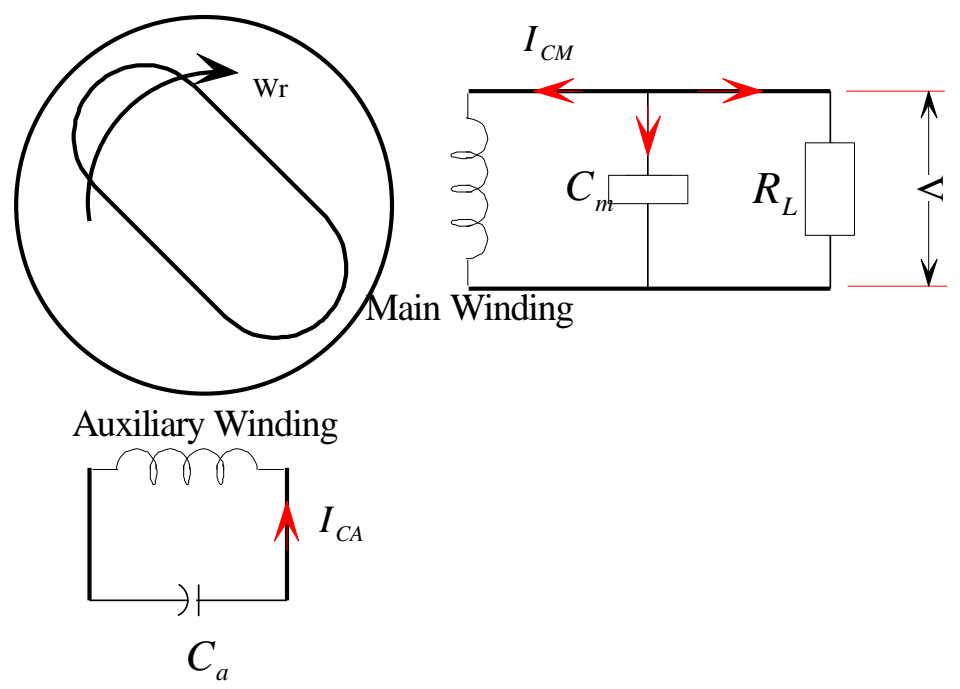

Figure 1-2: Schematic of the generator

Stable operation of the SPSERG depends on a self-excitation process, as shown in Figure 1-3. Self-excitation of a generator begins by the action of either a residual air gap flux linkage $\Phi_{r}$ or charge on the excitation capacitors. This residual flux linkage $\Phi_{r}$ 
with the move of the rotor develops an induced voltage $V_{r}$ in the stator winding. Because of the capacitance, two leading current $I_{C M}$ and $I_{C A}$ in the main and auxiliary windings are generated, which in turn strengthen the field represented as $\Phi_{C}$. This flux and the rotor movement then produce a higher induced voltage $V$.

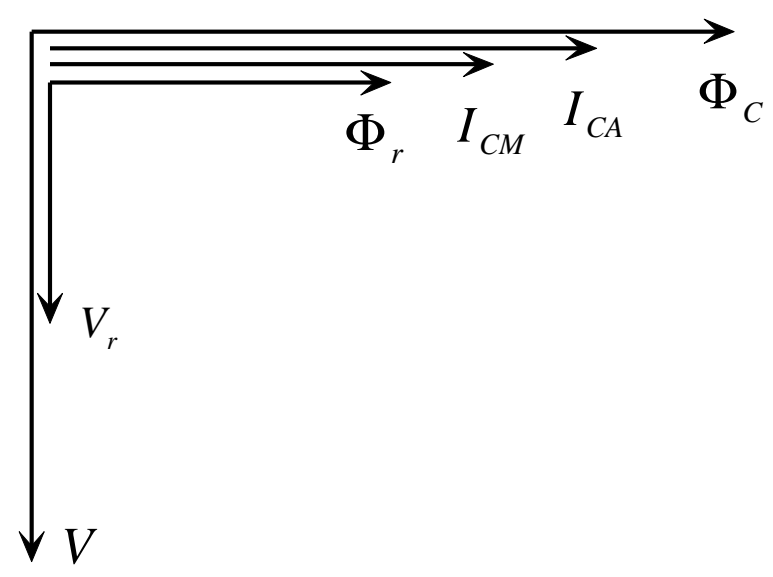

Figure 1-3: Phasor diagram

With sufficient capacitance, the process continues leading an increase in induced stator voltage until it settles to a steady state operating condition determined by the air gap flux linkage saturation as illustrated in Figure 1-4.

Due to its nonlinear nature, the transient and steady state behavior of the SPSERG largely depends on the magnetic saturation. In order to predict the transient selfexcitation process and steady state performance accurately, a nonlinear model, which can deal with saturation, is worth investigating. 


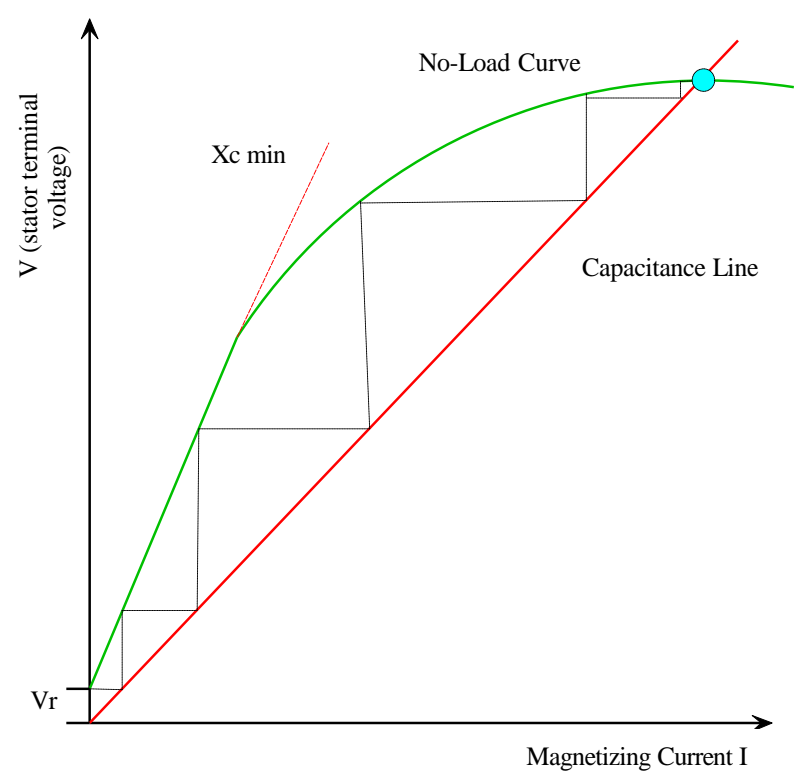

Figure 1-4: Self-excitation process

In most of the prior work on the induction and synchronous reluctance machine modeling, the parameters which mainly are the inductances are measured from the steady state test. And the saturation is considered by polynomial data fitting function. However, from design point of view, it will be more desirable if the machine performance can be predicted before the prototype is manufactured.

In conventional analysis of the reluctance machine, assumptions include even distribution of the flux density and balanced load. For single-phase reluctance machine, however, the load is only connected with main winding, which makes the main and auxiliary winding's terminal connections unbalanced. So if unbalance condition is considered, both transient and steady state analyses will be complicated.

This dissertation will show that it is more suitable to use $A B C$ reference frame rather than QD reference frame to solve the transient process. ABC reference frame can include various harmonics which results in improving the solution accuracy. In steady 
state analysis, on the other hand, QD reference frame is more suitable because of its time invariant inductances.

Since no external electrical sources are applied, no information about the air gap field can be attained. As a result, conventional steady state analysis cannot be directly used in this case. On the contrary, a backward analysis is more appropriate, which means given the prime mover speed, load resistance and terminal capacitance, the steady state air gap flux has to be solved first, then relevant output variables can be attained.

Another problem in SPSERG is the apparent torque ripple, which is generated by the unbalance load and rotor saliency. In steady state analysis of SERG, most authors just set the time-derivative terms in the QD model to zero, then use the algebraic equations to determine the performance. Assumption in the approach is that the state variables in QD model are constant, which is not true in SPSERG under unbalance load. Techniques such as the harmonic balance technique has to be applied to overcome this problem.

Actually, there are unbalance analyses for synchronous generators set forth by Krause [5] and R.J.Kerman [6], but they cannot be directly used in this case. First, the methods can only deal with the linear model and saturation is not considered, which is an adequate assumption for three-phase synchronous generators. Second, the main concerns are unbalance power supply, such as line to line or line to neutral faults, which are unbalance operation, not unbalance load.

To accurately predict SPSERG performance, finite element method should be used to solve the field. The application of the finite element method for the analysis of electrical machines has long been recognized, and a vast literature has been published dealing with most of the existing machines. The advantages of this approach are their 
ability to model the whole structure within the machine and the ability to model magnetic saturation to a high accuracy. Analyses with finite element method have been carried out not only to observe the flux distribution, but also to use the results more effectively to achieve high design accuracy and dynamic modeling capabilities.

In this study, machine magnetic saturation determines the transient response and the steady state operation. Therefore, appropriate consideration of the nonlinear characteristics is essential and high accuracy of the magnetic saturation description is important for performance analysis. To get a more accurate saturation profile of the inductances and to better predict the machine performance, the finite element method is used. Moreover, in conjunction with state space equations under the $\mathrm{ABC}$ reference frame, machine performance is predicted in the transient process with a good accuracy.

This dissertation is organized as follows. Chapter 2 sets forth mathematical models in $\mathrm{ABC}$ reference frame and QD reference frame considering different type of loads. Finite element method (FEM) is introduced and magnetic model of the SPSERG is provided in Chapter 3. Then an off-line FEM+SS method for transient analysis is given and discussed in Chapter 4. Chapter 5 proposes a novel steady state method for unbalance load analysis basing on harmonic balance. Simulation and experimental results are shown in Chapter 6 to illustrate the effectiveness of the proposed methods for both transient and steady state analyses. Conclusions are given in Chapter 7.

\subsection{Literature Review}

The reluctance machines are strong competitors to induction machines in many applications such as high performance drives and auxiliary or isolated power generating 
systems. Compared with induction generator, desirable characteristics of the reluctance generators include their simple construction, low cost, small rotor lose, lightweight, low inertia [1-3].

\subsubsection{Equivalent Circuit Modeling}

Most of the papers in this area concern with the steady state performance, its load and excitation capacitance effect on terminal voltage, power factor, and stability [7][811]. Others focus on its self-excitation and de-excitation process to predict the transient. However, both the transient and steady state analyses are based on QD reference frame and a measured saturation curve.

Olorunfemi Ojo [8] uses the QD model to analyze the transient and steady state performances of reluctance generator. Small signal and bifurcation analyses are utilized to explain the de-excitation phenomenon. Saturation of the inductances is lumped to one inductance and represented by a polynomial. In another paper [9] of this author, unbalance winding distribution and unequal load condition are considered for a motor operation. Saturation is involved by measured nonlinear inductance curves. The dependence of machine parameters and capacitor value on the transient torque and current is investigated by an asynchronous model.

T.F. CHAN [10] presents a general method for analyzing the steady state performance of a three-phase, self-excited, reluctance generator with RL load. Magnetic saturation is assumed to be confined to the direct-axis and is accounted for by a variable direct-axis magnetizing reactance that satisfies the circuit conditions. Measured saturation curve is also used. 
L. Wang and Y. Wang [11] introduce an Eigenvalue and Eigenvalue sensitivity method to obtain the minimum loading resistance. In their paper, three-phase SERG is represented by a QD model the same way as is in an induction machine, from which some doubts could arise. Since it infers that the reluctance machine works asynchronously, and can only be valid if the rotor has enough poles.

For unbalance condition, only A.H. AlBahrani, N.H. Malik [12] investigate the steady state performance for SEIG with a single capacitor and single phase load. And L. Wang, R. Deng [13] examine the transient response for the same problem. ABC reference frame is applied in both papers. Because of the time invariant inductance for induction generators, equivalent circuits based on double-revolving field theory are used to solve the steady state characteristics. However, it is not easy to extend this work to SERG because of the time variant inductance, so new methods have to be developed.

\subsubsection{Finite Element plus Equivalent Circuit Modeling}

All the methods used in the above papers use equivalent circuit model only, and the saturation effect is considered by an analytical expression.

If more accurate estimation is required, comprehensive measurements or finite element method will be needed. However, from design point of view, it is more appropriate to predict the saturation before any prototype is built, so a field analysis method such as finite element method should be the choice. To integrate finite element method into the machine analysis, immense endeavor has been made for synchronous and induction machines. Among all the existing methods, the time stepping technique is widely used to solve dynamic problems where the solution depends on both the variation 
of the magnetic field in time and on saturation. The method offers the possibility to update circuit parameters and therefore gives accurate analysis of machine performance. Drawback of this approach is that it is very time consuming and is normally implemented on main frame computer.

For transient analysis, A.C.Smith and et al [14] introduce a time-stepping method to predict currents and torques in wounded-rotor induction motor using FEM. The circuit and field equations are directly coupled and winding inductances are updated to account for slotting and saturation effects. And the mechanical dynamic equation is also embedded in the analysis. This approach is also applied in $[15,16,17]$. Even though it is really attractive for its good accuracy, the apparent disadvantage is that it requires great computing time and resources.

Deng F. and Demerdash, N.A. $[18,19,20,21]$ and P. Baldassari [22] propose a coupled finite-element state space approach $(\mathrm{CFE}+\mathrm{SS})$ to construct the state variable's waveforms under steady state, based on the natural $\mathrm{ABC}$ flux-linkage frame of reference, to predict a salient-pole synchronous generator's performance, including damper bar currents and iron core losses. The effects of space harmonics and time harmonics are included in the simulation. However, this method can only deal with steady state performance and the computation is still very intensive. In addition, it can only work on system with forcing functions, such as $\dot{X}=A X+B U$. But for self-excited system like SERG with no forcing function, system equation is $\dot{X}=A X$. The operation point heavily depends on the saturation, and the inductance must be evaluated at every time step, otherwise, no steady state can be reached. 
B. Sarlioglu and T.A. Lipo [23] suggest a FEM method for modeling a salient nonlinear machine. They consider the flux as a function of both position and stator current, and find their relationship by applying FEM at different rotor position and different stator current. Next, cubic spline interpolation technique is used to get an analytic expression. Then, expressions for the partial derivatives of the flux with respect to position and current are prepared for circuit simulation. This approach is appealing for its simplicity. But in their case, only one winding is on the stator side. If there are two windings, flux function is a three variable function, which will increase the computation effort exponentially.

\subsection{Research Objectives}

The limitation of existing equivalent circuit methods is that they base on measured saturation curve and loads are balanced three-phase loads. Therefore, when unbalance loads are introduced, the existing methods cannot be applied directly. A new method has to be proposed. Moreover, a prototype has to be built to measure the saturation curve, which is costly and not desirable for a design environment.

On the other hand, even though FEM plus equivalent circuit model can provide accurate transient and steady state analysis, the computation effort is too high to be used for a simple generator design.

To solve a more general nonlinear problem with unbalance load, new approaches are proposed in this dissertation for transient and steady state analyses.

The research objectives of this work can be summarized as following:

(1): Off-line FE+SS approach is implemented for the transient simulation 
considering the nonlinear characteristics of materials.

(2): A steady state method using harmonic balance technique [25] is introduced to predict the machine performance. Simulation results will be compared with the experiment results.

(3): Match the experimental steady state output voltage waveform with the simulation result. Also match the self-excitation process from the simulation with the measured response.

\section{Assumptions:}

The following assumptions are made for this study and maybe included as a part of future research.

(1): Comprehensive investigation of minimum capacitance requirement and optimal capacitance arrangement for $C_{m}$ and $C_{a}$ for the self-excitation is not examined, because the purpose of this research is concerned with the validation of novel stead state analysis for unbalance load.

(2): Space harmonics of the stator inductances are neglected for simplicity.

\section{$\underline{1.5 \text { Contributions }}$}

The contributions of this dissertation are:

(1): It is the first time with the author's knowledge, that off-line FEM+SS coupled approach is used for self-excited type AC generators, which provides a simple technique for machine design and analysis for transient and steady state performances. Compared with existing methods that are only able to be solved by a main-frame computer, this 
combined method can be easily implemented on a PC workstation, which eases the high intense computation effort.

(2): Moreover, based on harmonic balanced technique, a steady state analysis method for electric generators dealing with the unbalance load is introduced, which has not been reported in the literature previously.

The above contributions pave the way for general analysis toward reluctance machines with unbalance windings and unbalance loads. 


\section{CHAPTER \\ TWO}

\section{SYSTEM MODELING}

The main concern of this research is the nonlinear characteristics of reluctance electric machine and its unbalance load operation. To illustrate the effectiveness of the proposed approach, a single-phase self-excited reluctance generator (SPSERG) is investigated. A mathematical model is indispensable for fully understanding this machine. In this chapter, suitable math models are developed to fit different analysis requirement.

The operation of SPSERG is load dependent. Therefore, this research will consider both a resistive load and a combination of a resistive and an inductive loads. The first load condition of the SPSERG is shown in Figure 1-1. This simple resistive load condition will be used in the initial analysis. After some understanding of the SPSERG has been obtained, a more general RL load will be investigated.

To fully understand the dynamic and steady state performance, mathematical model should be built first.

\subsection{System Model under ABC Natural Frame for Resistive Load}


In general, there are two approaches for electric machine modeling. One is the model in the natural frame and also called $A B C$ model. The other is in the reference frame, called QD model. The first model has the following advantages of using machine variables, and easy to compare with the actual measurement. But the problem is that the inductances are time variant and the model is hard to use to explain the steady state performance. This is the main reason why QD model is widely used in machine analysis. The QD model cannot only solve the transient response, but can also be applied for steady state analysis.

In Figure 1-1, the following electrical equations can describe the system with resistive load.

$$
\begin{aligned}
& V_{m}=-r_{m} I_{m}+\frac{d}{d t} \lambda_{m} \\
& V_{a}=-r_{a} I_{a}+\frac{d}{d t} \lambda_{a} \\
& I_{m}=-\frac{V_{m}}{R_{L}}-C_{m} \frac{d}{d t} V_{m} \\
& I_{a}=-C_{a} \frac{d}{d t} V_{a}
\end{aligned}
$$

where

$I_{m}:$ Current in main winding

$I_{a}:$ Current in auxiliary winding

$V_{m}:$ Terminal voltage of main winding

$V_{a}:$ Terminal voltage of auxiliary winding

$\lambda_{m}:$ Flux in main winding 


$$
\begin{aligned}
& \lambda_{a}: \text { Flux in auxiliary winding } \\
& C_{m}: \text { Terminal capacitance of main winding } \\
& C_{a}: \text { Terminal capacitance of auxiliary winding }
\end{aligned}
$$

The relationship between fluxes and currents is expressed by:

$$
\left[\begin{array}{l}
\lambda_{m} \\
\lambda_{a}
\end{array}\right]=\left[\begin{array}{cc}
L^{\prime}+L^{\prime \prime} \cos \left(2 \theta_{r}\right) & L^{\prime \prime} \sin \left(2 \theta_{r}\right) \\
L^{\prime \prime} \sin \left(2 \theta_{r}\right) & L^{\prime}-L^{\prime \prime} \cos \left(2 \theta_{r}\right)
\end{array}\right]\left[\begin{array}{c}
I_{m} \\
I_{a}
\end{array}\right]
$$

where

$\theta_{r}:$ Rotor angular displacement

The inductance matrix can also be attained by finite element method or measurement.

The saturation effect can be expressed by two nonlinear inductances named direct and quadratic inductances, as formulated in the following.

$$
\begin{aligned}
& L_{m d}=L^{\prime}+L^{\prime \prime}=f_{1}\left(\lambda_{\text {airgap }}\right) \\
& L_{m q}=L^{\prime}-L^{\prime \prime}=f_{2}\left(\lambda_{\text {airgap }}\right)
\end{aligned}
$$

So finally, equation (2-1) to equation (2-7) are used to solve the transient response. The saturation curves can be obtained by measurement or by finite element method.

\subsection{System Model under QD Reference Frame for Resistive Load}

The voltage equations for stator model are expressed as

$$
V_{m}=-r_{m} I_{m}+\frac{d}{d t} \lambda_{m}
$$




$$
V_{a}=-r_{a} I_{a}+\frac{d}{d t} \lambda_{a}
$$

The mechanical equation is described as:

$$
J \frac{d}{d t} \omega_{r}=T_{s}-T_{e}-\beta \omega_{r}
$$

In order to eliminate the time variant component in the inductance matrix, a rotor QD reference transform is used to simplify the model. The transform matrix is:

$$
K=\left[\begin{array}{cc}
\cos \theta_{r} & \sin \theta_{r} \\
\sin \theta_{r} & -\cos \theta_{r}
\end{array}\right]
$$

The inverse of this matrix is:

$$
K^{-1}=\left[\begin{array}{cc}
\cos \theta_{r} & \sin \theta_{r} \\
\sin \theta_{r} & -\cos \theta_{r}
\end{array}\right]
$$

Therefore, a change of the variables which formulates a transformation from one stationary two phase system to another rotor reference frame can be defined as:

$$
\left[\begin{array}{l}
f_{q} \\
f_{d}
\end{array}\right]=K\left[\begin{array}{l}
f_{m} \\
f_{a}
\end{array}\right]
$$

where $f$ can represent voltage, current or flux linkage.

Although the transformation to the reference frame is a change of variables and needs no physical meaning, it is often convenient to visualize the transformation equations as trigonometric relationships between variables shown in Figure 2-1. In particular, the equations of transformation may be thought of as if the $f_{q s}$ and $f_{d s}$ variables are directed along paths orthogonal to each other and rotating at an angular velocity of $\omega_{r}$. 


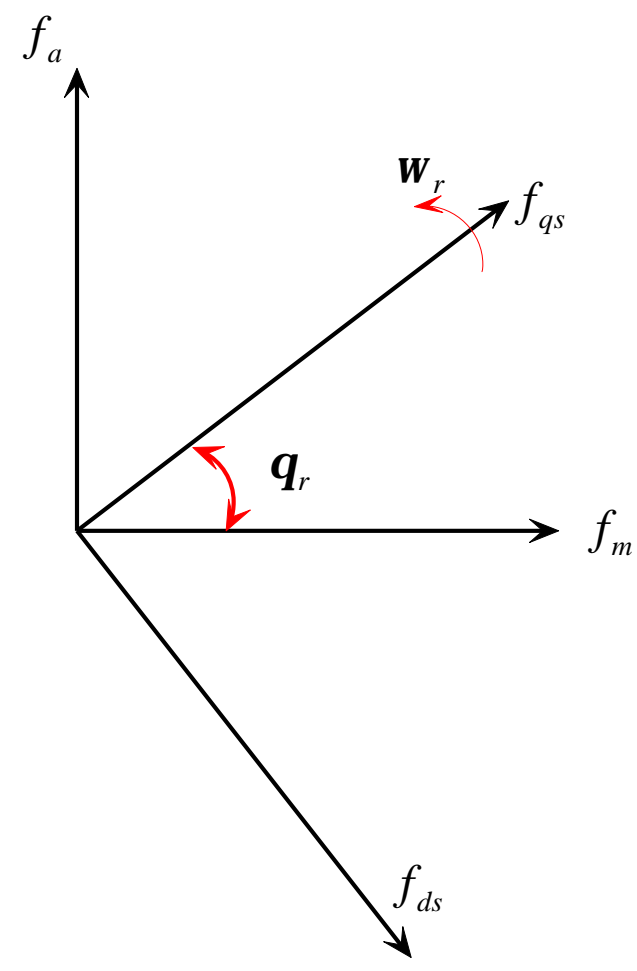

Figure 2-1: Transformation for two phase stator system to another two phase system The resultant QD model is:

$$
\begin{aligned}
& V_{q s}=-r_{q} I_{q s}+\omega_{r} \lambda_{d s}+\frac{d}{d t} \lambda_{q s} \\
& V_{d s}=-r_{d} I_{d s}-\omega_{r} \lambda_{q s}+\frac{d}{d t} \lambda_{d s}
\end{aligned}
$$

where

$$
\begin{aligned}
& \lambda_{q s}=L_{q s} I_{q s} \\
& L_{q s}=L_{m q}+L_{l q s} \\
& \lambda_{d s}=L_{d s} I_{d s} \\
& L_{d s}=L_{m d}+L_{l d s}
\end{aligned}
$$

$L_{m d}$ and $L_{m q}$ are nonlinear parameters obtained by measurement or FEM. 
When $C_{m}$ is connected in parallel with load and $C_{a}$ is connected in parallel with auxiliary winding, two terminal equations can be written as:

$$
\left.\left[\begin{array}{l}
I_{a} \\
I_{m}
\end{array}\right]=\left(\begin{array}{cc}
0 & 0 \\
0 & \frac{1}{R_{L}}
\end{array}\right)-\begin{array}{c}
V_{a} \\
V_{m}
\end{array}\right]+\frac{d}{d t}\left[\begin{array}{c}
Q_{a} \\
Q_{m}
\end{array}\right]
$$

Where

$Q_{a}:$ Charge in $C_{a}$

$Q_{m}:$ Charge in $C_{m}$

And

$$
\left[\begin{array}{l}
Q_{a} \\
Q_{m}
\end{array}\right]=\left[\begin{array}{cc}
C_{a} & 0 \\
0 & C_{m}
\end{array}\right]\left[\begin{array}{l}
V_{a} \\
V_{m}
\end{array}\right]
$$

So applying the transformation, we have:

$$
\left[\begin{array}{c}
I_{q s} \\
I_{d s}
\end{array}\right]=-K\left(\begin{array}{cc}
0 & 0 \\
0 & \frac{1}{R_{L}}
\end{array}\right) K^{-1}\left[\begin{array}{c}
V_{q s} \\
V_{d s}
\end{array}\right]-K \frac{d}{d t}\left(K^{-1}\left[\begin{array}{c}
Q_{q s} \\
Q_{d s}
\end{array}\right]\right)
$$

Expending the above matrix equation as:

$$
\begin{aligned}
& \frac{d}{d t} Q_{q s}+\omega_{r} Q_{d s}+\frac{1}{2 R_{L}}\left(1-\cos \left(2 \theta_{r}\right)\right) V_{q s}-\frac{1}{2 R_{L}} \sin \left(2 \theta_{r}\right) V_{d s}+I_{q s}=0 \\
& \frac{d}{d t} Q_{d s}-\omega_{r} Q_{q s}-\frac{1}{2 R_{L}} \sin \left(2 \theta_{r}\right) V_{q s}+\frac{1}{2 R_{L}}\left(\cos \left(2 \theta_{r}\right)+1\right) V_{d s}+I_{d s}=0
\end{aligned}
$$

Where

$$
\left[\begin{array}{c}
Q_{q s} \\
Q_{d s}
\end{array}\right]=\left[\begin{array}{cc}
\frac{C_{a}+C_{m}}{2}+\frac{C_{a}-C_{m}}{2} \cos 2 \theta_{r} & \frac{C_{a}-C_{m}}{2} \sin 2 \theta_{r} \\
\frac{C_{a}-C_{m}}{2} \sin 2 \theta_{r} & \frac{C_{a}+C_{m}}{2}-\frac{C_{a}-C_{m}}{2} \cos 2 \theta_{r}
\end{array}\right]\left[\begin{array}{c}
V_{q s} \\
V_{d s}
\end{array}\right]
$$

Using equations (2-14) though (2-21), system transient response can be analyzed. 
For the QD model, the time variant inductances are removed, however, the original time invariant load resistance and capacitances are now position or time dependent parameters. Therefore, for transient analysis, this model cannot offer any advantage. On the contrary, $\mathrm{ABC}$ model can solve the model with direct coupling of the machine variables, which can be easily integrated with the FEM model.

For steady state analysis, QD model is used. Because, in QD model, all inductances are constant and existing mature methods can be referenced. Moreover, basing on harmonic balanced technique, a revised QD model can be established to obtain the steady state solution.

\subsection{System Model under ABC Natural frame for RL Load}

The above model is for resistive load, which is simple and good for initial analysis and validation of the proposed method. But in practice, other kinds of load may be connected to the generator, such as single-phase induction motor. Therefore, to make the later proposed method applicable in general condition, an extended general model with RL load will be examined. 


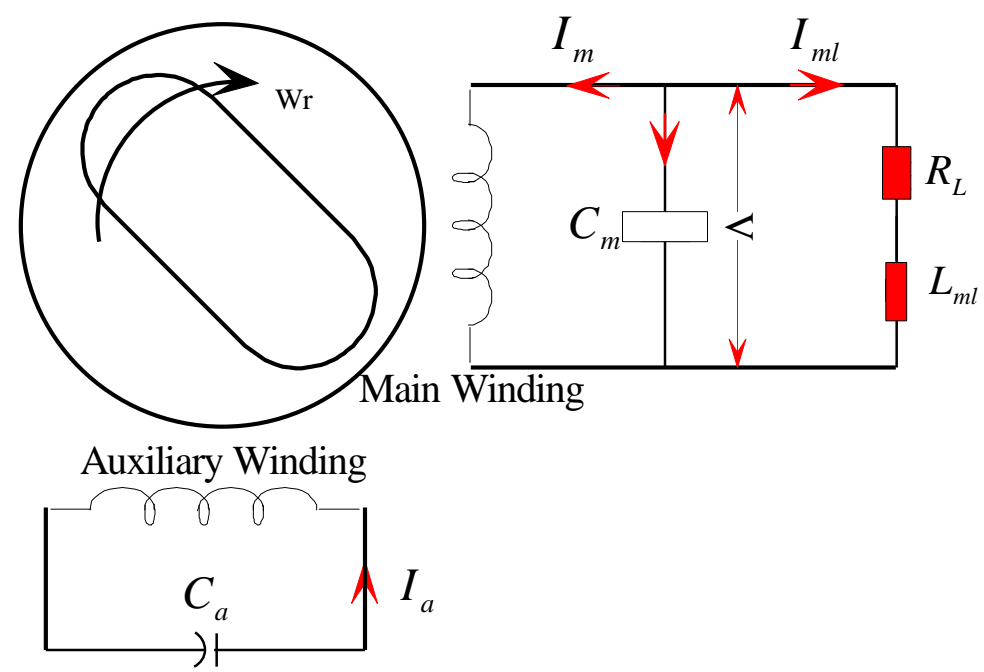

Figure 2-2: General schematic of the generator

As illustrated in Figure 2-2, the load connected to the main winding has resistance and inductance in series. The corresponding equations for RL type of load are:

$$
\begin{aligned}
& V_{m}=-r_{m} I_{m}+\frac{d}{d t} \lambda_{m} \\
& V_{a}=-r_{a} I_{a}+\frac{d}{d t} \lambda_{a} \\
& I_{m}=-I_{m l}-C_{m} \frac{d}{d t} V_{m} \\
& I_{a}=-C_{a} \frac{d}{d t} V_{a} \\
& V_{m}=R_{L} I_{m l}+L_{m} \frac{d}{d t} I_{m l}
\end{aligned}
$$

where

$$
\begin{aligned}
& I_{m l}: \text { Load current } \\
& L_{m}: \text { Load inductance }
\end{aligned}
$$

And all other variables and parameters are the same as described in Section 2.1. 


\subsection{System Model under OD Reference Frame for RL Load}

The stator equations in QD reference frame are exactly the same as equations (214) and (2-15) shown in Section 2.2.

However, it is not easy to transfer the terminal equations to QD reference frame. The problem is that in the load path, there is inductance, which needs a differential equation to describe. But there is no corresponding circuit in the auxiliary winding. From the reference frame theory, with a proper mathematical transformation, one system can be transferred into a new QD reference frame, which can simplify the model. But the system must have at least two phases, that is to say, reference frame theory cannot be used for single circuit.

Therefore, to make the QD model for this RL load condition, a fictitious circuit with very high resistance and low inductance is connected in parallel with the auxiliary winding, as shown in the dotted box in Figure 2-3. The idea is to use this added circuit to make the QD model possible. In the meantime, because of the big resistance and small inductance in the fictitious circuit, it is equal to an open circuit condition, which makes this model identical to the original terminal connection shown in Figure 2-2. 


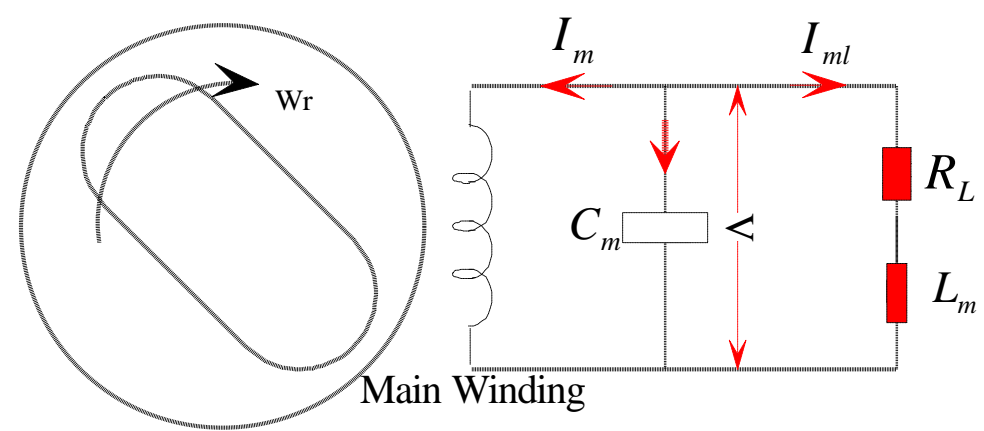

Auxiliary Winding

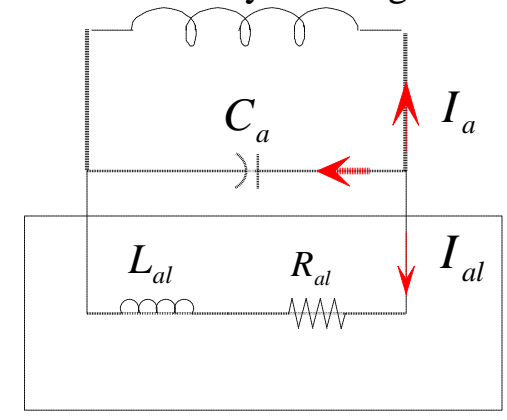

Figure 2-3: General schematic of the generator

Terminal equations can be expressed as:

$$
\begin{aligned}
& I_{m}=-I_{m l}-C_{m} \frac{d}{d t} V_{m} \\
& I_{a}=-I_{a l}-C_{a} \frac{d}{d t} V_{a} \\
& V_{m}=R_{L} I_{m l}+L_{m} \frac{d}{d t} I_{m l} \\
& V_{a}=R_{a l} I_{a l}+L_{a l} \frac{d}{d t} I_{a l}
\end{aligned}
$$

where

$I_{a l}:$ Current in added fictitious circuit

$L_{a l}$ : Inductance in added fictitious circuit

$R_{a l}:$ Resistance in added fictitious circuit 
Using the same transform as expressed in equation (2-13), equations (2-27) and (2-28) can be transferred to QD reference frame as:

$$
\begin{aligned}
& \frac{d}{d t} Q_{q s}+\omega_{r} Q_{d s}+I_{q l}+I_{q s}=0 \\
& \frac{d}{d t} Q_{d s}-\omega_{r} Q_{q s}+I_{d l}+I_{d s}=0
\end{aligned}
$$

where

$$
\left[\begin{array}{l}
Q_{q s} \\
Q_{d s}
\end{array}\right]=\left[\begin{array}{cc}
\frac{C_{m}+C_{a}}{2}+\frac{C_{m}-C_{a}}{2} \cos 2 \theta_{r} & \frac{C_{m}-C_{a}}{2} \sin 2 \theta_{r} \\
\frac{C_{m}-C_{a}}{2} \sin 2 \theta_{r} & \frac{C_{m}+C_{a}}{2}-\frac{C_{m}-C_{a}}{2} \cos 2 \theta_{r}
\end{array}\right]\left[\begin{array}{c}
V_{q s} \\
V_{d s}
\end{array}\right]
$$

However, for equations (2-29) and (2-30), if the same transform is used, then the equivalent resistance matrix is:

$$
R_{\text {equivalent }}=\begin{array}{cc}
\frac{R_{L}+R_{a l}}{2}+\frac{R_{L}-R_{a l}}{2} \cos 2 \theta_{r} & \frac{R_{L}-R_{a l}}{2} \sin 2 \theta_{r} \\
\frac{R_{L}-R_{a l}}{2} \sin 2 \theta_{r} & \frac{R_{L}+R_{a l}}{2}-\frac{R_{L}-R_{a l}}{2} \cos 2 \theta_{r}
\end{array}
$$

In order to simulate an open circuit condition, $R_{a l}$ has to have a large value. Therefore, $R_{L}$ has minimal effect in the model. To get rid of this problem, equations (229) and (2-30) have to be rewritten as:

$$
\begin{aligned}
& \frac{1}{R_{L}} V_{m}=I_{m l}+\frac{L_{m}}{R_{L}} \frac{d}{d t} I_{m l} \\
& \frac{1}{R_{a l}} V_{a}=I_{a l}+\frac{L_{a l}}{R_{a l}} \frac{d}{d t} I_{a l}
\end{aligned}
$$

Applying the transform equation (2-13), the resultant equations in QD reference frame are: 


$$
\begin{aligned}
& \frac{d}{d t} S_{q s}+\omega_{r} S_{d s}-\left(t_{1}+t_{2} \cos \left(2 \theta_{r}\right)\right) V_{q s}-t_{2} \sin \left(2 \theta_{r}\right) V_{d s}+I_{q l}=0 \\
& \frac{d}{d t} S_{d s}-\omega_{r} S_{q s}-t_{2} \sin \left(2 \theta_{r}\right) V_{q s}-\left(t_{1}-t_{2} \cos \left(2 \theta_{r}\right)\right) V_{d s}+I_{d l}=0
\end{aligned}
$$

where

$$
\left[\begin{array}{c}
S_{q s} \\
S_{d s}
\end{array}\right]=\left[\begin{array}{cc}
L_{1}+L_{2} \cos 2 \theta_{r} & L_{2} \sin 2 \theta_{r} \\
L_{2} \sin 2 \theta_{r} & L_{1}-L_{2} \cos 2 \theta_{r}
\end{array}\right]\left[\begin{array}{c}
I_{q l} \\
I_{d l}
\end{array}\right]
$$

And

$$
\begin{aligned}
& L_{1}=\frac{\frac{L_{m}}{R_{l}}+\frac{L_{a l}}{R_{a l}}}{2} \\
& L_{2}=\frac{\frac{L_{m}}{R_{l}}-\frac{L_{a l}}{R_{a l}}}{2} \\
& t_{1}=\frac{\frac{1}{R_{l}}+\frac{1}{R_{a l}}}{2} \\
& t_{2}=\frac{\frac{1}{R_{l}}-\frac{1}{R_{a l}}}{2}
\end{aligned}
$$

This QD model will be used in later steady state analysis.

From modeling point of view, the mathematical model for RL load represents a more general system description. If the inductance in the load circuit is small enough, then it can be a special case for resistive load. 


\section{CHAPTER \\ THREE}

\section{MAGNETIC MODEL}

\subsection{Introduction}

The finite element method (FEM) is a numerical technique for solving engineering field problems, which involves differential equations applied over regions constrained by boundary conditions. Even though the governing equations and boundary conditions for most problems can easily be determined, it is usually difficult or impossible to find a closed-form, analytic solution. The finite element method provides a way to obtain an accurate numerical solution for such problems by discretizing the model into small, interconnected elements and solving the governing equations for each small element. The elements are joined by ensuring the boundaries of each element compatible with those of its neighbors and with the overall boundary conditions of the model. After setting up the differential equations for the elements and applying the load and boundary conditions, the global matrix equation can be solved at the nodes.

When material property such as nonlinear magnetic permeability is involved, the Newton-Raphson method or other numerical techniques would be used to solve the matrix equations iteratively till a solution converges. 
In this study, the finite element method is used to solve the magnetic fields in the reluctance machine in a magnetostatic case.

The governing equations in magnetostatic analysis are the following subset of Maxwell's equations:

$$
\begin{aligned}
& \nabla \bullet \mathbf{B}=0 \\
& \nabla \times \mathbf{H}=\mathbf{J}
\end{aligned}
$$

where
B magnetic flux density vector
H magnetic field intensity vector
J total current density vector

The constitutive relation that describes the behavior of the magnetic material is given as:

$$
\mathbf{B}=\mu \mathbf{H}
$$

where

$$
\mu \quad \text { magnetic permeability matrix }
$$

For nonlinear magnetic materials, the magnetic permeability in the constitutive equation is given as a function of the field intensity, $\mathbf{H}$.

The magnetic vector potential, $\mathbf{A}$, can be defined as

$$
\mathbf{B}=\nabla \times \mathbf{A}
$$

The magnetic vector potential A from equation (3-4) along with the constitutive relation given in equation (3-3) can be substituted into equations (3-1) and (3-2) to obtain new equations in terms of the magnetic vector potential as

$$
\nabla \bullet \nabla \times \mathbf{A}=0
$$




$$
\nabla \times \nabla \times \mathbf{A}=\mu \mathbf{J}
$$

Furthermore, the Coulomb gauge can be defined as:

$$
\nabla \bullet \mathbf{A}=0
$$

With coupling of the Coulomb gauge to the vector identity $\nabla \times \nabla \times \mathbf{A}=\nabla(\nabla \cdot \mathbf{A})-\nabla^{2} \mathbf{A}$, equation (3-6) can be expressed in a more manageable form as:

$$
\nabla^{2} \mathbf{A}=-\mu \mathbf{J}
$$

In the two dimensional analysis used in this research, the model is defined in the 2-D polar plane, and the winding currents flow in only the z-direction. Therefore, the applied load, $\mathbf{J}$, has only a z-component as shown in the following expression.

$$
\mathbf{J}=J_{Z} \hat{\mathbf{a}}_{\mathbf{z}}
$$

Moreover, the magnetic vector potential A possesses the z-component only, and has the same vector form as the applied load.

$$
\mathbf{A}=A_{Z}(x, y) \hat{\mathbf{a}}_{\mathbf{z}}
$$

Taking equations 3-9 and 3-10 to equation 3-8, a scalar form can be obtained for this special case as:

$$
\frac{\partial^{2}}{\partial x^{2}} A_{Z}+\frac{\partial^{2}}{\partial y^{2}} A_{Z}=-\mu J_{Z}
$$

Equation 3-11 is the elemental differential equation, which must be solved for each element of the model. These elemental differential equations are assembled into a matrix equation representing the behavior of the whole system. Considering the appropriate boundary conditions, the field solution can be obtained. 


\subsection{FE Modeling for SPSERG}

Generally, there are six steps for the finite element analysis.

1): Build the geometry

2): Mesh the region

3): Assign materials

4): Assign load and boundary condition

5): Solve the field with proper solver

6): Post processing

For this application, a FEM package called ANSYS ${ }^{\circledR}$ is used. ANSYS is operated in the batch mode in such a way that a program file is read into the ANSYS processor and the output results are written to an output file. This method is in contrast to using the graphical user interface (GUI) to enter and analyze the model graphically.

It is necessary to use batch mode in this analysis because the state space routine that will be employed is in the MATLAB environment. Therefore, a method is needed to generate and analyze an ANSYS program from the MATLAB environment. To link the two packages together and validate the interactive process, the batch processor in ANSYS is the ideal solution.

In this study, MATLAB and ANSYS are joined in a master-slave manner. MATLAB will control the process and manage the jobs. ANSYS works as an external function, which can be called directly from MATLAB.

An ANSYS batch file would be created in a MATLAB routine, which bases on information about the machine geometry, stator coil currents and the rotor position. 
Before investigating the MATLAB routine, which generates the ANSYS batch file, it is instructive to examine the structure of the ANSYS batch program.

The ANSYS environment is divided into several subsections:

(1): /BEGIN section: define general parameters

(2): Preprocessor (/PREP7): develop the model of the magnetic system

(3): Solution processor (/SOLU): Obtain the finite element solutions

(4): Postprocessor (/POST1): generate the outputs

The ANSYS batch files generated in this study start with the /PREP7 preprocessor section. First, the element type is selected and its parameters are defined. For this analysis, the PLANE53 element is selected, which can model two-dimensional magnetic fields in either planar or axisymmetric configurations. The element is based on a magnetic vector potential formulation and is used for magnetostatic analysis.

In this case, the whole machine geometry is used, even though one could use half of the machine geometry in the FE mesh and computation to save the computation effort. The reason is that the anti-periodic condition and the unbalance two-phase winding layouts need to be accommodated for purposes of generality of the model. Moreover, one needs careful and close examination of the entire winding layout and the rotor position. Considering the whole machine geometry eliminates the possible mistake of misjudging the boundary conditions of the FEM model, and provides magnetic field solutions naturally reflecting the "anti-periodic" phenomenon.

Once the element type is specified, the properties for all of the materials used in the model are specified. In particular, the B-H curve for the electrical steel used for the 
stator and rotor is provided to ANSYS to integrate the nonlinearity. Then, the areas are defined by listing all of the key points. The order of the list is arranged by looping around the area in a counter clockwise fashion.

When listing the key points, which define an area, it is important to list all of the key points on the perimeter of the area, not just those at the vertexes of the particular area. If all of the key points on the perimeter are not included, the ANSYS processor will not recognize the interface between adjacent areas and will produce erroneous results. Figure 3-1 illustrates the geometry of the SERG.

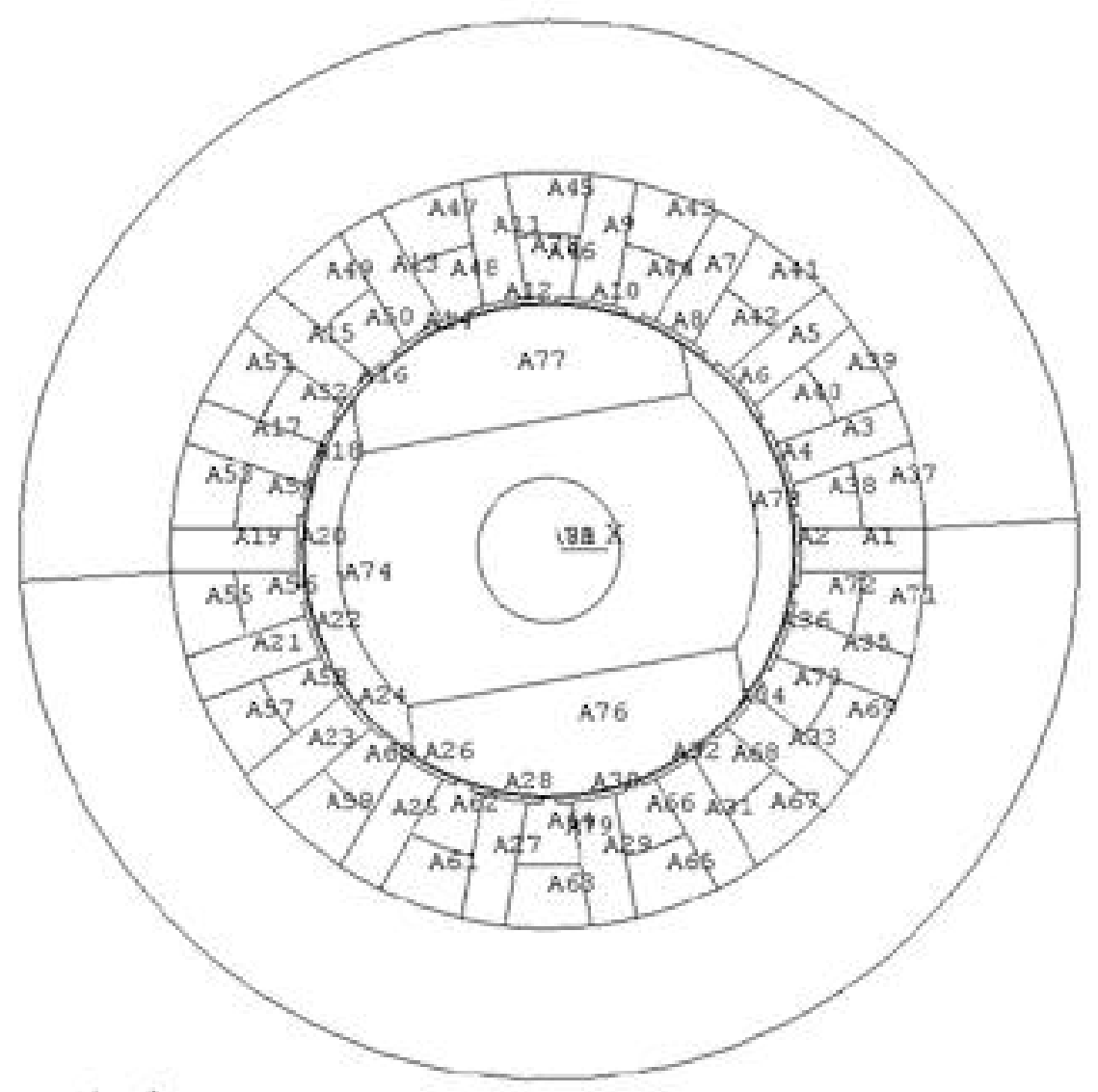

Figure 3-1 Geometry definition of the SPSERG 
The next step in the ANSYS program is to create names for each model area. Although this is not necessary for calculating the results, naming the areas makes the ANSYS program easier to write, read, and debug. Once the names are assigned, they are used to allocate the previously defined material numbers to the respective model areas.

After all areas are defined and assigned material properties, the model can now be meshed. Meshing of the model involves dividing each area into small elements, which will be used for the finite element solution. For this two-dimension analysis, the domain of interest can be discretized into a number of simple triangular or rectangular elements with homogenous properties. Using extremely flexible triangular elements, a very good approximation of the geometry is obtained.

The ANSYS processor provides an automated method for meshing the model in which the software chooses the meshing parameters. But, sometimes, this method may provide rough meshing in whole model. With command "smrtsize,n", the meshing can be refined by adjusting the degree parameter n. Figure 3-2 shows the meshing results.

After meshing, boundary conditions will then be applied to the model. Next, current density is employed to conducting areas as the excitation source.

Before solving the field, some preparation have to be done for obtaining the key parameters-inductances. LMATRIX is used to calculate an inductance matrix for an Nwinding system where $\mathrm{N}$ is the number of coils in the system. To invoke the LMATRIX macro, the elements for each coil must be grouped into a component. Each set of independent coil elements is assigned a component name with a prefix followed by the coil number. To invoke the LMATRIX macro, a vector array parameter with dimension 
$\mathrm{N}$ must be defined and named. Each entry of the vector array represents a unique coil.

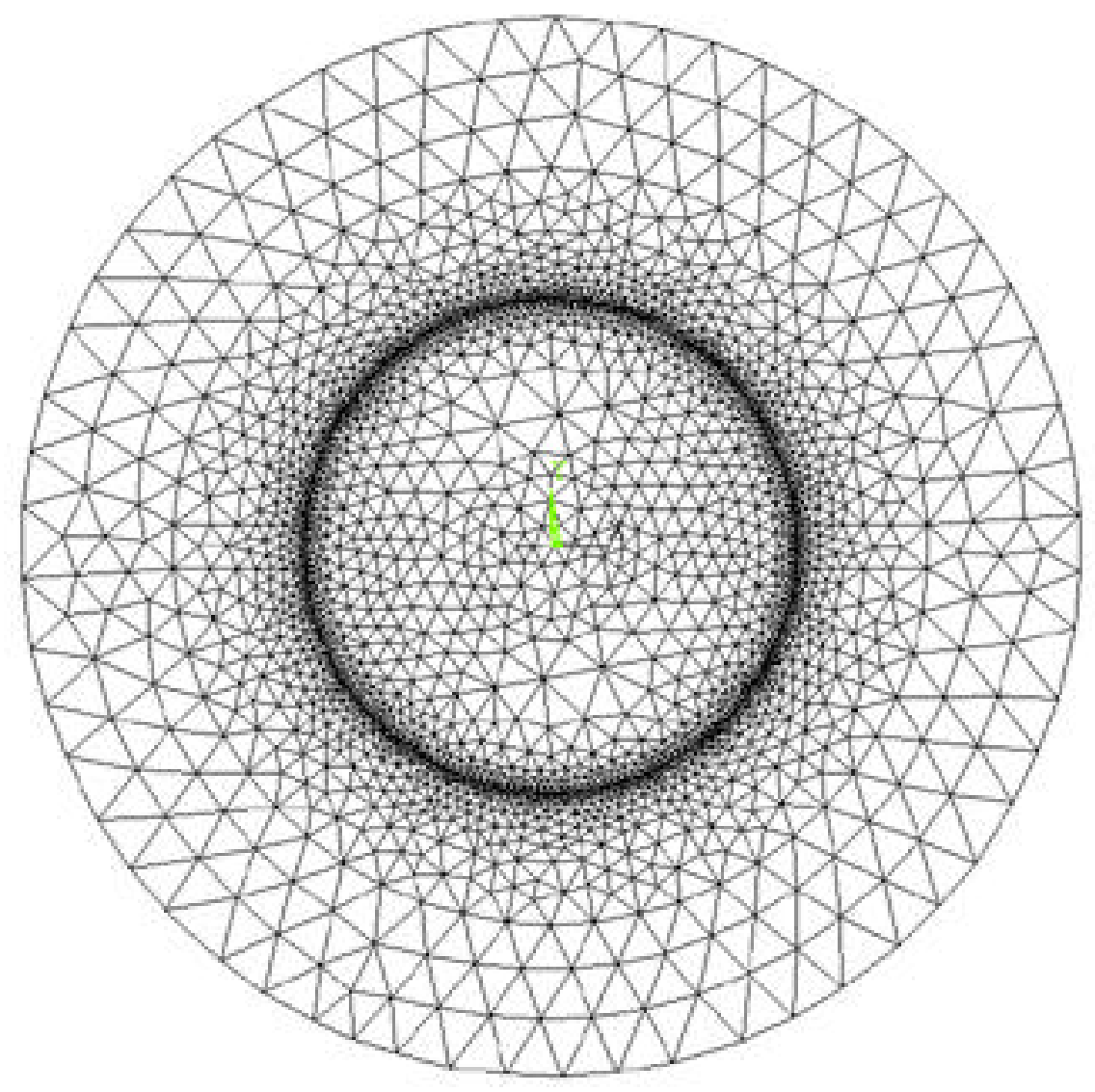

Figure 3-2: Meshing of the SPSERG

The vector array entries are set equal to the nominal current per turn in the coils at the operating point. Zero current values must be approximated by a negligibly small applied current.

At last, the ANSYS solution processor (/SOLU) is selected, and the solution is performed.

Once the solution is found, the postprocessor (/POST1) is utilized to examine the results. For this analysis, the inductance matrix is the most important result, which will be saved in the file and be extracted in later analysis. With the results exported to a file, the ANSYS batch file ends. 
Figure 3-3 displays the magnetic field solution under excitation condition $I_{m}=1 \mathrm{~A}$ and $I_{a}=6 \mathrm{~A}$.

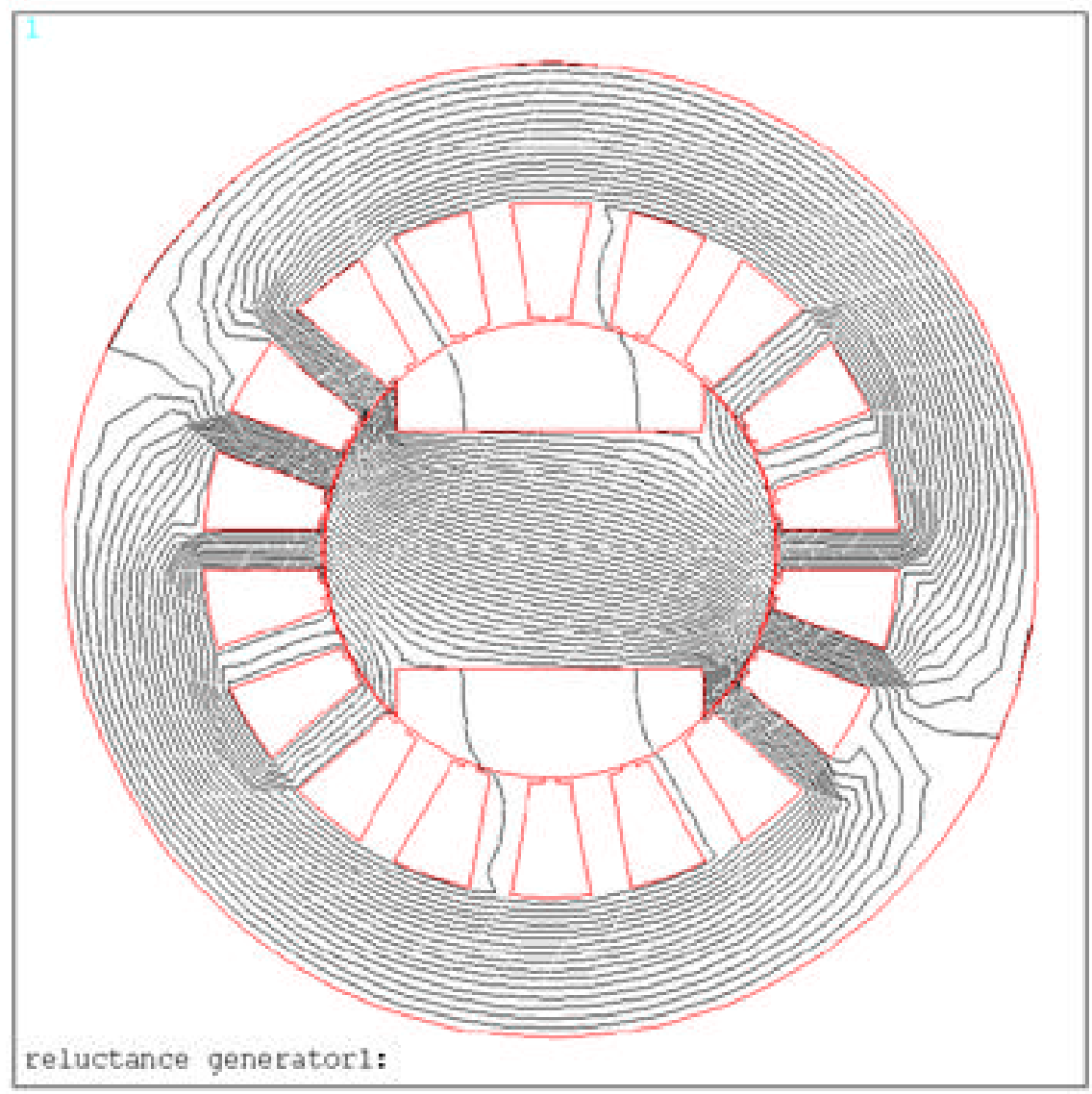

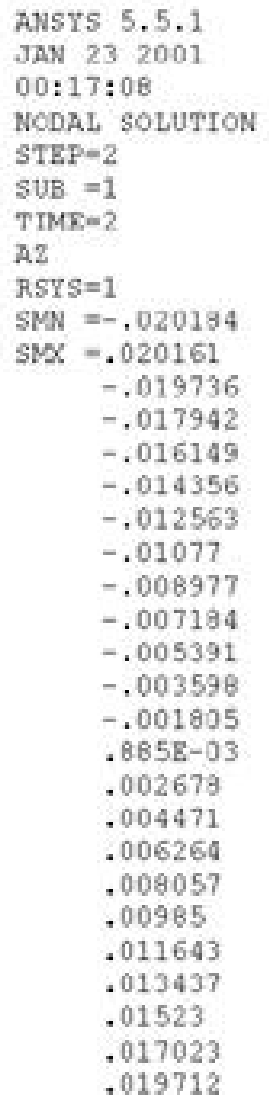

Figure 3-3: The magnetic field of the SPSERG

\subsection{Obtaining the Inductance}

To implement the off-line FE+SS method, the link between the two methods is the inductance matrix of the SPSERG.

The accurate calculation of machine winding inductances and their variation with saturation is crucial to the dynamic analysis. The energy perturbation method [24] is used. It is based on the perturbation of the magnetic field distribution inside the machine 
for a given set of winding currents (saturation level) by means of small current increments (perturbations). The magnetic field distribution and the energy perturbations due to such current increments are determined by means of the finite element method.

In ANSYS, the energy perturbation method is coded as an embedded macro named "Lmatrix" to compute the inductance matrix for multi-winding system.

Once the field solution is found, Lmatrix will be employed to obtain the inductance matrix of SPSERG.

Figure 3-4 shows the inductance profile under light load obtained from ANSYS, which equals to a linear condition.

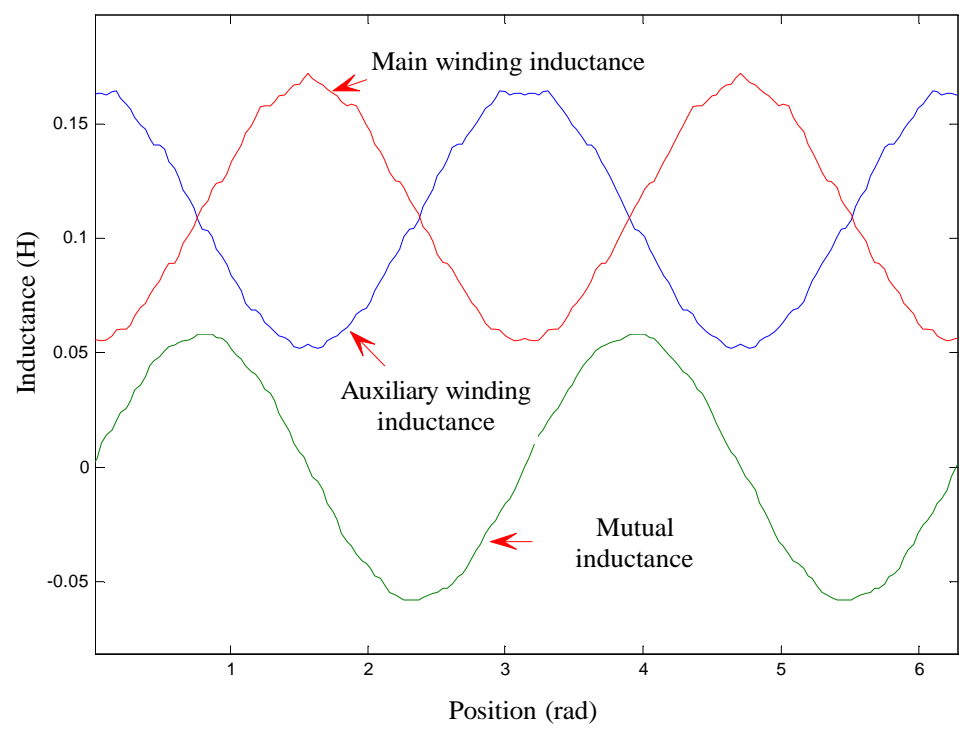

Figure 3-4: Inductance profile

The rotational step in the inductance computation is $2^{\circ}$. Since the inductances are not only load dependent but also position dependent, there is no general nonlinear instantaneous inductance profile. 


\section{CHAPTER FOUR}

\section{Transient Analysis Using Off- line FE+SS Method}

There are two possible approaches for machine performance analysis using state space model in conjunction with finite element method. One is to use the FEM to obtain the nonlinear characteristics off-line. The other is an on-line interactive process.

If computation effort is acceptable, point-by-point inductance update and an iterative process should be desirable, which means at every position, the inductance will be solved and then feeds to the state space model iteratively to finally determine the inductance and state variables. It can be called " on-line " or "time-stepping" inductance computation. The idea for this approach is to directly integrate the finite element method to the state space model. At every time step, the nonlinear inductance matrix is solved from the magnetic field, and feeds to system dynamic equations to update their parameters.

This method is very attractive, because the winding distribution is embedded in the field analysis, the harmonics of inductance can be directly represented. And the process can find the "actual" state variables under each distinct condition. However, in this work, the above ideal approach is limited by the computation capability of PC workstation, which is the platform for this analysis. 
In a design environment, designer wants to quickly know the rough performance for a given design without making any prototype. Hence, a simple numerical approach is always preferable. Even though the time stepping coupled state space model with finite element method can provide much higher accuracy, the computation effort is around several days in a PC workstation or several hours in a supercomputer, which is not necessary. Therefore, the off-line simple approach is chosen to predict the machine response and performance within reasonable accuracy, using very limited computation resource.

The key point of off-line method is how to obtain the nonlinear saturation characteristics of the machine. As described in Chapter 1 and Chapter 2, the saturation curve is essential for both transient and steady state analyses for the self-excited generator. Finite element method will be used to obtain these curves.

After the FEM is integrated in the system model, a complete numerical solution is established to make the design and performance evaluation in a totally computational environment, which will in turn shorten the design process and reduce the cost.

Similar to experimental process, currents are injected to the main and auxiliary windings in the finite element model. Then magnetic field is solved to obtain the flux distribution and at last the inductance matrix.

\section{$\underline{4.1}$ Measuring the Inductance}

Assume the space harmonics for winding distribution are neglected, so the problem is simplified as a balanced winding problem. The inductance measurement is by the following method. First, apply an AC voltage $(60 \mathrm{~Hz})$ to main winding, keep the 
auxiliary winding open circuited, rotor will naturally align with the stator which can be defined as initial position $\theta_{r}=0^{0}$. Then the current in main winding is measured. Secondly, move the rotor perpendicular to the initial position $\theta_{r}=90^{\circ}$. Apply the same voltage and measure the current. Thirdly, the direct and quadratic inductances can be computed base on the following average value equations (4-1) and (4-2).

$$
\begin{aligned}
L_{m d} & =\frac{V_{r m s}\left(0^{0}\right)}{2 \pi f I_{r m s}\left(0^{0}\right)} \\
L_{m q} & =\frac{V_{r m s}\left(90^{0}\right)}{2 \pi f I_{r m s}\left(90^{0}\right)}
\end{aligned}
$$

So the inductance matrix can be expressed as:

$$
L=\left[\begin{array}{cc}
\left(\frac{L_{m d}+L_{m q}}{2}\right)+\left(\frac{L_{m d}-L_{m q}}{2}\right) \cos 2 \theta_{r} & \left(\frac{L_{m d}-L_{m q}}{2}\right) \sin 2 \theta_{r} \\
\left(\frac{L_{m d}-L_{m q}}{2}\right) \sin 2 \theta_{r} & \left(\frac{L_{m d}+L_{m q}}{2}\right)-\left(\frac{L_{m d}-L_{m q}}{2}\right) \cos 2 \theta_{r}
\end{array}\right]
$$

Finally, the amplitude of the voltage is varied and corresponding currents for $\theta_{r}=0^{0}$ and $\theta_{r}=90^{\circ}$ are measured. At last, the relationship of the inductances vs current or flux can be plotted to show the nonlinear characteristics of the reluctance machine.

It is worth noting that because of the balance two windings, if the auxiliary winding is used to do the same measurement, the saturation curves are identical, which is verified by the actual measurement.

\subsection{Obtaining Inductance by FEM}

Mimicking the same process as the measurement, FEM is applied as the following. First, the rotor is located at the initial position which aligns to the main stator 
winding, directly assign a DC current to the main winding and a very small DC current to the auxiliary winding, which is required by the perturbation method. Secondly, solve the magnetostatic problem by the FEM. Then, the perturbation method is used to subtract the winding inductances. Thirdly, move the rotor $90^{\circ}$, and solve the problem by the same magnetostatic solver. So the direct and quadratic inductances are directly obtained and the same expression as shown in equations (4-1) and (4-2) can be reached.

However, one problem in this approach is that the inductances from FEM are the instantaneous values, as shown in Figure 4-1 and Figure 4-2. The relation between inductance and flux is not monotonous, which means, in high flux region, there are two inductances corresponding to one flux. This will make it hard to express the relationship analytically. Although the relation can be described by linear spline, it is still impossible to determine which inductance to be chosen by the same flux.

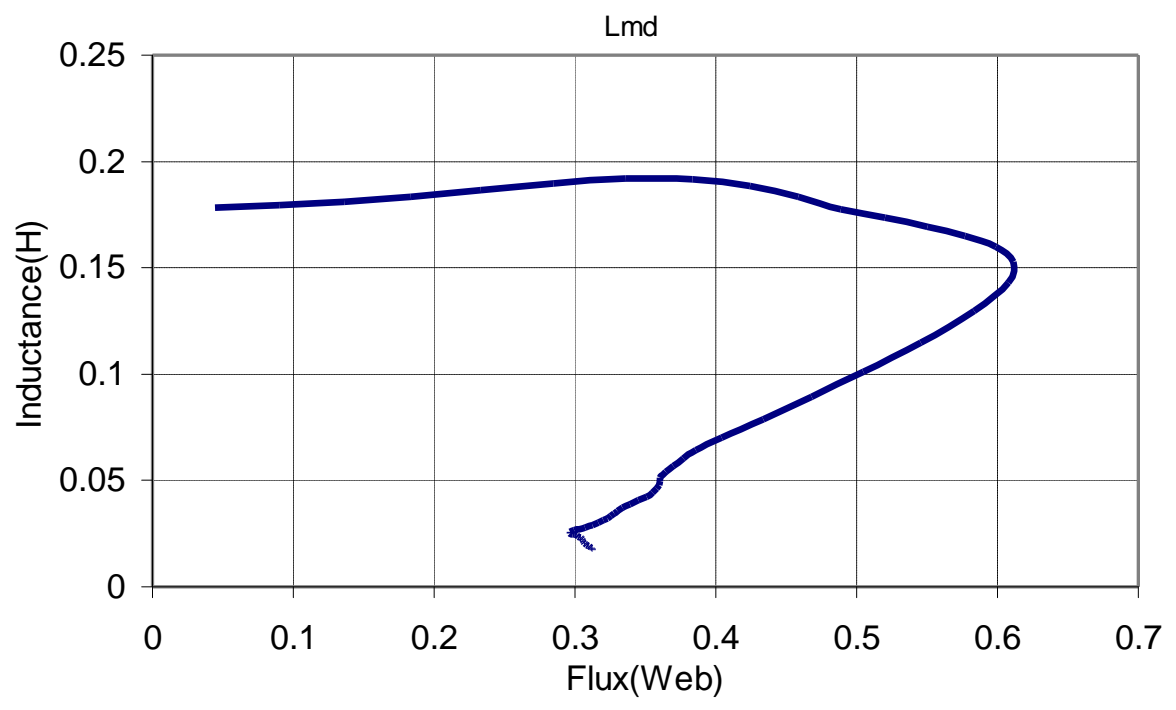

Figure 4-1: Instantaneous Lmd obtained from FEM 


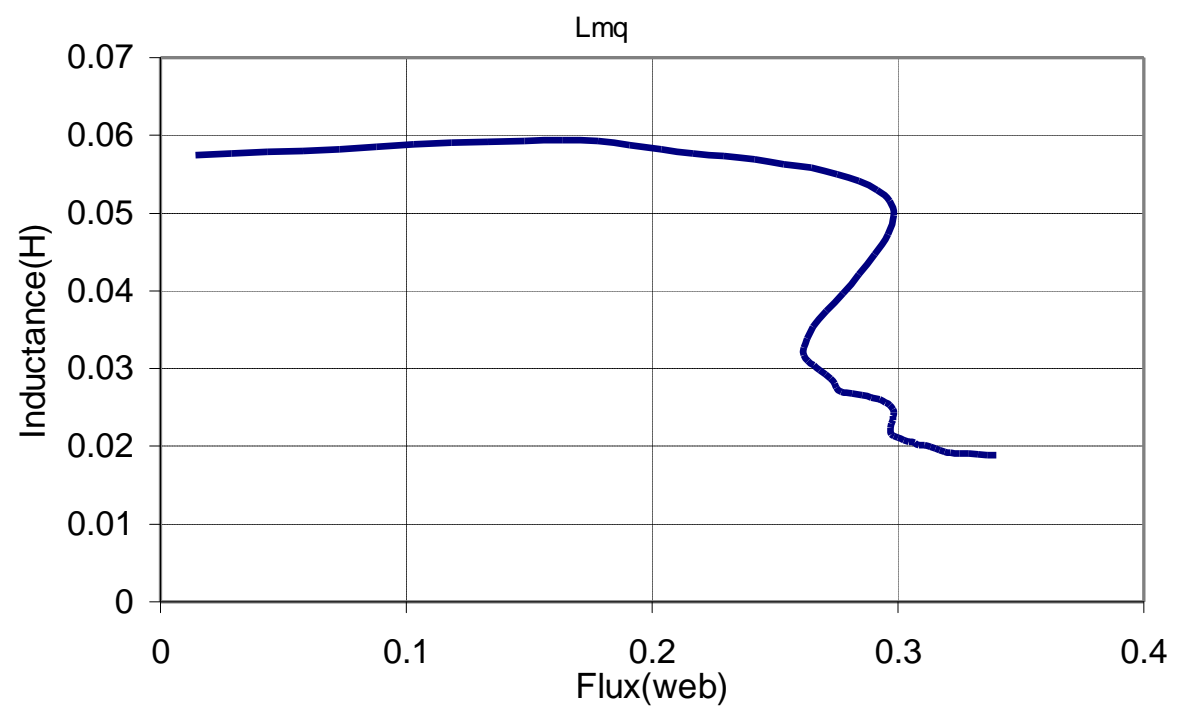

Figure 4-2: Instantaneous Lmq obtained from FEM

Even though it is possible to build the function $\lambda=f(L)$, it cannot be very helpful. Since in the simulation the flux is the obtained variables and the inductances are the resultant updated parameters. As a result, this is a one-way approach using a given flux to find a relevant inductance, but not vice versa.

So the key point is if the instantaneous inductance values are used, they must be integrated in the time stepping FEM+SS approach. Because there is no need to find the flux and the pertinent inductance, instead, currents are directly injected to the FEM model to obtain the inductance matrix, which avoid the problem described above.

However, because of the high computation effort by time stepping FEM+SS method, it is still a very useful and practical approach to find the saturation curve base on the FEM inductance results.

As we know, inductances from the measurement are average values. So an algorithm should be developed to transfer the FEM results to the average value representation. 


\subsection{Transformation}

It is more accurate to use time-stepping FEM+SS to simulate the system performance. But in reality, the computation effort is still very high compared to the state space plus measured saturation approach. So to contain the whole analysis in the simulation environment, which is desirable in principle design, the transform of the inductances obtained from FEM to equivalent inductances is a simple way to get the nonlinear characteristics.

In Figure 4-3, the inductances $L_{m d}$ and $L_{m q}$ vs current relationship by FEM are illustrated, basing on the approach suggested in Section 4.2.

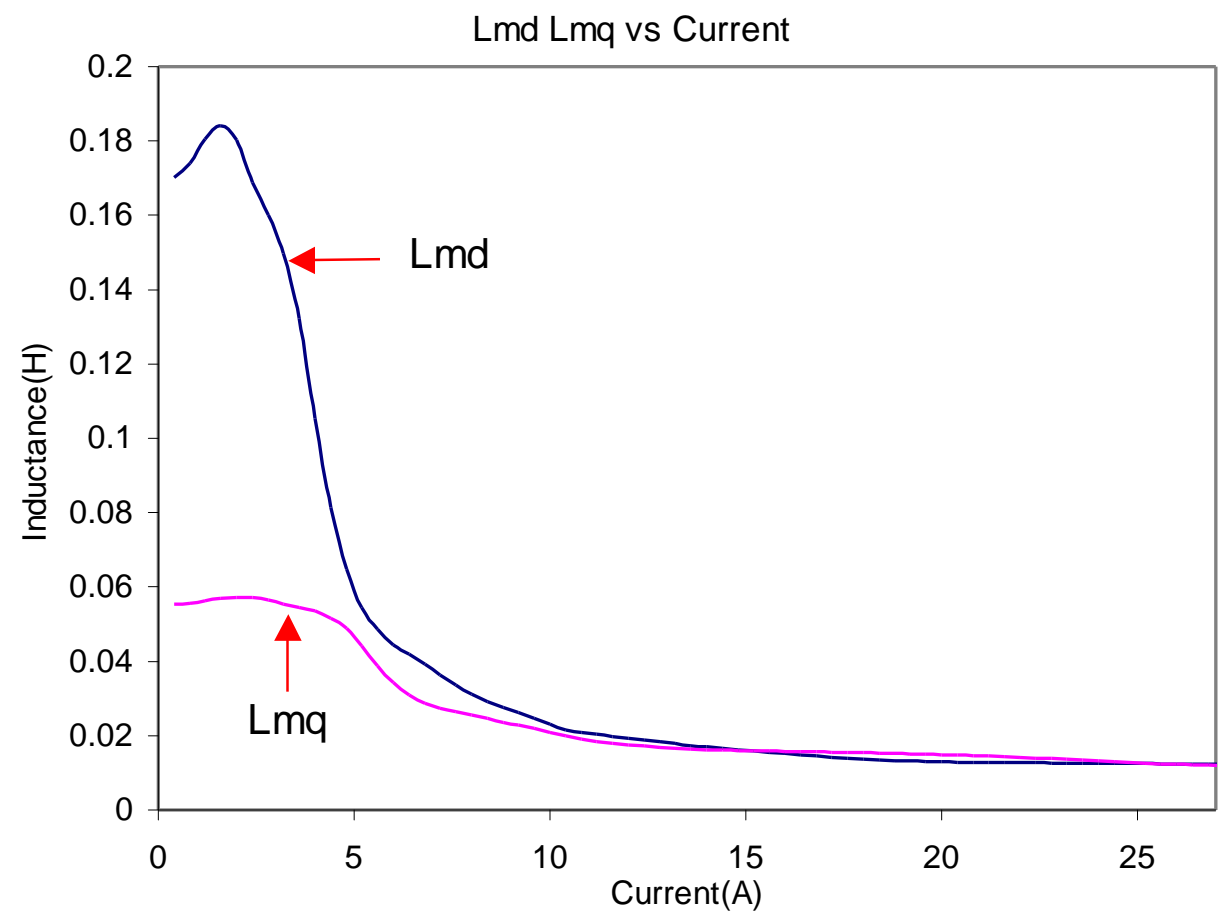

Figure 4-3: Current vs inductance relationship from FEM 
The analytic expressions for $L_{m d}$ and $L_{m q}$ are shown as :

$$
\begin{aligned}
L_{m d}= & -3.782 e-7 I_{m}^{6}+2.493 e-5 I_{m}^{5}-6.31 e-4 I_{m}^{4} \\
& +7.54 e-3 I_{m}^{3}-4.09 e-2 I_{m}^{2}+5.98 e-2 I_{m}+0.163 \\
L_{m q} & =-1.83 e-8 I_{m}^{6}+1.559 e-6 I_{m}^{5}-5.152 e-5 I_{m}^{4} \\
& +8.196 e-4 I_{m}^{3}-6.11 e-3 I_{m}^{2}+1.389 e-2 I_{m}+0.0478
\end{aligned}
$$

To find the average inductances, AC current is applied. Every instantaneous current in time domain can find corresponding inductances from Figure 4-3. The resultant inductance profiles and input AC current are plotted in Figure 4-4.

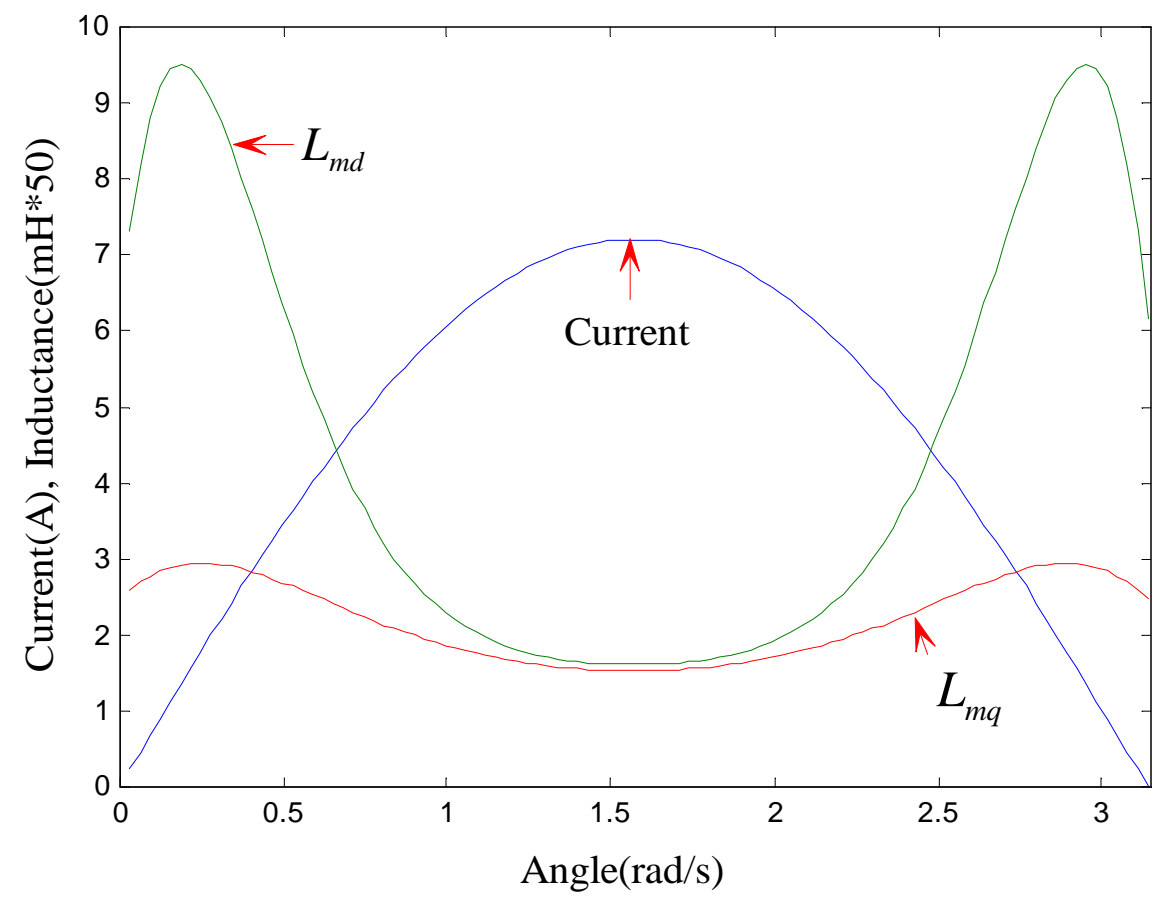

Figure 4-4: Applied AC current to main winding and the corresponding inductances

In Figure 4-4, to clearly illustrate the relation between current and inductances, the actual value is multiplied by a factor 50 to make it fit on the same plot with the 
current. It is clear that no matter how big is the $\mathrm{AC}$ current in the winding, only currents in the peak region cause the saturation. Therefore, to find the equivalent average inductance value as shown in equations (4-6) and (4-7), the following average value equations have to be applied.

$$
\begin{array}{r}
L_{\text {mdave }}=\frac{\int_{I(0)}^{I\left(\frac{\pi}{2}\right)} L_{m d}(I) d I}{I_{\max }-I_{\min }} \\
L_{\text {mqave }}=\frac{\int_{I(0)}^{I\left(\frac{\pi}{2}\right)} L_{m q}(I) d I}{I_{\max }-I_{\min }}
\end{array}
$$

And the corresponding fluxes are depicted as:

$$
\lambda=L_{\text {mdave }} I_{r m s}
$$

or

$$
\lambda=L_{\text {mqave }} I_{r m s}
$$

Analytic expression of the inductances vs flux can be directly established by data fitting and ready for the state space simulation. The computed saturation curves are shown in Figure 4-5. 


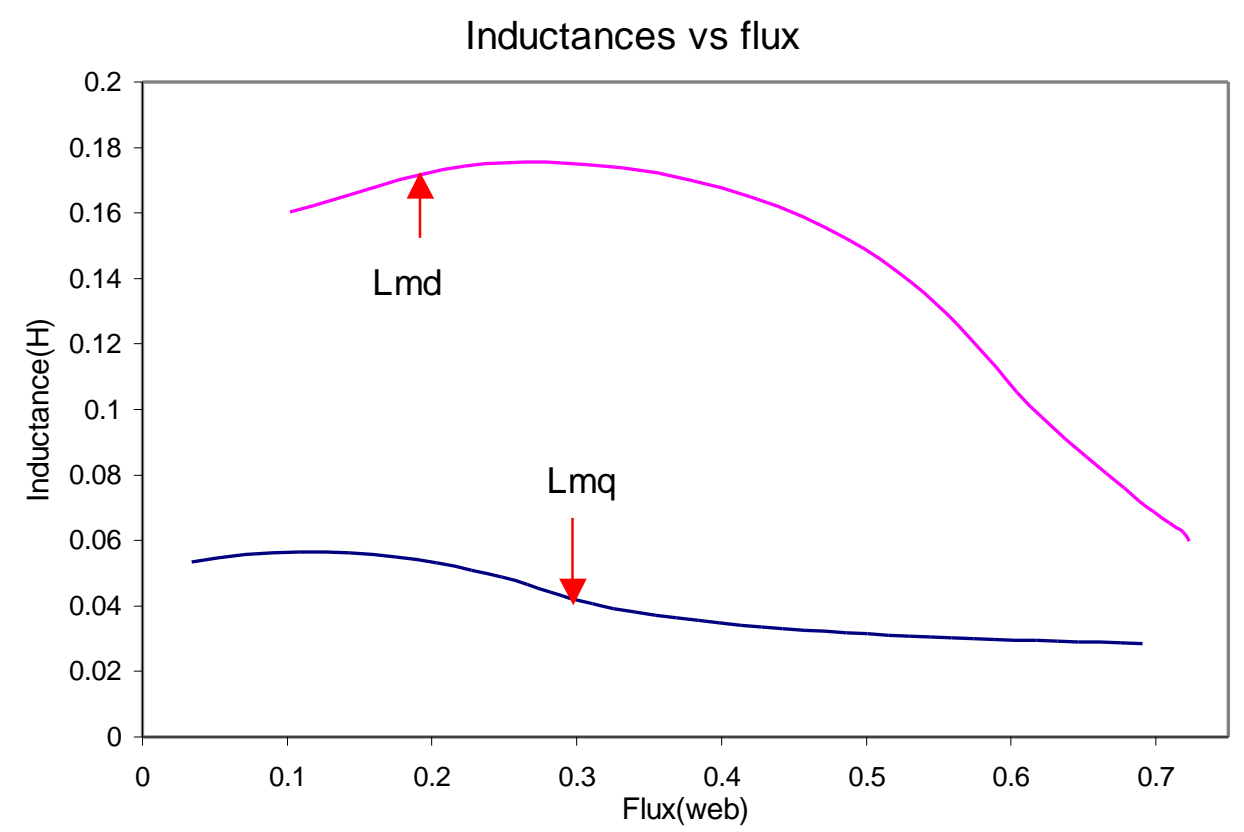

Figure 4-5: Flux vs inductance relationship from FEM

The analytic expressions for the inductance vs flux relationship are shown as :

$$
\begin{aligned}
L_{\text {mdave }}= & 12.23 \lambda^{5}-21.15 \lambda^{4}+13.06 \lambda^{3} \\
& -4.075 \lambda^{2}+0.674 \lambda+0.123 \\
L_{\text {mqave }}= & -266.49 \lambda^{6}+339.02 \lambda^{5}-162.23 \lambda^{4} \\
& +37.28 \lambda^{3}-4.77 \lambda^{2}+0.334 \lambda+0.044
\end{aligned}
$$

The whole process can be illustrated in the following flow chart Figure 4-6. 


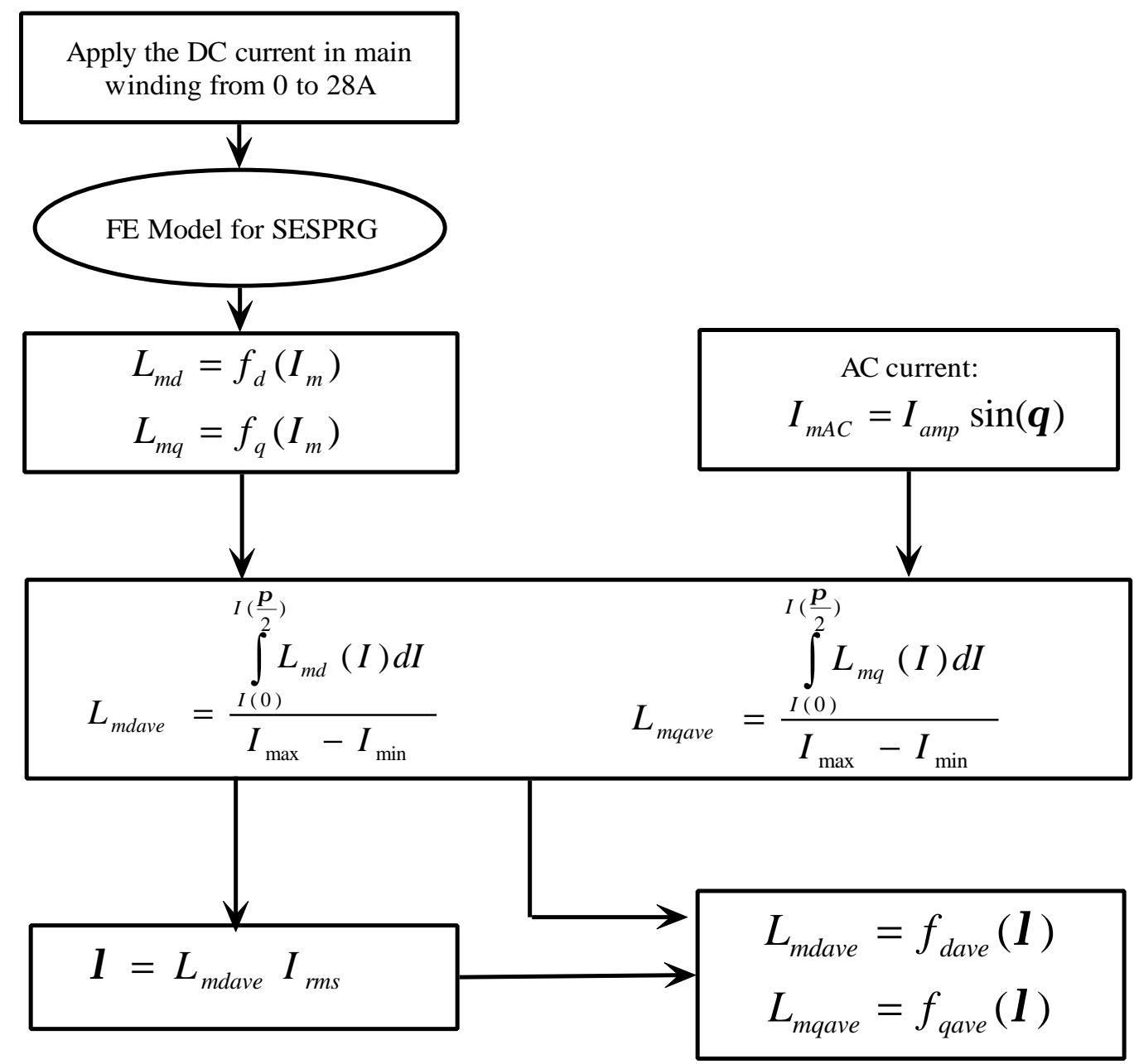

Figure 4-6: Flow chart for the process

After the computed saturation curves are obtained, the ABC model in Chapter 2 can be applied to simulate the transient response. 


\section{CHAPTER \\ FIVE}

\section{Steady State Analysis}

Even though results in transient analysis can also describe the wave form of state variables for steady state, from machine design and control points of view, parametric study is preferable to reveal the dependence of system parameters such as load impedance and terminal capacitance on self-excitation and output voltage. So a separate steady state analysis is necessary to depict not only the output waveform but also the parameter relationships.

Even though the qd model can get rid of the ripples caused by rotor saliency, because of the unbalanced loads of the reluctance generator, there are still ripples on the qd variables under steady state, which cannot be simply dealt with by qd model or average value method. In this research, the harmonic balance technique is employed to analyze the steady state performance.

\subsection{Harmonic Balance Technique}

Harmonic balance [25][26] can be seen as the extension of phasor analysis from linear to nonlinear differential equations. With harmonic balance, an approximate solution is found by assuming the solution to be a linear combination of sinusoids. Then 
the solution of the differential equation is substituted in and the resulting equation can be factored into a sum of purely sinusoidal terms. After that, superposition and the orthogonality of sinusoids at different harmonics can be exploited to break the resulting algebraic equation up to a collection of simpler equations, one for each harmonic. The equations are solved by finding the coefficients of the sinusoids in the assumed solution that result in the balancing of the algebraic equation at each harmonic.

This technique has been successfully applied in unbalance machine operations. Because it can be integrated with nonlinear characteristics, it is a suitable tool for this nonlinear analysis.

Algorithm of the harmonic balance:

Given: A differential equation of the form

$$
f(x, \dot{x}, u)=0
$$

where $u \in P\left(T_{0}\right)$ is the stimulus waveform, $x$ is the unknown waveform to be found and $f$ is contiuous and real.

Step 1: Assume that the solution $x$ exists, is real, and belong to $P\left(T_{0}\right)$.

Then

$$
x(t)=\sum_{k=-\infty}^{\infty} X(k) e^{j k \omega_{0} t} \quad \text { where } \omega_{0}=\frac{2 \pi}{T_{0}}
$$

Step 2: Substitute the assumed solution and its derivative into $f$. Note that $x \in P\left(T_{0}\right)$ implies $\dot{x} \in P\left(T_{0}\right)$, and since $u \in P\left(T_{0}\right)$ as well, $f(x, \dot{x}, u) \in P\left(T_{0}\right)$. Write the resulting equation as Fourier series: 


$$
f(x(t), \dot{x(t)}, u(t))=\sum_{k=-\infty}^{\infty} F(X, U, k) e^{j k \omega_{0} t}
$$

where

$$
\begin{aligned}
& X=[\ldots ., X(-1), X(0), X(1), \ldots .]^{T} \\
& U=[\ldots ., U(-1), U(0), U(1), \ldots . .]^{T} \\
& u(t)=\sum_{k=-\infty}^{\infty} U(k) e^{j k \omega_{0} t}
\end{aligned}
$$

Step 3: Solve the system of nonlinear algebraic equations

$$
F(X, U, k)=0 \text { for all } k \in \mathrm{Z} \text { for } \mathrm{X}
$$

\subsection{New Steady State Model Using Harmonic Balance Technique}

From transient simulation by abc model, the state variables can be transferred to qd model. It is observed that the qd variables are periodic and have double rotor frequency. Therefore, to apply harmonic balance in this problem, assume all the variables can be expressed in the form of:

$$
\begin{aligned}
& F_{q s}=F_{q s 1} \cos \left(\theta_{1}\right)+F_{q s 2} \sin \left(\theta_{1}\right)+F_{q s 3} \cos \left(\theta_{2}\right) \\
& F_{d s}=F_{d s 1} \cos \left(\theta_{1}\right)+F_{d s 2} \sin \left(\theta_{1}\right)+F_{d s 3} \cos \left(\theta_{2}\right)
\end{aligned}
$$

where:

$F$ can be voltage, current or flux.

$$
\theta_{1}=\theta_{e}+\theta_{r}=\left(\omega_{e}+\omega_{r}\right) t=2 \omega_{r} t \quad \theta_{2}=\theta_{e}-\theta_{r}=\left(\omega_{e}-\omega_{r}\right) t=0
$$


Actually, to obtain more accurate results, high order harmonics should be inclueded. However, in this case, the objective is to provide a general methodology. So only this simple form is used.

Another novel and important assumption is about the nolinear inductance. In this analysis, the saturation is assumed to lump to d-axis. The pertinent indutance is defined as:

$$
L_{d s}=L_{d s 1} \cos \left(\theta_{1}\right)+L_{d s 3}
$$

This assumption is based on the simulation results by abc model, as shown in Figure 5-1.

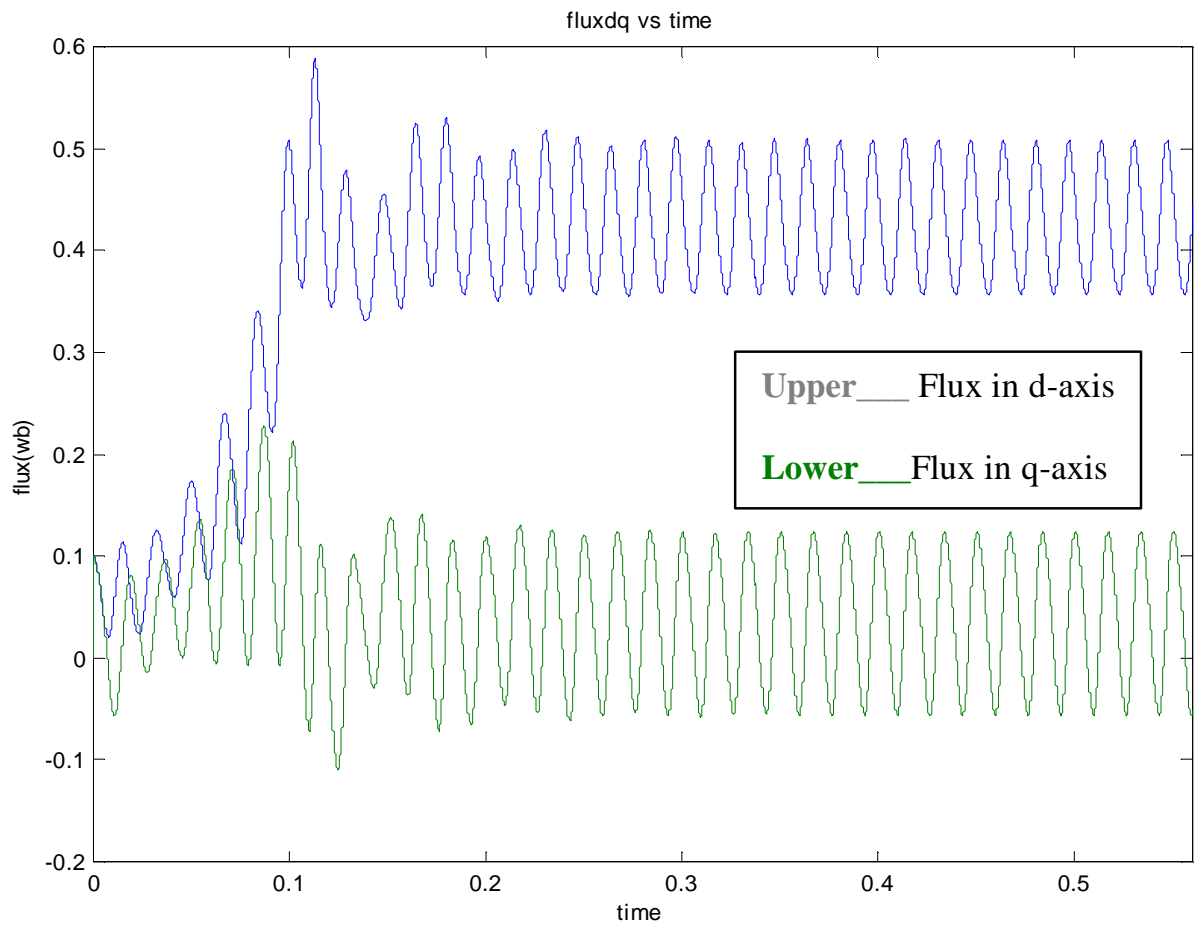

Figure 5-1: Flux in direct and quadratic axis vs time 
The d-axis flux is composed of a DC value and an AC component with double rotor frequency. Because of the monotonous relationship between inductance and flux, the relevant inductance should also have the same composition.

The relationship between fluxes and currents can be rewritten using harmonic balance technique as:

$$
\lambda=T I_{s}
$$

where

$$
T=\left[\begin{array}{cccccc}
L_{q s} & 0 & 0 & 0 & 0 & 0 \\
0 & L_{q s} & 0 & 0 & 0 & 0 \\
0 & 0 & L_{q s} & 0 & 0 & 0 \\
0 & 0 & 0 & L_{d s 3} & 0 & L_{d s 1} \\
0 & 0 & 0 & 0 & L_{d s 3} & 0 \\
0 & 0 & 0 & \frac{L_{d s 1}}{2} & 0 & L_{d s 3}
\end{array}\right]
$$

\subsubsection{Stator Model}

Then, stator equations (2-14) and (2-15)can be rewritten as 6 algebriac equations.

$$
\begin{aligned}
& V_{q s 1}=r_{a a} I_{q s 1}+\left(\omega_{e}+\omega_{r}\right) L_{q s} I_{q s 2}+\omega_{r} L_{d s 3} I_{d s 1}+\omega_{r} L_{d s 1} I_{d s 3} \\
& V_{q s 2}=-\left(\omega_{e}+\omega_{r}\right) L_{q s} I_{q s 1}+r_{a a} I_{q s 2}+\omega_{r} L_{d s 3} I_{d s 2} \\
& V_{q s 3}=r_{a a} I_{q s 3}+\omega_{r} L_{d s 3} I_{d s 3} \\
& V_{d s 1}=-\omega_{r} L_{q s} I_{q s 1}+r_{b b} I_{d s 1}+\left(\omega_{e}+\omega_{r}\right) L_{d s 3} I_{d s 2} \\
& V_{d s 2}=-\omega_{r} L_{q s} I_{q s 2}-\left(\omega_{e}+\omega_{r}\right) L_{d s 3} I_{d s 1}+r_{b b} I_{d s 2}-\left(\omega_{e}+\omega_{r}\right) L_{d s 1} I_{d s 3} \\
& V_{d s 3}=-\omega_{r} L_{q s} I_{q s 3}+r_{b b} I_{d s 3}
\end{aligned}
$$

In matrix form, we have 


$$
V_{s}=M I_{s}
$$

where

$$
M=\left[\begin{array}{cccccc}
r_{a a} & \left(\omega_{e}+\omega_{r}\right) L_{q s} & 0 & \omega_{r} L_{d s 3} & 0 & \omega_{r} L_{d s 1} \\
-\left(\omega_{e}+\omega_{r}\right) L_{q s} & r_{a a} & 0 & 0 & \omega_{r} L_{d s 3} & 0 \\
0 & 0 & r_{a a} & 0 & 0 & \omega_{r} L_{d s 3} \\
-\omega_{r} L_{q s} & 0 & 0 & r_{b b} & \left(\omega_{e}+\omega_{r}\right) L_{d s 3} & 0 \\
0 & -\omega_{r} L_{q s} & 0 & -\left(\omega_{e}+\omega_{r}\right) L_{d s 3} & r_{b b} & -\left(\omega_{e}+\omega_{r}\right) L_{d s 1} \\
0 & 0 & -\omega_{r} L_{q s} & 0 & 0 & r_{b b}
\end{array}\right]
$$

\subsubsection{Terminal Model with Resistive Load}

The terminal equations (2-19) and (2-20) can be expressed by harmonic technique

as:

$$
\begin{aligned}
& I_{q s 1}=\omega_{r} C_{1} V_{d s 1}+\left(\omega_{e}+\omega_{r}\right) C_{1} V_{q s 2}+\frac{V_{q s 1}}{2 R_{L}} \\
& I_{q s 2}=\omega_{r} C_{1} V_{d s 2}-\left(\omega_{e}+\omega_{r}\right) C V_{q s 1}+\frac{V_{q s 2}}{2 R_{L}} \\
& I_{q s 3}=\omega_{r} C_{1} V_{d s 3}+\frac{V_{q s 3}}{2 R_{L}}+\frac{1}{2} \omega_{r} C_{2}\left(V_{q s 2}-V_{d s 1}\right)+\frac{V_{q s 1}}{4 R_{L}}+\frac{V_{d s 2}}{4 R_{L}} \\
& I_{d s 1}=-\omega_{r} C_{1} V_{q s 1}+\left(\omega_{e}+\omega_{r}\right) C_{1} V_{d s 2}+\frac{V_{d s 1}}{2 R_{L}} \\
& I_{d s 2}=-\omega_{r} C_{1} V_{q s 2}-\left(\omega_{e}+\omega_{r}\right) C_{1} V_{d s 1}+\frac{V_{d s 2}}{2 R_{L}} \\
& I_{d s 3}=-\omega_{r} C_{1} V_{q s 3}+\frac{V_{d s 3}}{2 R_{L}}-\frac{1}{2} \omega_{r} C_{2}\left(V_{q s 1}+V_{d s 2}\right)+\frac{V_{q s 2}}{4 R_{L}}-\frac{V_{d s 1}}{4 R_{L}}
\end{aligned}
$$

Equations (5-13) to (5-18) can be expressed by matrix form: 


$$
I_{s}=-A V_{s}=N V_{s}
$$

where:

$$
A=\left[\begin{array}{cccccc}
\frac{1}{2 R_{L}} & 2 \omega_{r} C_{1} & 0 & \omega_{r} C_{1} & 0 & 0 \\
-2 \omega_{r} C_{1} & \frac{1}{2 R_{L}} & 0 & 0 & \omega_{r} C_{1} & 0 \\
-\frac{1}{4 R_{L}} & \frac{\omega_{r} C_{2}}{2} & \frac{1}{2 R_{L}} & -\frac{\omega_{r} C_{2}}{2} & -\frac{1}{4 R_{L}} & \omega_{r} C_{1} \\
-\omega_{r} C_{1} & 0 & 0 & \frac{1}{2 R_{L}} & 2 \omega_{r} C_{1} & 0 \\
0 & -\omega_{r} C_{1} & 0 & 2 \omega_{r} C_{1} & \frac{1}{2 R_{L}} & 0 \\
-\frac{\omega_{r} C_{2}}{2} & -\frac{1}{4 R_{L}} & -\omega_{r} C_{1} & \frac{1}{4 R_{L}} & -\frac{\omega_{r} C_{2}}{2} & \frac{1}{2 R_{L}}
\end{array}\right]
$$

\section{$\underline{\text { 5.2.3 Terminal Model with RL Load }}$}

Applying assumption (5-1) and (5-2) to the terminal equations (2-31) and (2-32),

we have the expression in matrix form.

$$
\left[\begin{array}{c}
I_{q s 1} \\
I_{q s 2} \\
I_{q s 3} \\
I_{d s 1} \\
I_{d s 2} \\
I_{d s 3}
\end{array}\right]=-\left[\begin{array}{cccccc}
0 & 0 & 0 & C_{1} \omega_{r} & 0 & 0 \\
0 & 0 & 0 & 0 & C_{1} \omega_{r} & 0 \\
0 & -\frac{C_{2} \omega_{r}}{2} & 0 & \frac{C_{2} \omega_{r}}{2} & 0 & C_{1} \omega_{r} \\
-C_{1} \omega_{r} & 0 & 0 & 0 & 0 & 0 \\
0 & -C_{1} \omega_{r} & 0 & 0 & 0 & 0 \\
\frac{C_{2} \omega_{r}}{2} & 0 & -C_{1} \omega_{r} & 0 & \frac{C_{2} \omega_{r}}{2} & 0
\end{array}\right]\left[\begin{array}{c}
V_{q s 1} \\
V_{q s 2} \\
V_{q s 3} \\
V_{d s 1} \\
V_{d s 2} \\
V_{d s 3}
\end{array}\right]-\left[\begin{array}{c}
I_{q l 1} \\
I_{q l 2} \\
I_{q l 3} \\
I_{d l 1} \\
I_{d l 2} \\
I_{d l 3}
\end{array}\right]
$$

Or describe as:

$$
I_{s}=-B V_{s}-I_{l}
$$

Using harmonic balance technique in equations 2-37 and 2-38, the resultant expression is: 


$$
\left[\begin{array}{cccccc}
t_{1} & 0 & 0 & 0 & 0 & 0 \\
0 & t_{1} & 0 & 0 & 0 & 0 \\
\frac{t_{2}}{2} & 0 & t_{1} & 0 & \frac{t_{2}}{2} & 0 \\
0 & 0 & 0 & t_{1} & 0 & 0 \\
0 & 0 & 0 & 0 & t_{1} & 0 \\
0 & \frac{t_{2}}{2} & 0 & -\frac{t_{2}}{2} & 0 & t_{1}
\end{array}\right]\left[\begin{array}{c}
V_{q s 1} \\
V_{q s 2} \\
V_{q s 3} \\
V_{d s 1} \\
V_{d s 2} \\
V_{d s 3}
\end{array}\right]=\left[\begin{array}{cccccc}
1 & 0 & 0 & L_{1} \omega_{r} & 0 & 0 \\
0 & 1 & 0 & 0 & L_{1} \omega_{r} & 0 \\
0 & -L_{2} \omega_{r} & 1 & \frac{L_{2} \omega_{r}}{2} & 0 & L_{1} \omega_{r} \\
-L_{1} \omega_{r} & 0 & 0 & 1 & 0 & 0 \\
0 & -L_{1} \omega_{r} & 0 & 0 & 1 & 0 \\
\frac{L_{2} \omega_{r}}{2} & 0 & -L_{1} \omega_{r} & 0 & \frac{L_{2} \omega_{r}}{2} & 1
\end{array}\right]\left[\begin{array}{c}
I_{q l 1} \\
I_{q l 2} \\
I_{q l 3} \\
I_{d l 1} \\
I_{d l 2} \\
I_{d l 3}
\end{array}\right]
$$

Or show as:

$$
C V_{s}=D I_{l}
$$

Substitute $I_{l}$ in equation 5-22, we have:

$$
I_{s}=-\left(B+C D^{-1}\right) V_{s}=N V_{s}
$$

\section{$\underline{5.3 \text { Proposed Algorithm }}$}

Equations (5-12) can be combined with equation (5-19) or equation (5-25) as:

$$
\left[M+N^{-1}\right] I=\bar{M} I=0
$$

This is a typical autonomous system. The solution of this system is either $I=0$, which is a trivial solution, or $|\bar{M}|=0$. So to find the parameter dependence for steady state, $|\bar{M}|=0$ has to be solved.

In steady state analysis for induction generator and three-phase reluctance generator, the air-gap flux is assumed first, then going back to QD model to solve for the machine variables. But in this unbalanced load case, the air-gap flux consists of not only a DC component but also an AC component which are determined by system parameters 
and cannot be assumed independently. So a new approach has to be found to start the analysis.

Due to the parameter coupling, the system performance is determined by seven parameters--load resistance $R_{L}$ and inductance $L_{m}$, rotor speed $\omega_{r}$, capacitors $C_{m}, C_{a}$ connected with main and auxiliary windings and the winding inductances $L_{q s}, L_{d s 1}, L_{d s 3}$. In steady state analysis, if any of the six in the above seven variables are given, the last variable can be solved to satisfy $|\bar{M}|=0$.

In this analysis, because of the self-excited nature of the system, the steady state analysis has to go backwards. A novel approach for steady state analysis, which can be called " inductance-oriented method", is proposed. The idea is that under certain condition, which can be defined by fixed capacitors and a given load resistance and inductance, solve the winding inductances first, then find the relevant fluxes and finally attain the machine variables. A simple schematic process is in Figure 5-2. So if the resistance is changed, steady state performance and parametric dependence can be described by the serial solutions.

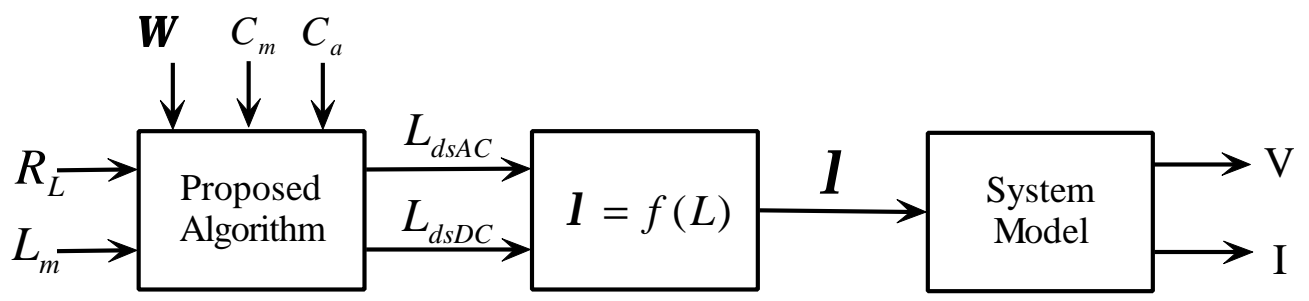

Figure 5-2: Schematic drawing for the steady state analysis

In details, the process is as following. Given a load resistance and inductance, rotor speed and excited capacitors, first, assuming the AC component in equation (5-3) to 
be zero, solve $|\vec{M}|=0$ to obtain the DC component $L_{d s 3}$. This equals to solve a balanced condition. Then taking $L_{d s 3}$ back to equation (5-26), the position dependent inductance $L_{d s 1}$ is obtained sustaining the same constraint $|\bar{M}|=0$.

With the established relationship between inductance and flux, which is obtained by measurement or finite element solution, the flux can be determined. Further variables such as output voltage and current are subsequently solved by equations (5-4) and (5-12).

The detailed description for the process is shown in Figure 5-3.

After the components in equations (5-4) and (5-12) are obtained, the harmonic balance technique can be used inversely to construct the waveforms for all the variables. Then the rms values for all variables can be computed by manipulating of the sinusoidal combinations, or by applying the discrete time series to construct the waveform and numerically obtain the rms value.

\subsection{Reconstruction of the State Variable's Waveform}

With the obtained coefficients of the qd variables, it is necessary to reconstruct the state variables to illustrate the variable's waveform and get their rms values. The process is in Figure 5-4. 


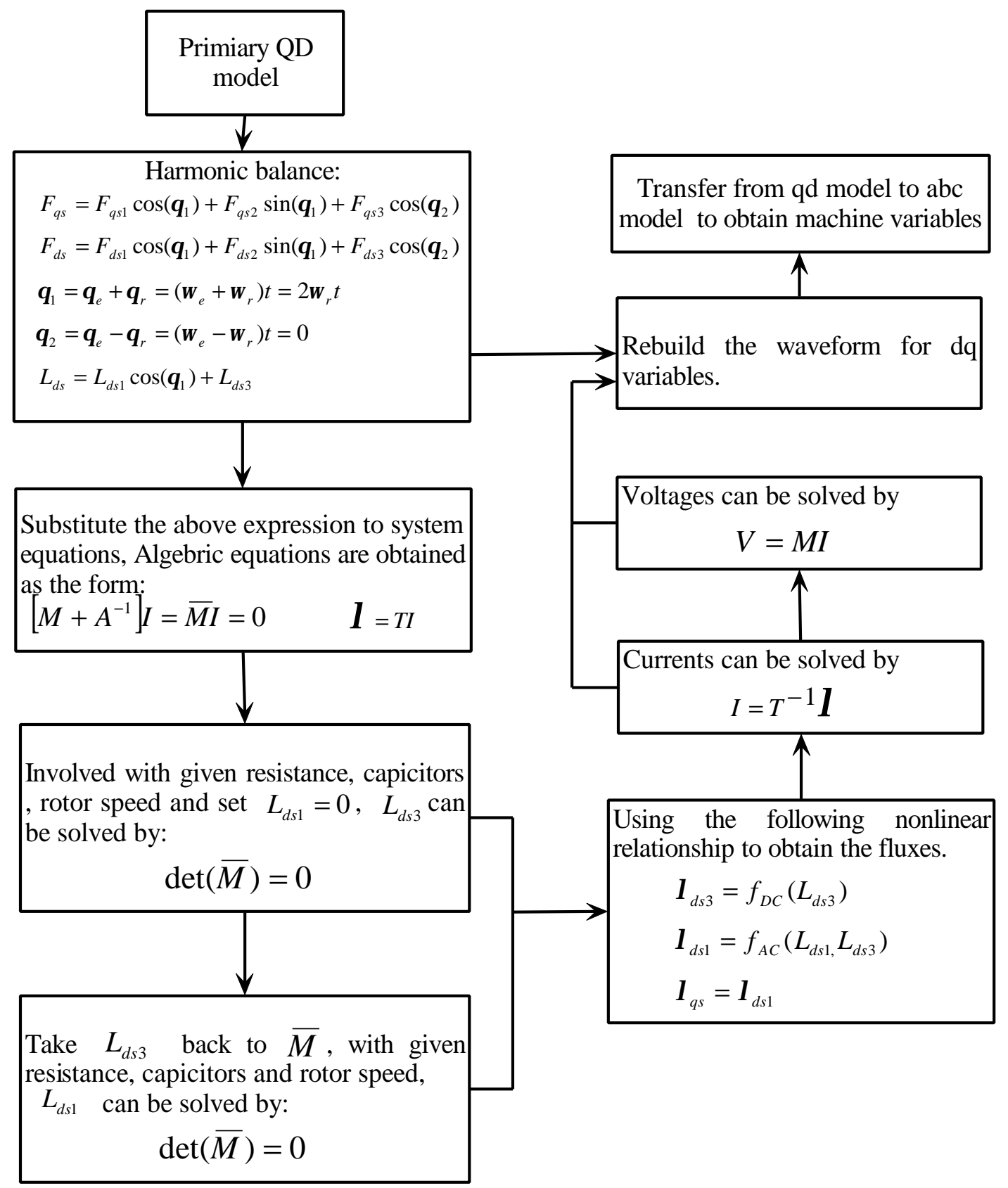

Figure 5-3: Flow chart for the novel steady state analysis 


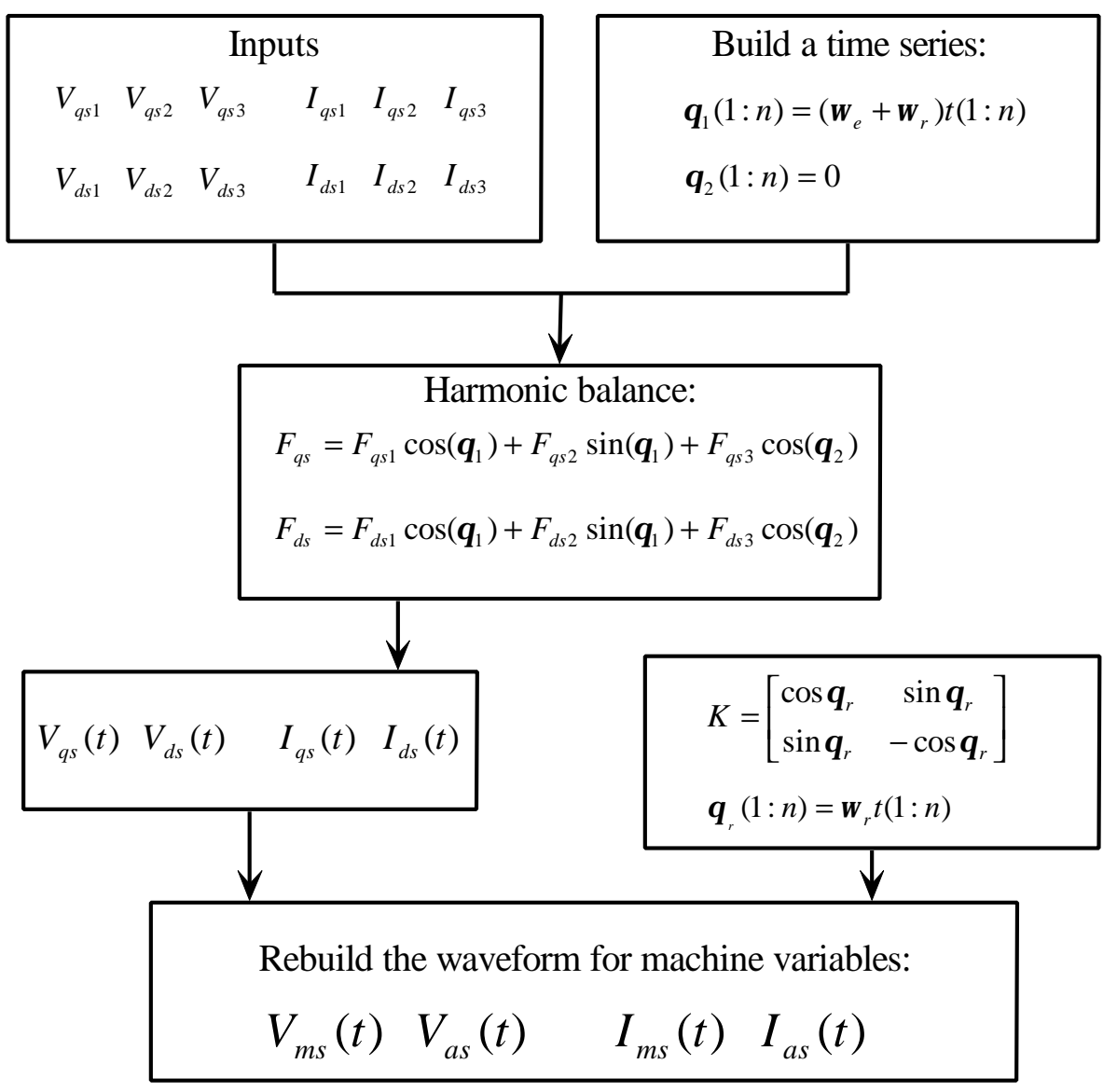

Figure 5-4: Flow chart for waveform reconstruction

With the proposed method, steady state performance will be analyzed. The simulation and experimental results are shown in next chapter. 


\section{CHAPTER}

SIX

\section{Simulation and Experiment Results}

The models provided in previous chapters for both transient and steady state analysis are desirable for a design environment. But only after the simulation and comparing the experiment data with simulation results, the validation of the proposed models can then be proven. This chapter will examine the simulation and present the comparison.

The machine used in this research is a synchronous motor. Its name plate data are in table 6-1.

Table 6-1 Name plate data

\begin{tabular}{|l|l|}
\hline Phase & 2 \\
\hline Rotor poles & 2 \\
\hline frequency & $60 \mathrm{~Hz}$ \\
\hline Stator voltage & $110 \mathrm{v}$ \\
\hline Rotor voltage & $120 \mathrm{v}$ \\
\hline
\end{tabular}

To use this machine as single-phase self-excited reluctance generator, the rotor winding is open circuited. And two stator windings are connected with loads and terminal capacitors.

Figure 6-1 show a picture of the set. 


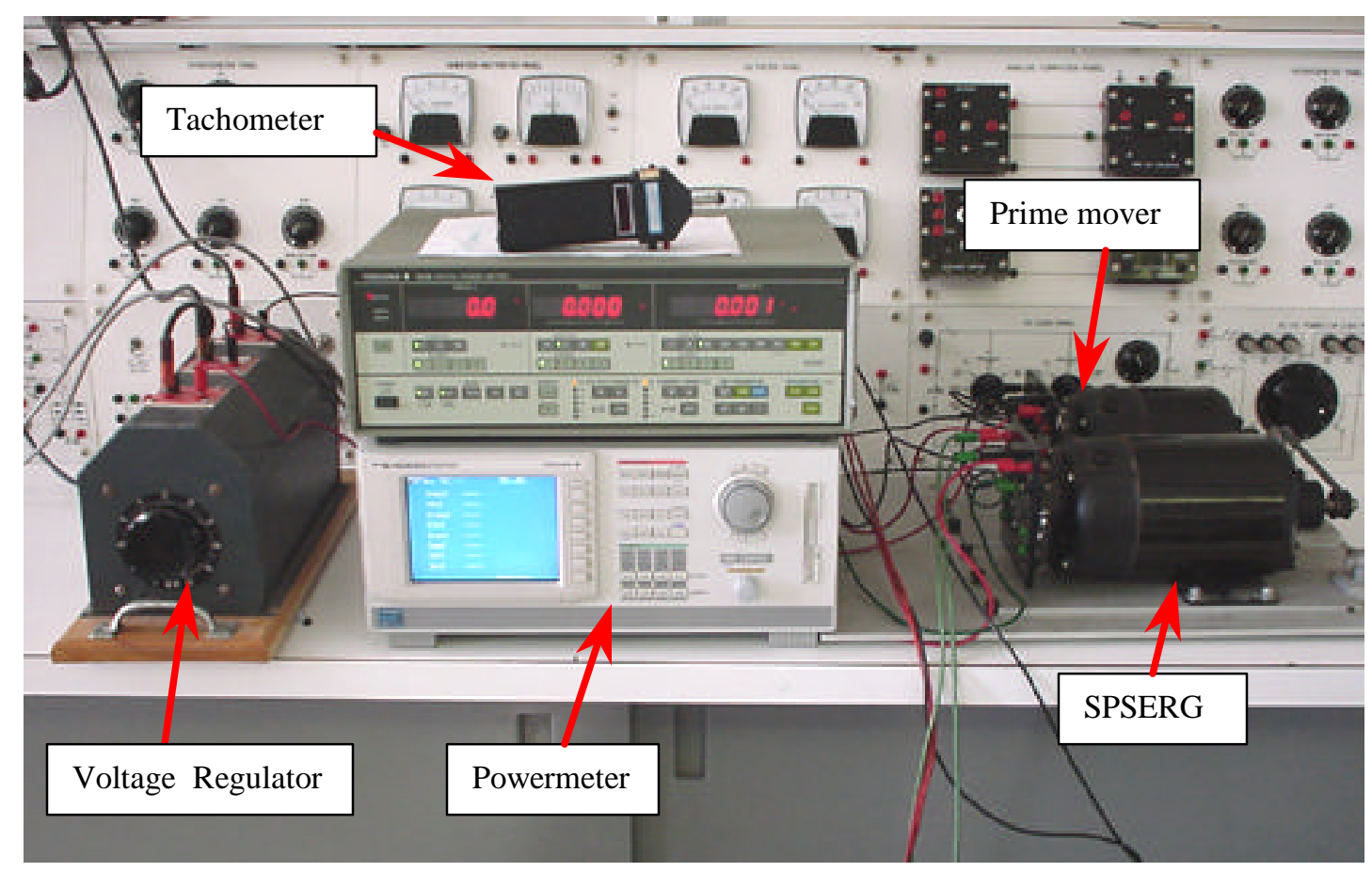

Figure 6-1: Picture of the set

The voltage regulator is employed to adjust the applied voltage to an induction motor which is operated as the prime mover. A tachometer is utilized to measure the rotary speed.

\subsection{Simulation and Experiment for Transient Analysis}

\subsubsection{Simulation Using the Measured Saturation Curves}

The saturation curves for the inductances can be measured by static measurement as shown in Figure 6-2. Basing on this measurement, machine model under natural machine variables can be applied to simulate the transient process. 


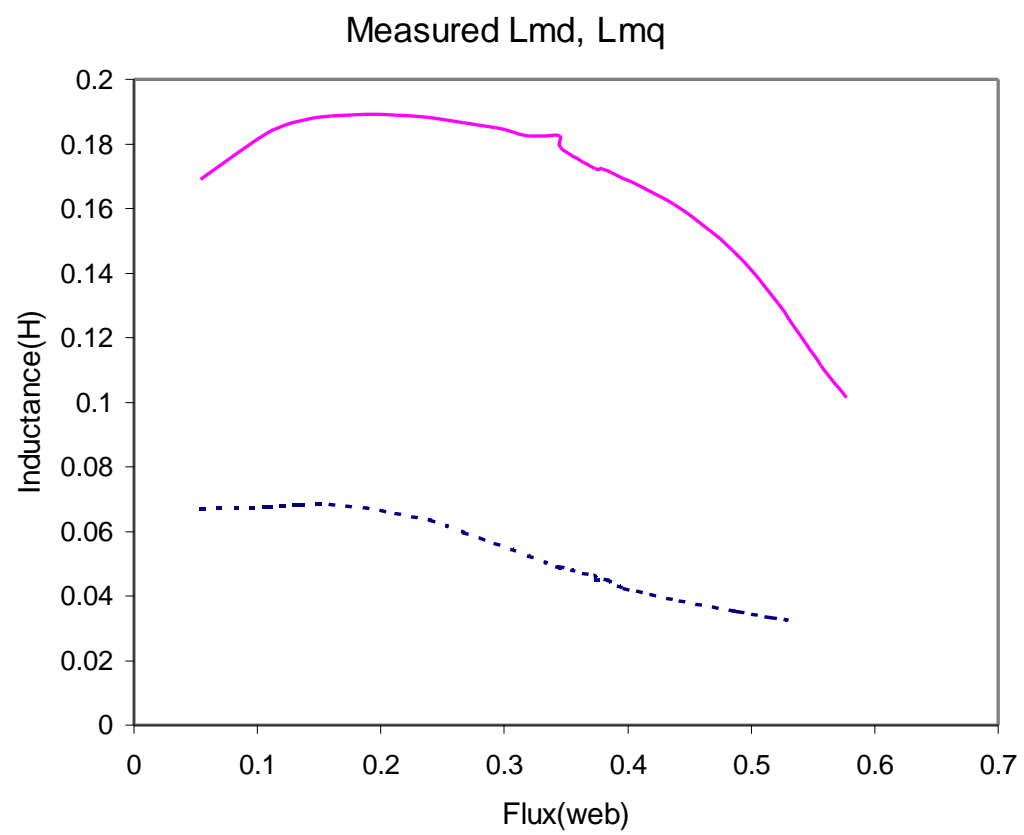

Figure 6-2: Measured inductances Lmd (solid) Lmq (dotted)

Figure 6-3 to 6-7 show a simulation example for SPSERG under pure resistive load. Simulation Condition: $C_{m}=127 \mu f, C_{m}=267 \mu f, R_{L}=100 \Omega, \omega_{r}=188.5 \mathrm{rad} / \mathrm{s}$

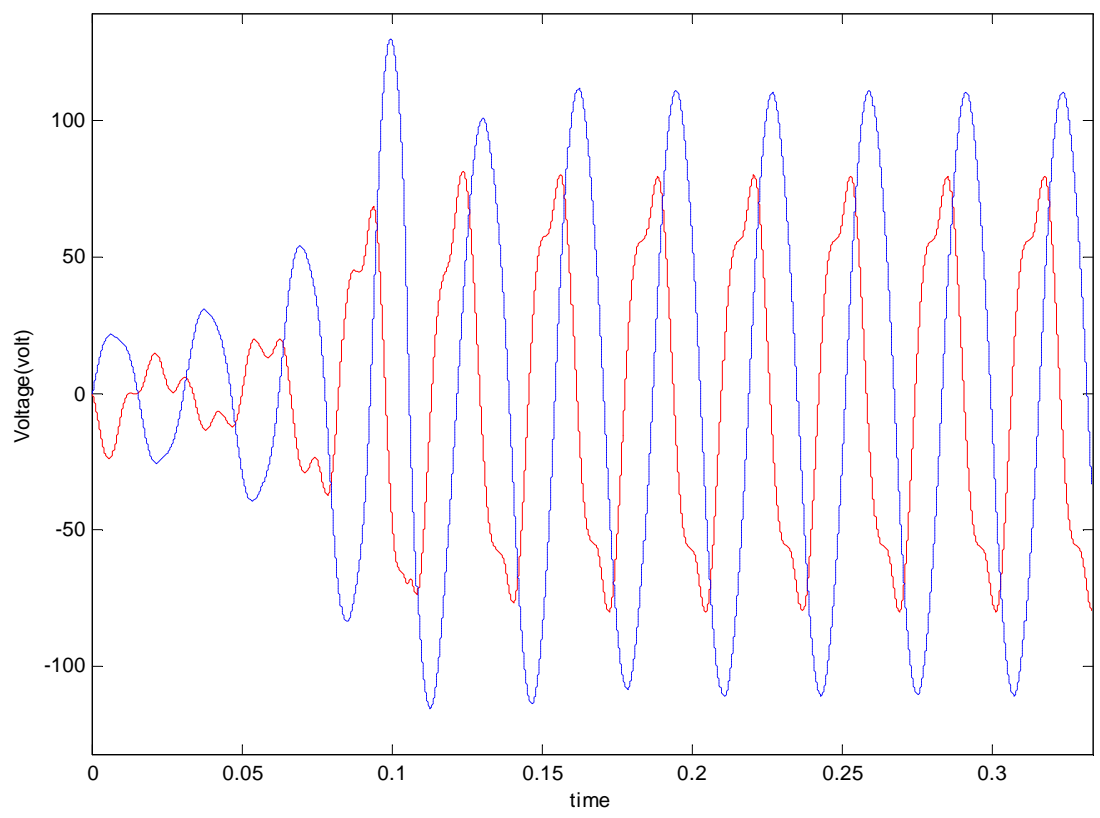

Figure 6-3: Output voltages $V_{\text {aux }}$ (dotted), $V_{\text {main }}$ (solid) 


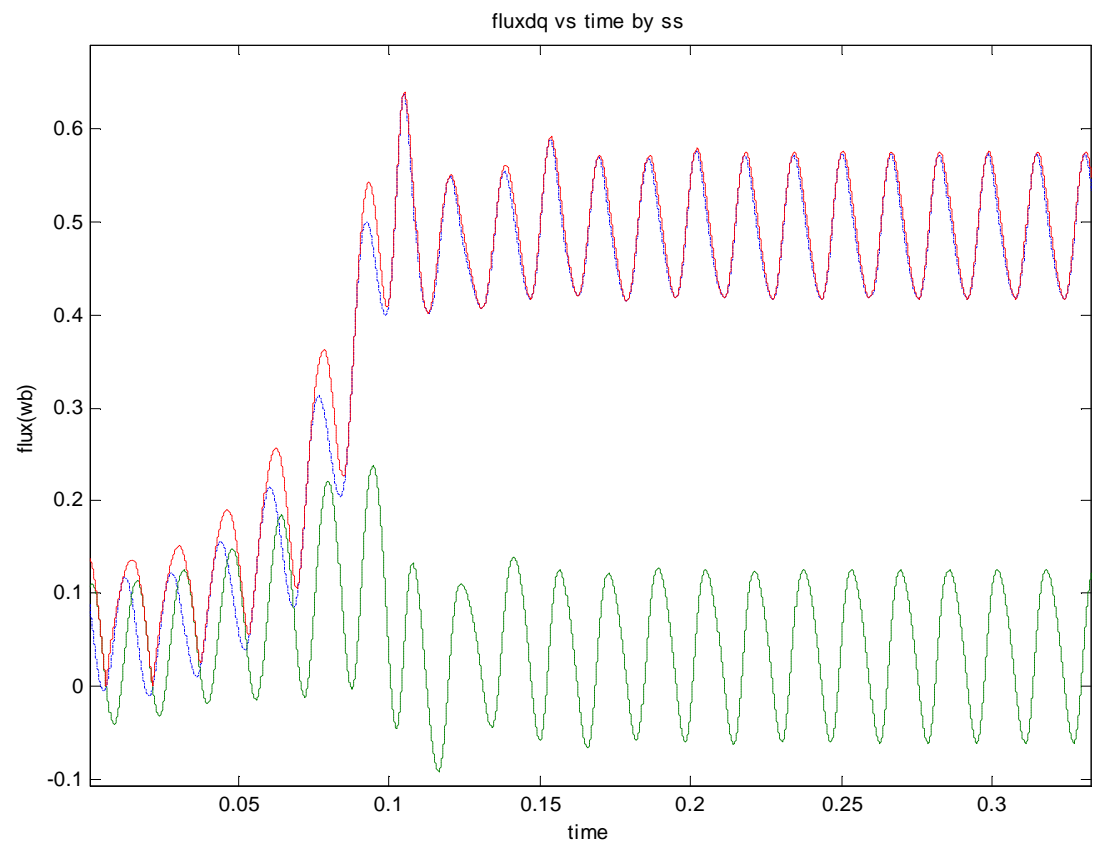

Figure 6-4: Fluxes vs time in qd frame (Main flux — solid, q flux-dotted, d flux—dashed)

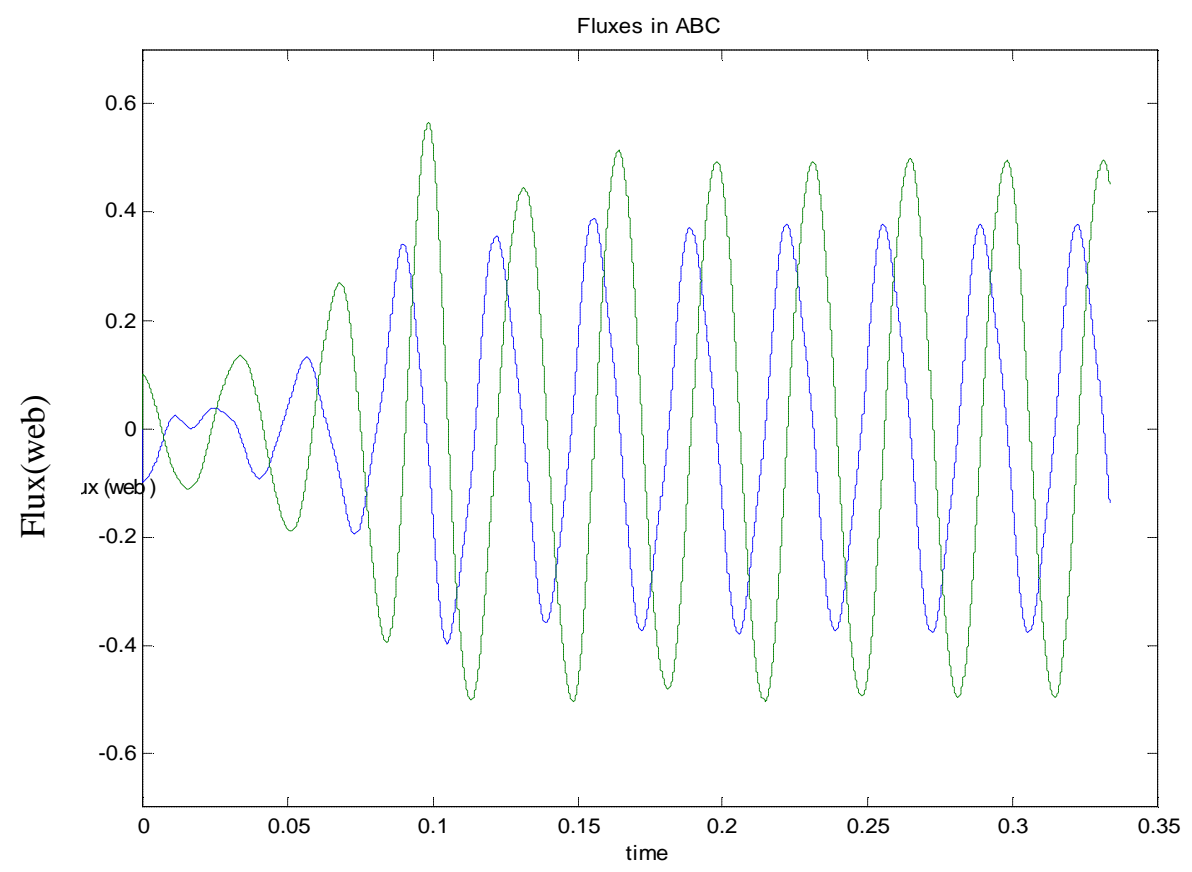

Figure 6-5: Fluxes vs time in abc frame

(flux coupled with main winding—solid, flux coupled with auxiliary winding—dotted) 


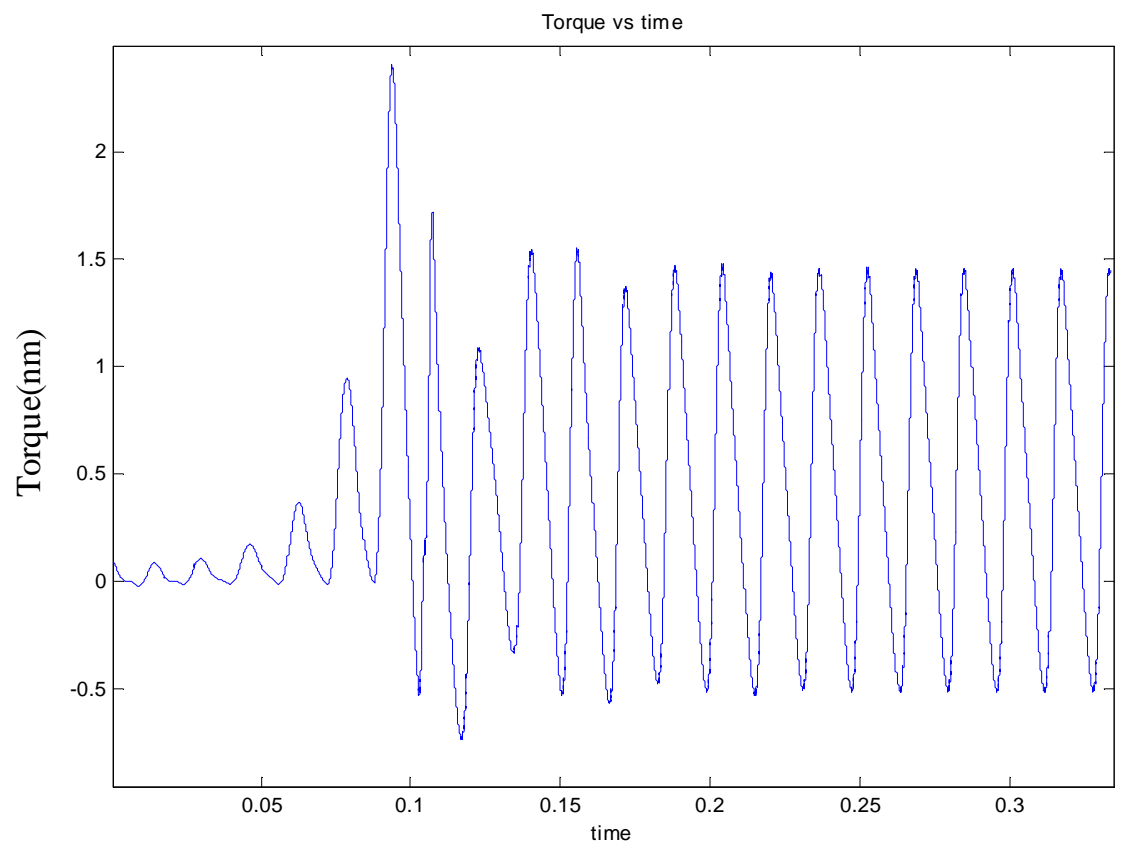

Figure 6-6: Electromagnetic torque vs time

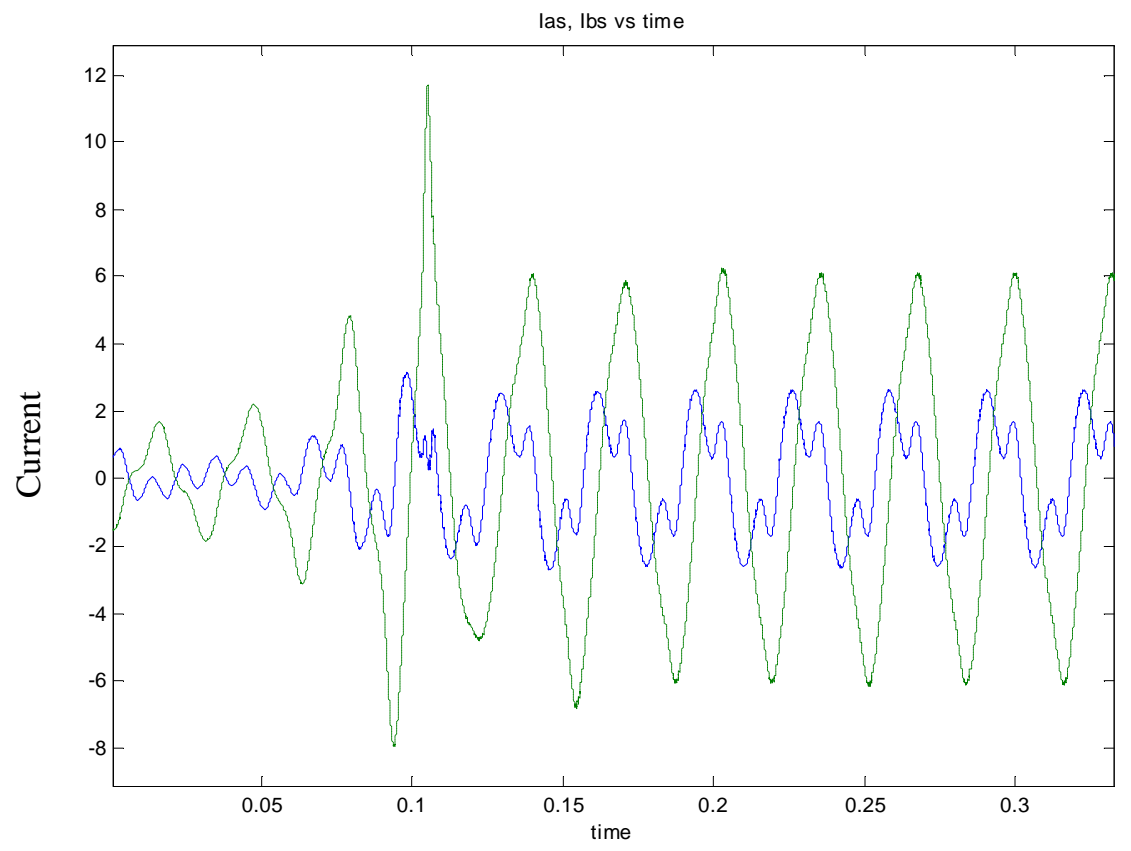

Figure 6-7: Output currents $I_{\text {aux }}$ (dotted), $I_{\text {main }}$ (solid) 


\subsubsection{Inductance profile analysis}

Before any analysis is given, understanding of the inductance saturation is essential, for it directly reflects the nonlinear nature of the system. From the basic definition, the inductance matrix can be expressed as:

$$
L=\left[\begin{array}{cc}
L_{l}+L_{a}^{\prime}+L_{a}^{\prime \prime} \cos \left(2 \theta_{r}\right) & L_{a b} \sin \left(2 \theta_{r}\right) \\
L_{a b} \sin \left(2 \theta_{r}\right) & L_{l}+L_{b}^{\prime}-L_{b}^{\prime \prime} \cos \left(2 \theta_{r}\right)
\end{array}\right]
$$

In steady state analysis, this expression can be simplified as a balance case by assuming:

$$
L_{a}^{\prime}=L_{b}^{\prime}=L^{\prime} \quad L_{a}^{\prime \prime}=L_{b}^{\prime \prime}=L_{a b}=L^{\prime \prime}
$$

Therefore, the new inductance is:

$$
L=\left[\begin{array}{cc}
L_{l}+L^{\prime}+L^{\prime \prime} \cos \left(2 \theta_{r}\right) & L^{\prime \prime} \sin \left(2 \theta_{r}\right) \\
L^{\prime \prime} \sin \left(2 \theta_{r}\right) & L_{l}+L^{\prime}-L^{\prime \prime} \cos \left(2 \theta_{r}\right)
\end{array}\right]
$$

Using the reference frame theory, the inductance under rotor reference frame is:

$$
L=\left[\begin{array}{cc}
L_{l}+L^{\prime}+L^{\prime \prime} & 0 \\
0 & L_{l}+L^{\prime}-L^{\prime \prime}
\end{array}\right]=\left[\begin{array}{cc}
L_{l}+L_{m d} & 0 \\
0 & L_{l}+L_{m q}
\end{array}\right]
$$

This new inductance matrix is only determined by the machine structure and the simplification assumption, but independent of the terminal connections and loads. The saturation can directly be reflected in $L_{m d}, L_{m q}$. This is critical for the qd frame analysis, because it guarantees the rotor reference theory can be valid no matter what other considerations such as saturation, unbalance load are integrated.

Then the unbalance load can be embedded in $L_{m d}$ and $L_{m q}$ as a saturation effect.

An interesting phenomenon is observed here. Because of the saturation, the inductance matrix is not balance, even though the reference frame theory is still valid. 


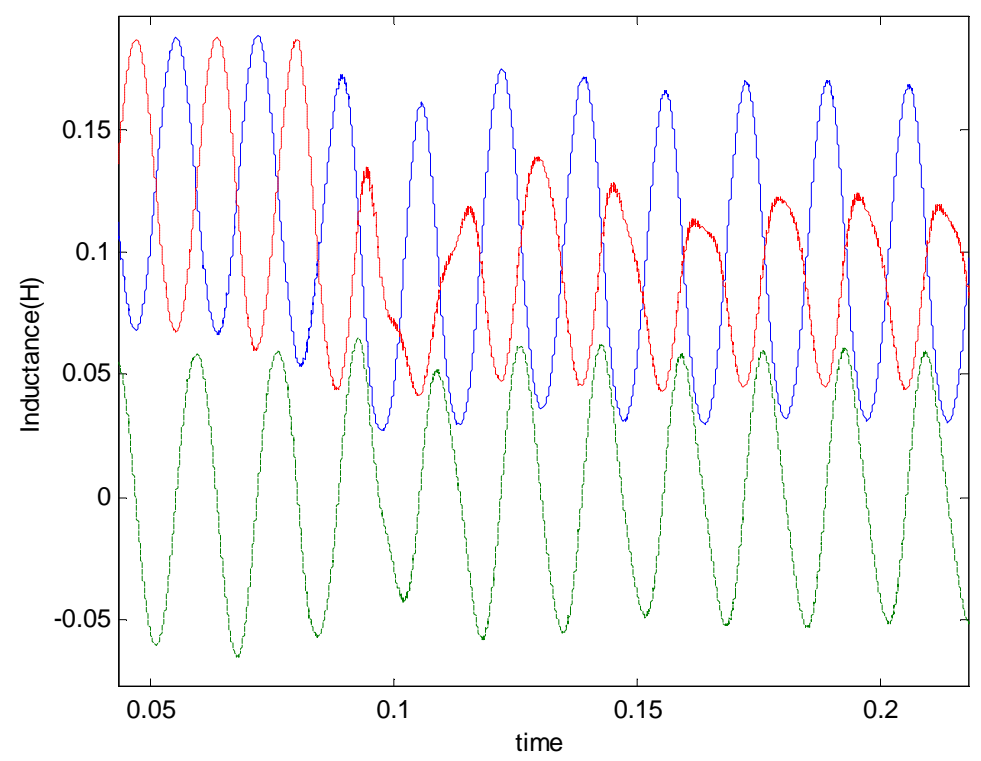

Figure 6-8: Inductance profile during the whole self excitation process

( Solid-self inductance of main winding, Dotted-- self inductance of auxiliary winding,

$$
\text { Dash-mutual inductance) }
$$

It is interesting to see from Figure 6-8, which describes the inductance profiles in the transient response. At the starting period, the load and auxiliary winding currents are small, which can be treated as a linear condition. During this period, the self-inductances for the main and auxiliary windings have the same DC and AC amplitudes and $180^{\circ}$ phase shift, which are two balance profiles. But under steady state condition, because of the nonlinear nature of this system, saturation must be present. Consequently, the inductance profiles for the main winding and auxiliary winding are unbalance, which means that the saturation effect on the two axes is not symmetric. In the d-axis, because of the small air gap, the saturation is far more serious than that in q-axis, whose inductance is almost a constant value. Consequently, combined with the time variant load, the inductance in d-axis is shown in Figure 6-9 as $L_{m d}$. 
Because the oscillation of the saturated $L_{m d}$ is in the same phase with the main winding self inductance and $90^{\circ}$ delay to the mutual inductance, their amplitudes are amplified, while the self inductance for the auxiliary winding has $180^{\circ}$ phase difference from $L_{m d}$, which chops its peak and results in an unbalance smaller self inductance.

This phenomenon indicates that even tough the two stator windings are balance distributed, because of the unbalance load and the relevant saturation effect, they can still display unbalance inductance distribution. But it should be noted that at every point, equation (6-2) is still valid, and so as equation (6-3).

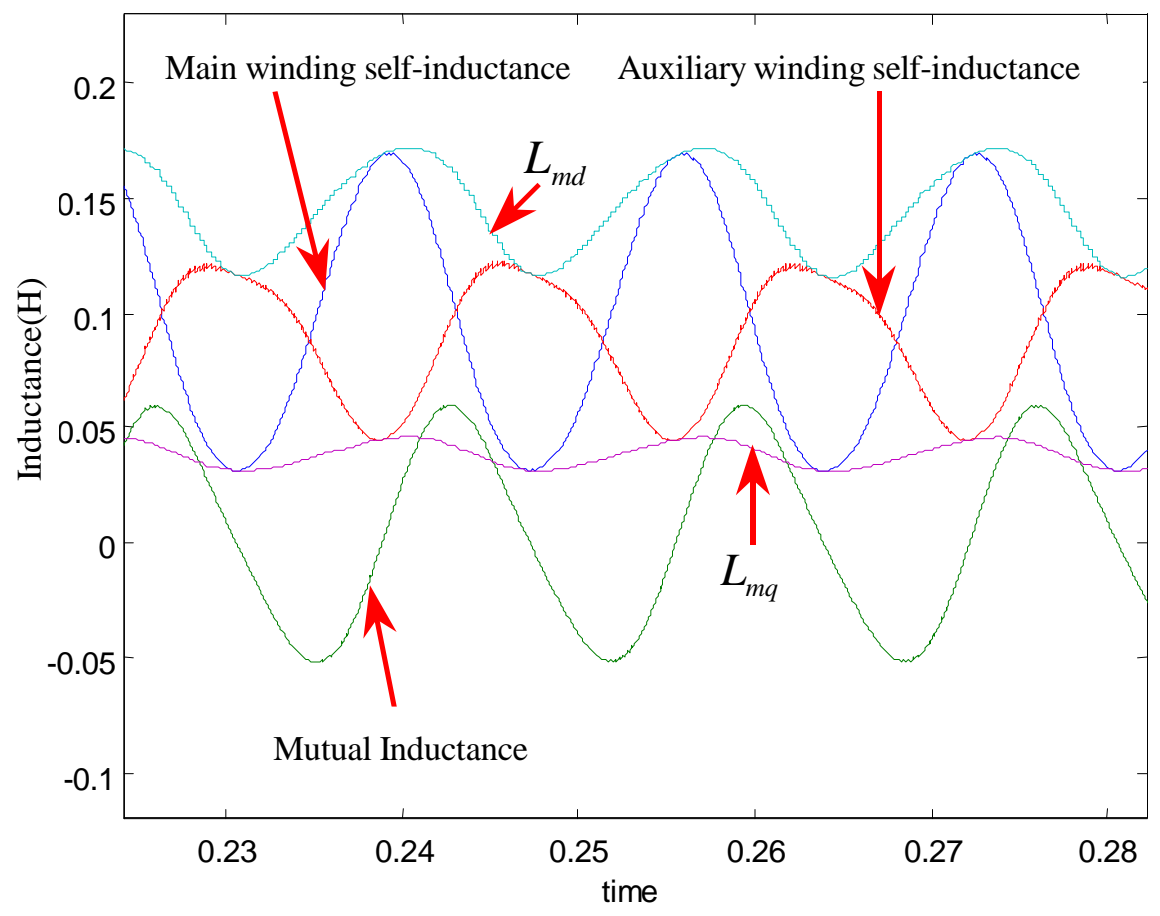

Figure 6-9: Inductance profile

In analysis under QD reference frame, it is a common adopted method for nonlinear analysis to lump the saturation effect to d axis. But the impact to the real machine inductances has not been stated. In this case, because of the unbalance load 
condition, $\mathrm{ABC}$ model is more suitable to be taken to simulate the dynamics, which reveals the saturation effect to the machine inductance variables.

\subsubsection{Transient Simulation Using Computed Saturation Curves}

As explained in Chapter 4, the measured saturation curves are averages of inductance vs flux. Even though they only reflect the saturation in rough accuracy, the relationship between flux and inductances are monotonous, and can be easily integrated into the state space simulation. However, for the instantaneous inductances obtained from FEM, although they are more accurate, there is no way to represent the relationship between flux and inductance in distinct expressions. That means that especially at high

flux region, one flux corresponds to two inductances and no criteria can help selecting one of them as the physical inductance.

For design environment, FEM is still the best way to obtain the inductances. Therefore, as proposed in Chapter 4, computed saturation curves are established to replace the measured saturation curves.

Comparison between computed saturation curve and measured curves are shown in Figure 6-10 and 6-11. 


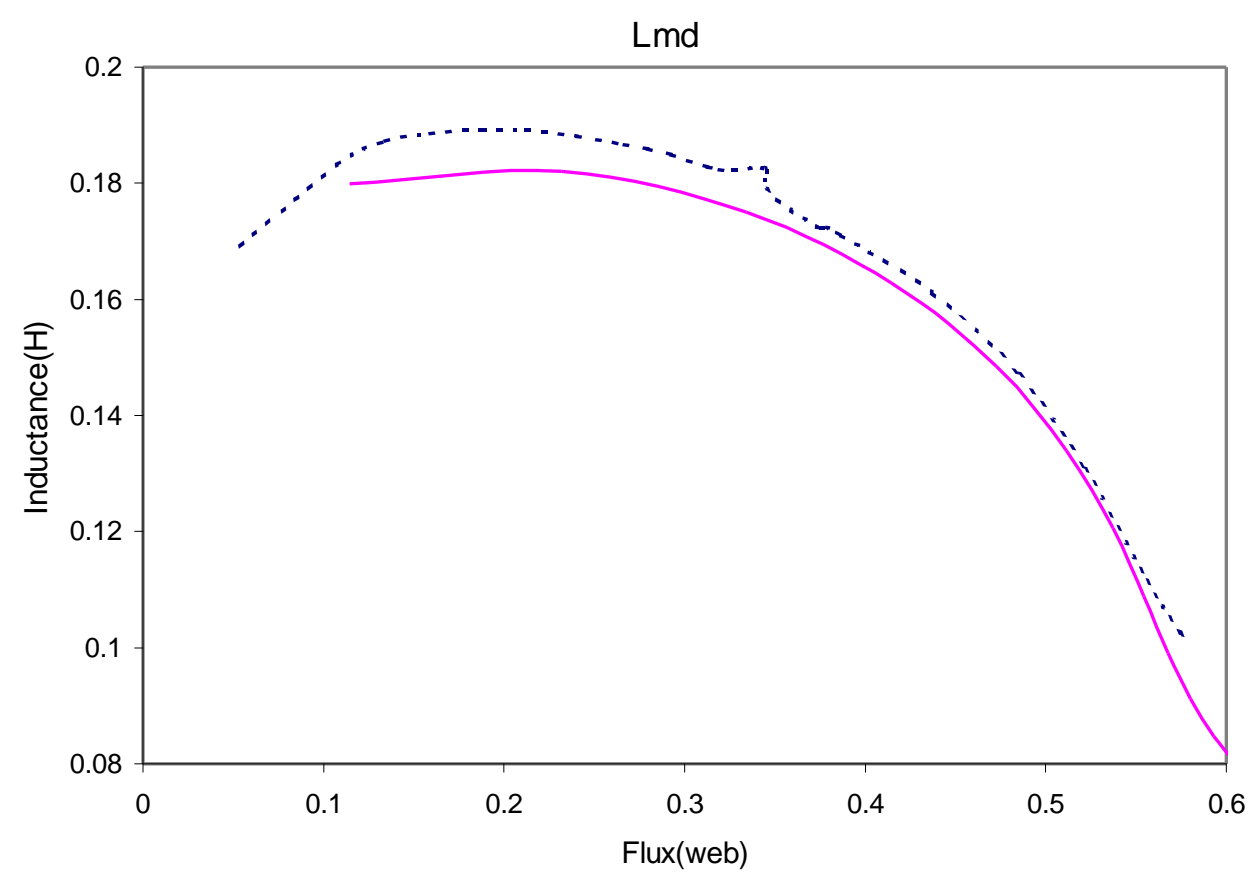

Figure 6-10: Comparison of the Lmd by experiment and FEM

(dotted—experimental result, solid—computed curve)

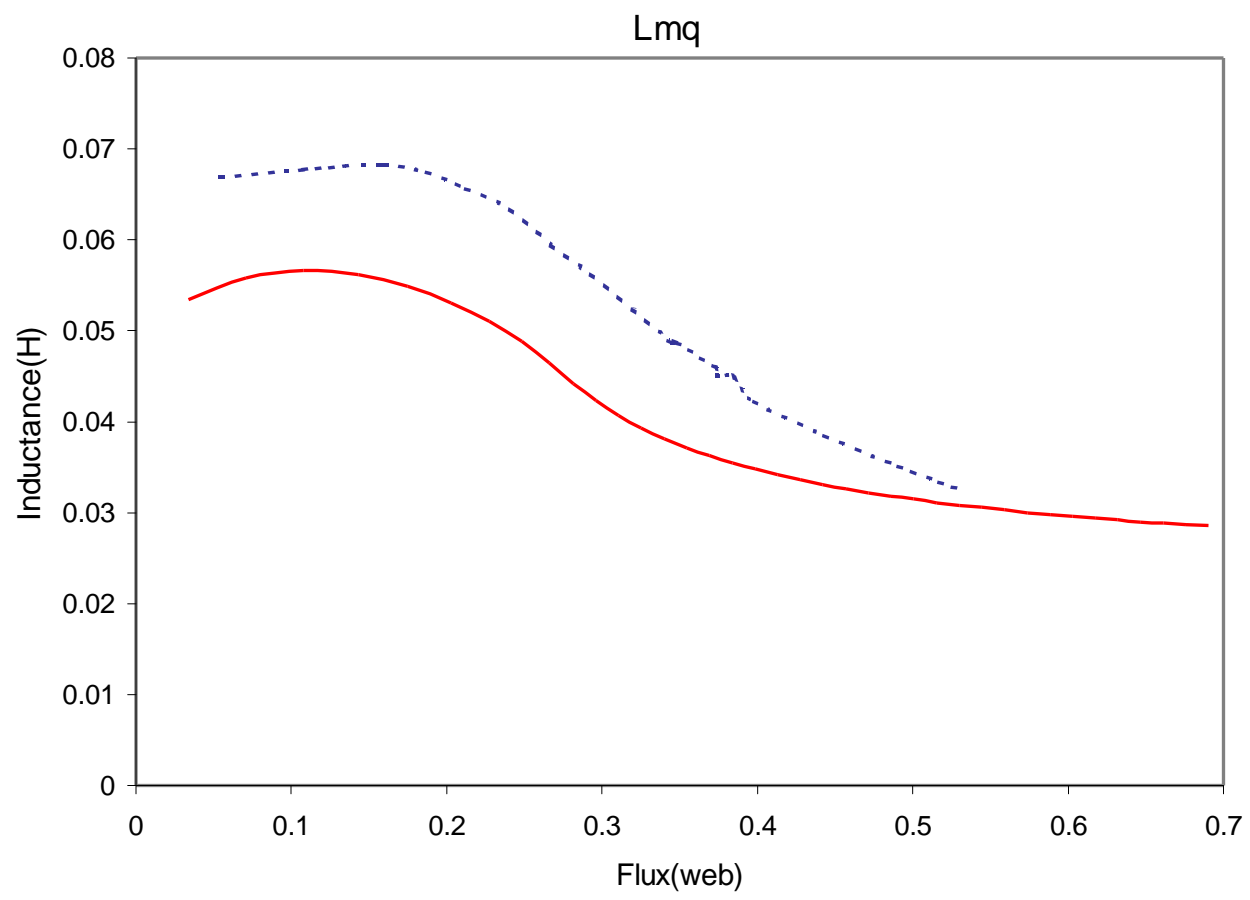

Figure 6-11: Comparison of the Lmq (Dotted—experimental result, solidcomputed curve) 
The 2-D FEM cannot solve the terminal leakage inductance, which is in nature a 3-D configuration. Therefore, the difference between the inductance obtained by FEM and the measured inductance indicates the leakage inductance.

Basing on the computed saturation curves, the simulation results are compared with the experiment results. The comparison of the state variables is shown in Figures 612 and 6-13, where $C_{m}=127 \mu f, C_{m}=267 \mu f$, pure resistive load of $R_{L}=100 \Omega$.

From the comparisons, the proposed transferred inductance approach for transient response can represent the system characteristics in good accuracy.

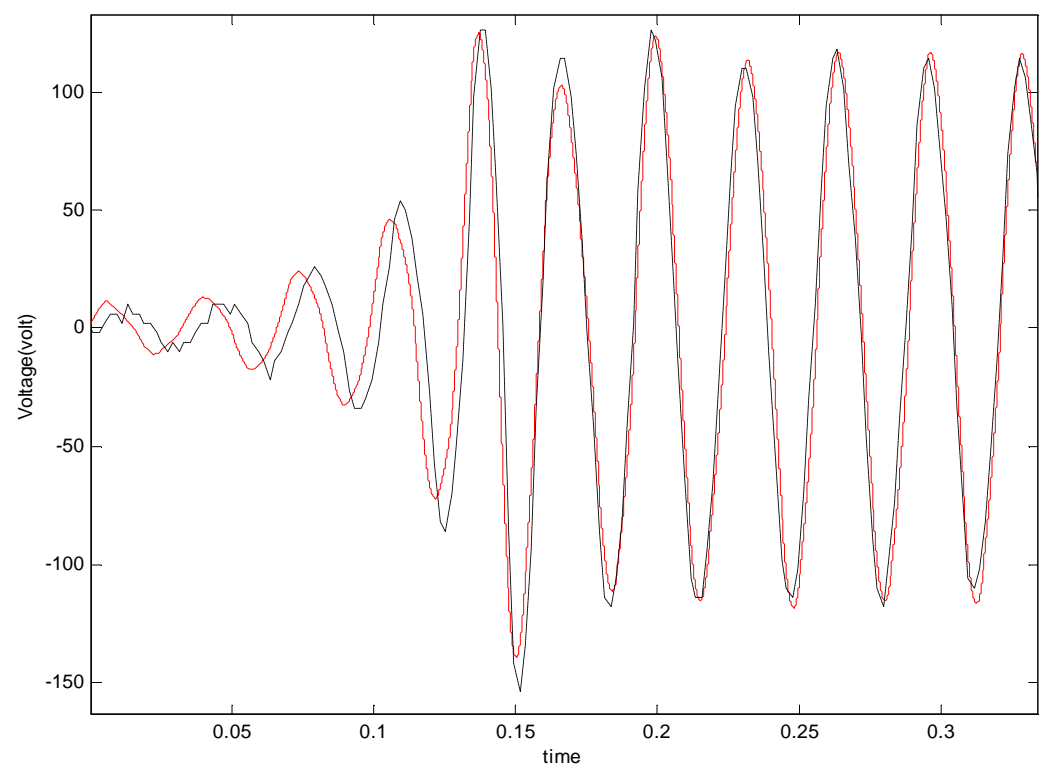

Figure 6-12: Comparison of the auxiliary winding terminal voltage (Dotted—experiment result, solid—simulation result) 


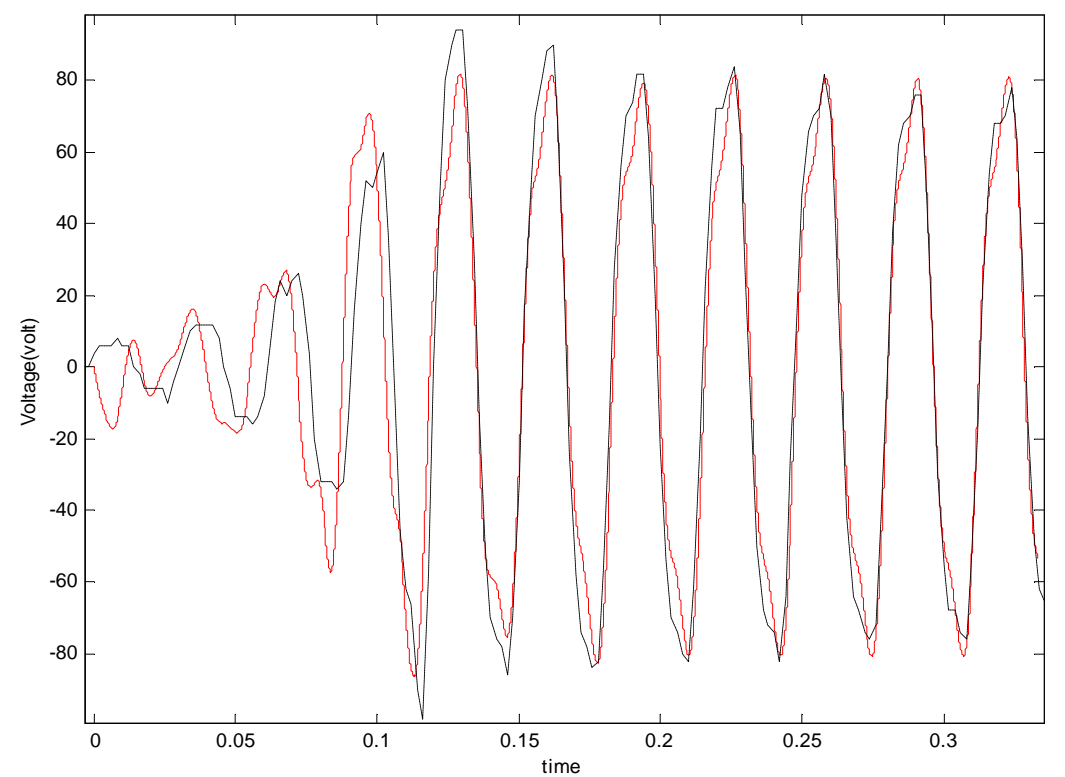

Figure 6-13: Comparison of the main winding terminal voltage

(Dotted—experiment result, solid—simulation result)

The discrepancy in the transient response is from two sources. One is the prime mover modeling. Because in this case, the prime mover is a small $1 / 2 \mathrm{hp}$ induction motor, who's dynamic should be coupled with the SPSERG. But because of the inadequate information of the motor inertia, the dynamic of the induction motor is neglected, which, as a result, will generate error. The other reason is the initial condition. The initial condition, mainly the residual flux, for the simulation cannot be the same with the actual unknown residual flux. Therefore, the starting period of the transient response cannot be identical with the experimental results.

To examine the different responses between the measured and computed saturation curves, the comparison of the simulation results by these two approaches are shown in Figure 6-14 and Figure 6-15, where $C_{m}=127 \mu f, C_{m}=267 \mu f$, pure resistive load $R_{L}=100 \Omega$. 


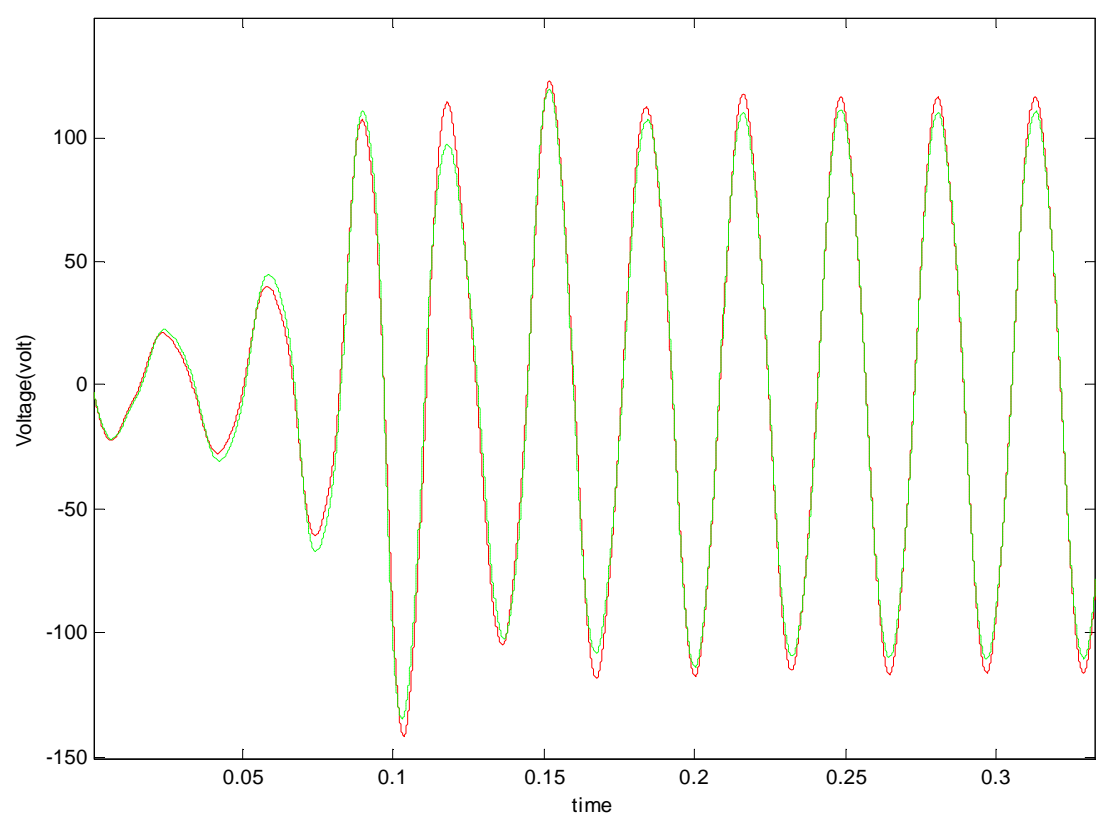

Figure 6-14: Comparison of the auxiliary winding terminal voltage

(Dotted — with measured saturation curves, solid—computed saturation curves)

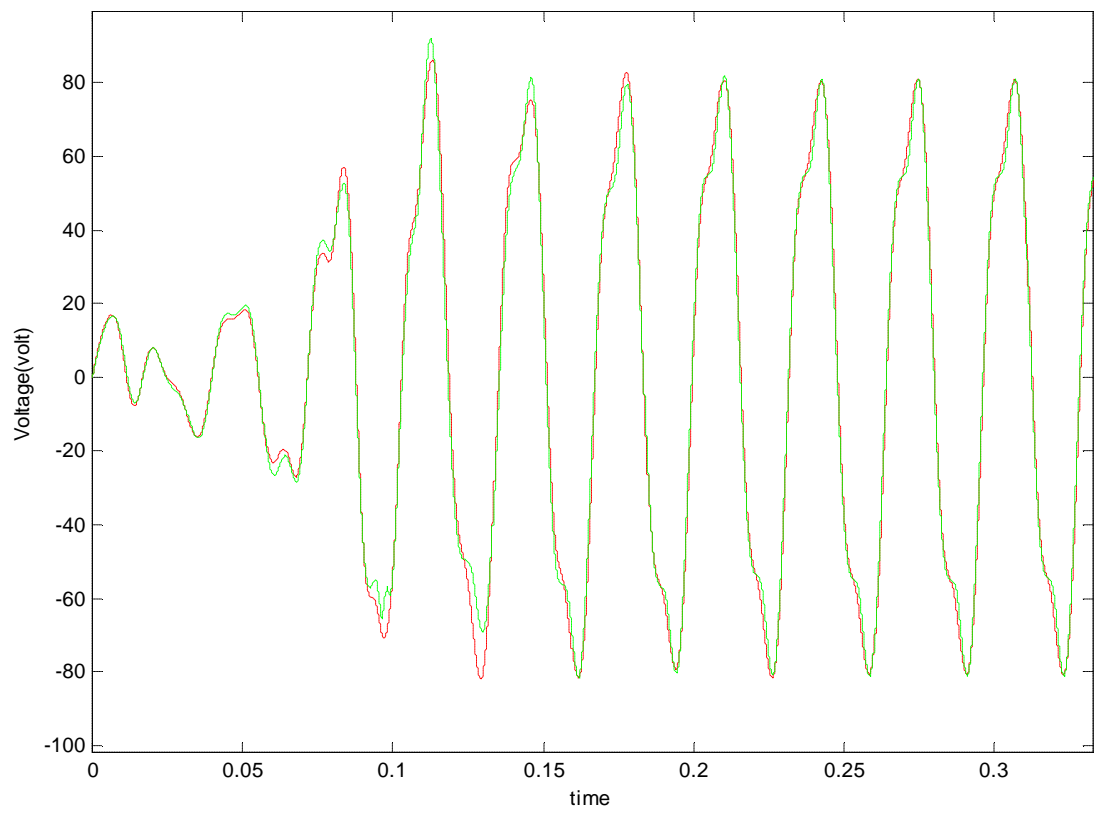

Figure 6-15: Comparison of the main winding terminal voltage

(Dotted—with measured saturation curves, solid—computed saturation curves) 
It shows that from the same initial condition, the terminal voltages have very close profiles.

Furthermore, a more general RL load is simulated. And the comparison of the state variables between experiment and simulated results is shown in Figure 6-16 and 6-17, where $C_{m}=127 \mu f, C_{m}=267 \mu f, L_{m}=133 m H, R_{L}=179 \Omega$. It is observed that the discrepancy is higher than pure resistive load. This may come from the parasitic capacitance or the motor mechanical dynamic, which is not fully considered.

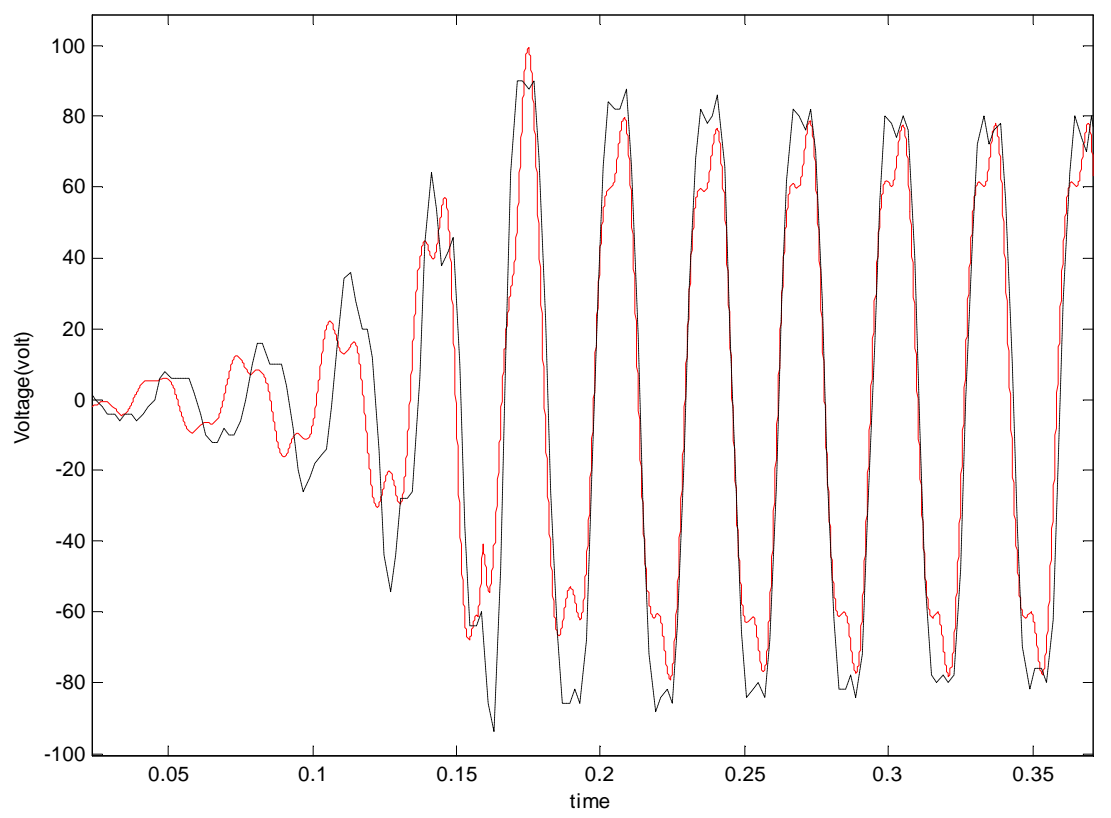

Figure 6-16: Comparison of the main winding terminal voltage (Dotted—experiment result, solid—simulation result) 


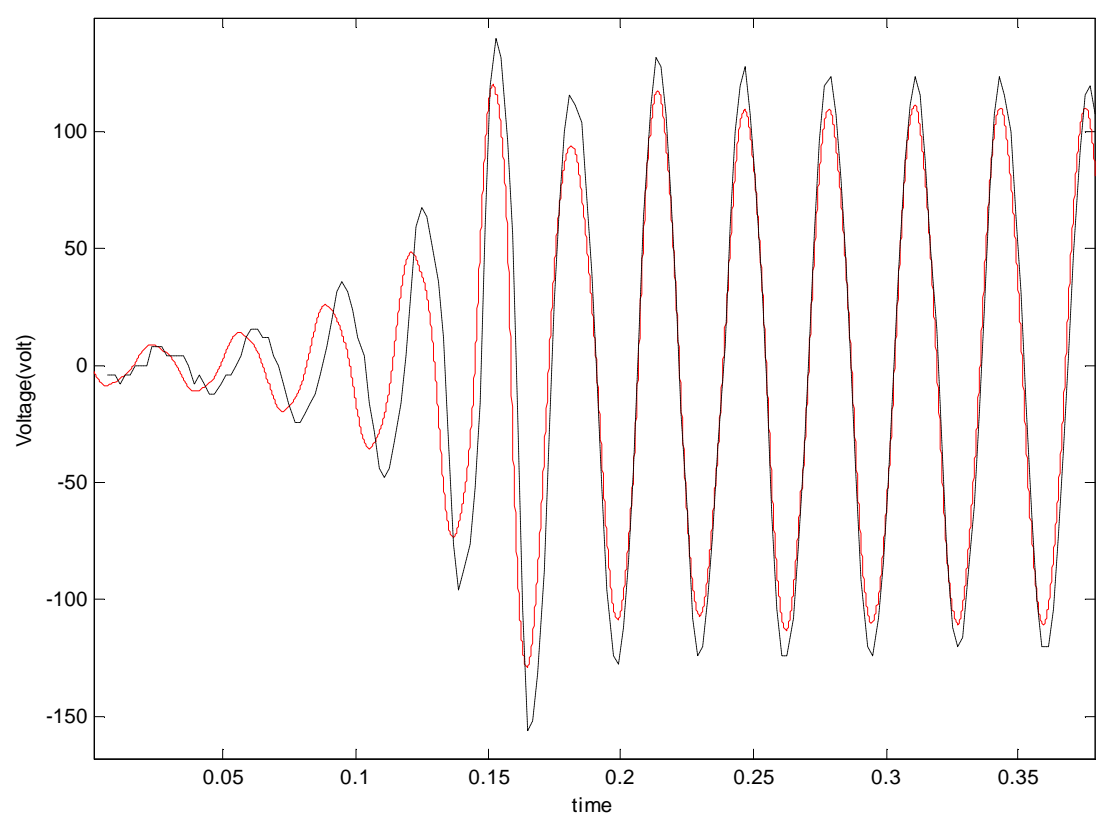

Figure 6-17: Comparison of the auxiliary winding terminal voltage

(Dotted-experiment result, solid—simulation result)

\subsection{Simulation and Experiment for Steady State Analysis}

If the steady state model is correct, it can be used to reveal the relationship between variables. Moreover, it can also be used to reconstruct the variable waveform in time domain. So in this section, not only the steady state performances are investigated, but the state variables are also reestablished to validate the approach.

\subsubsection{Waveform Analysis}

In the simulation, the steady state method proposed in Chapter 5 is used.

Using the proposed method for steady state analysis, simulation results are given in the following according to different cases. Compared to the experimental results, the 
proposed method displays identical waveform for the main and auxiliary winding output voltages.

\section{Case 1:}

The parameters are pure resistive lad $R_{L}=179 \Omega, C_{m}=99 \mu F, C_{b}=236 \mu F$. In the Figures 6-18 and 6-19, dotted line represents experimental result, and solid line describes the simulation result obtained from the proposed steady state model. It is apparent that the simulated main winding voltage is higher than the experimental result and the auxiliary winding voltage is lower than the experimental result, which are identical with the performance analysis discussed in the next section.

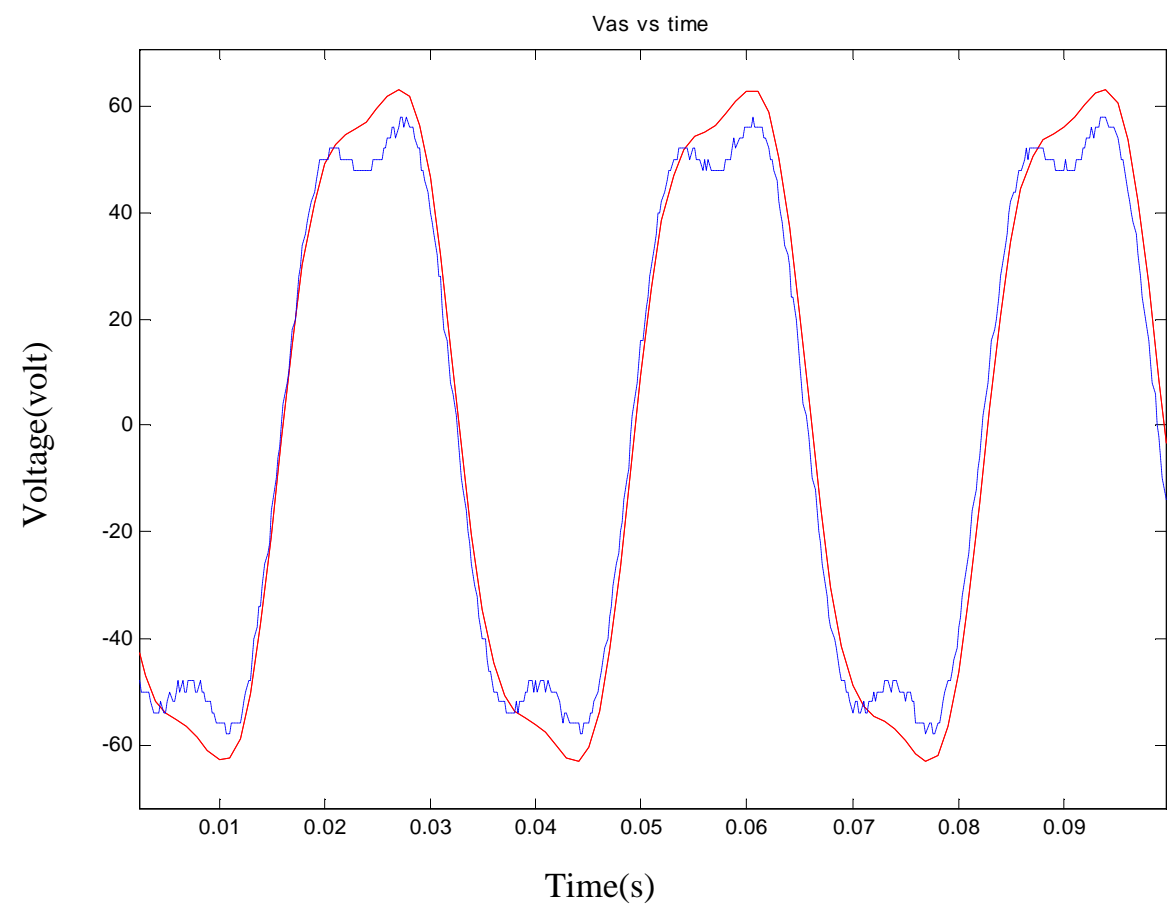

Figure 6-18: Main winding terminal voltages

(Dotted—experiment result, solid—simulation result) 


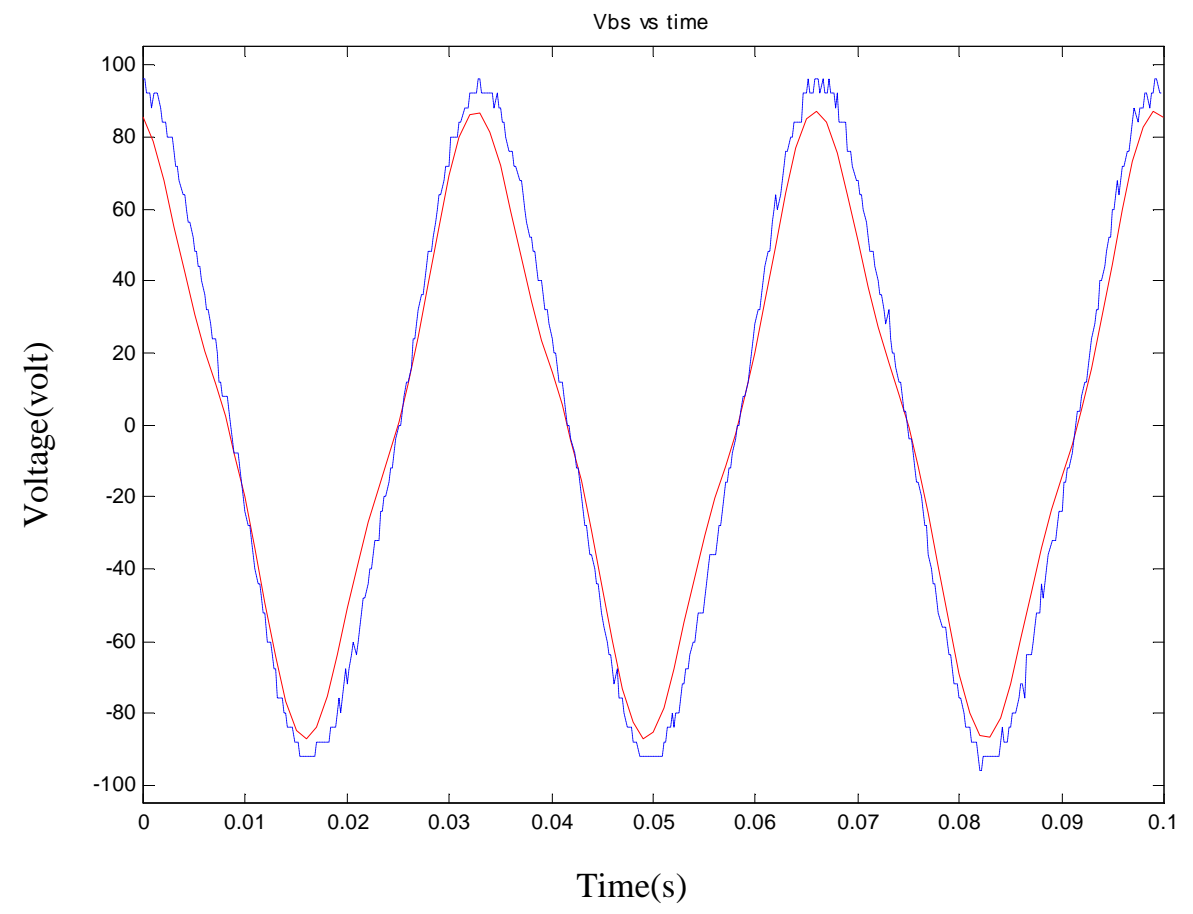

Figure 6-19: Auxiliary winding terminal voltages

(Dotted—experiment result, solid—simulation result)

\section{Case 2:}

The following two Figures 6-20 and 6-21 show the comparison of the simulation results between steady state model and state space model. The parameters for pure resistive load are $R_{L}=110 \Omega, C_{m}=125 \mu F, C_{b}=250 \mu F$. In this case, dotted line represents simulation result obtained from state space model, while solid line describes the simulation result attained from the steady state model.

\section{Case 3:}

To validate the steady state model for RL load, the same approach is also applied. In Figure 6-22 and 6-23, simulation results are compared with the experimental results. The parameters are $C_{m}=127 \mu f, C_{m}=267 \mu f, L_{m}=133 m H, R_{L}=179 \Omega$. 


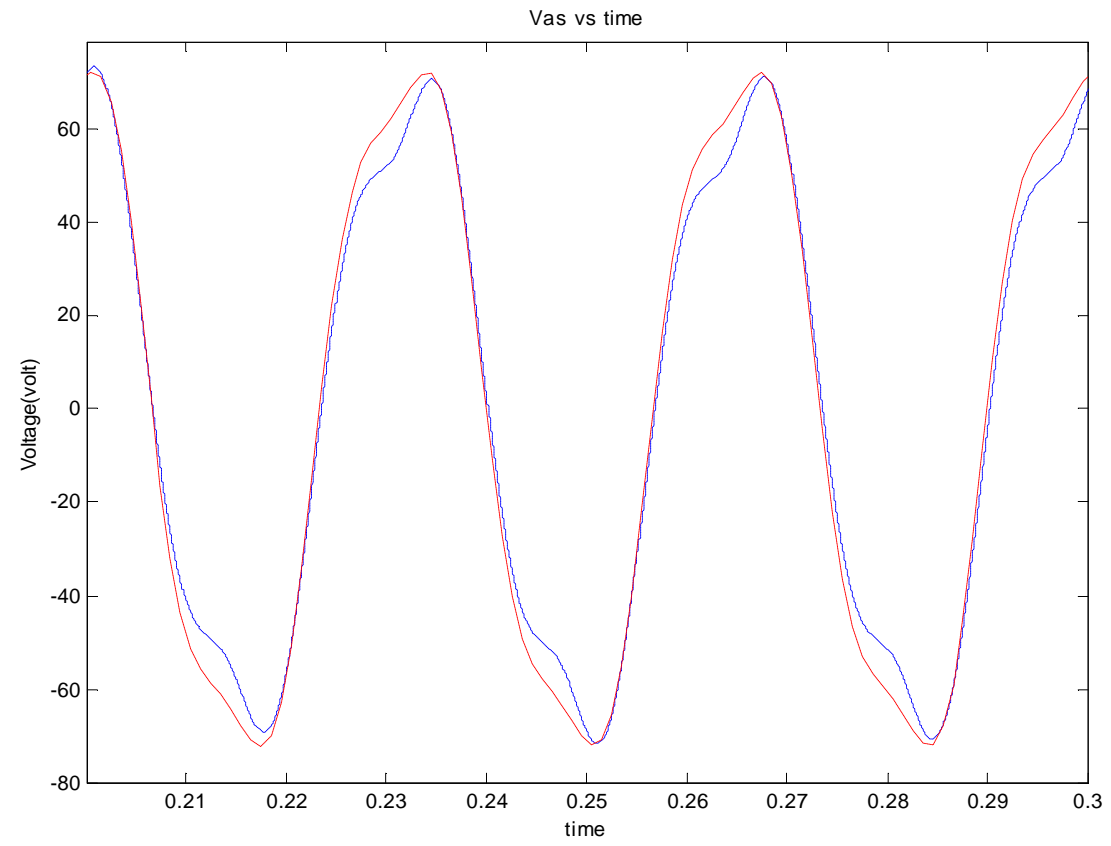

Figure 6-20: Main winding terminal voltages

(Dotted—experiment result, solid—simulation result)

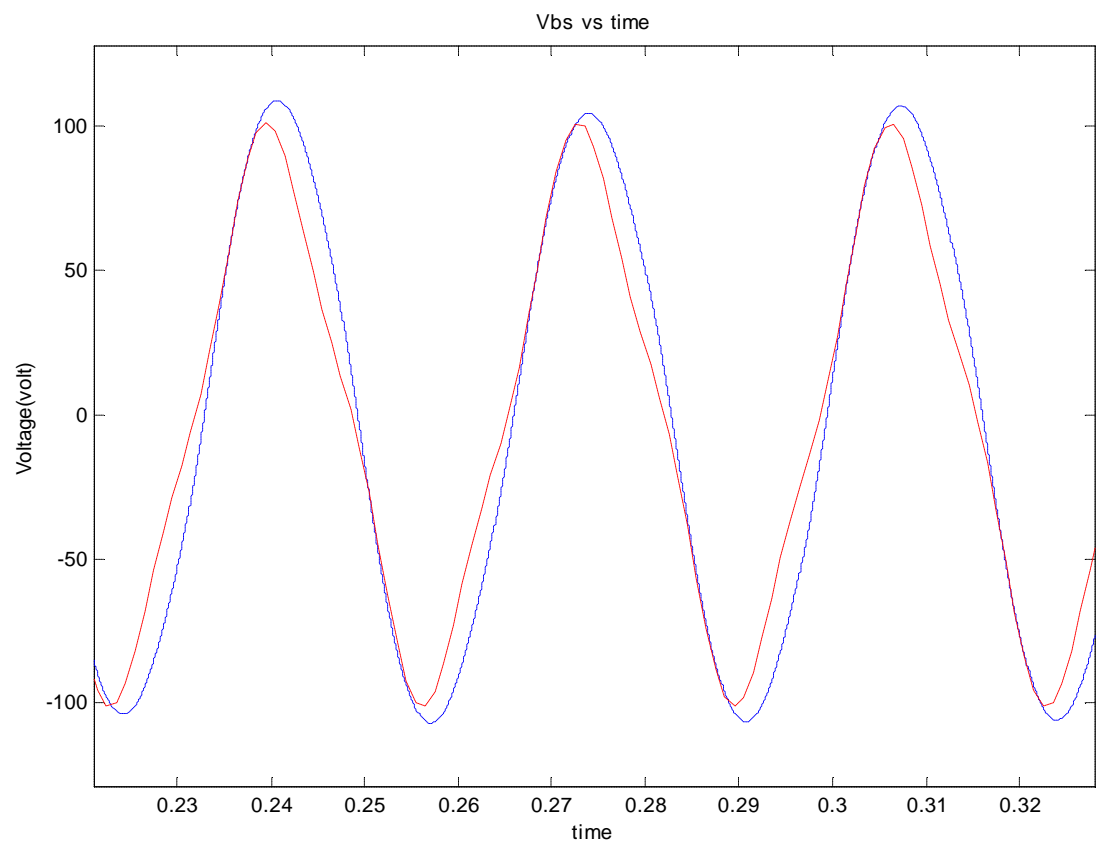

Figure 6-21: Auxiliary winding terminal voltages

(Dotted—experiment result, solid—simulation result) 


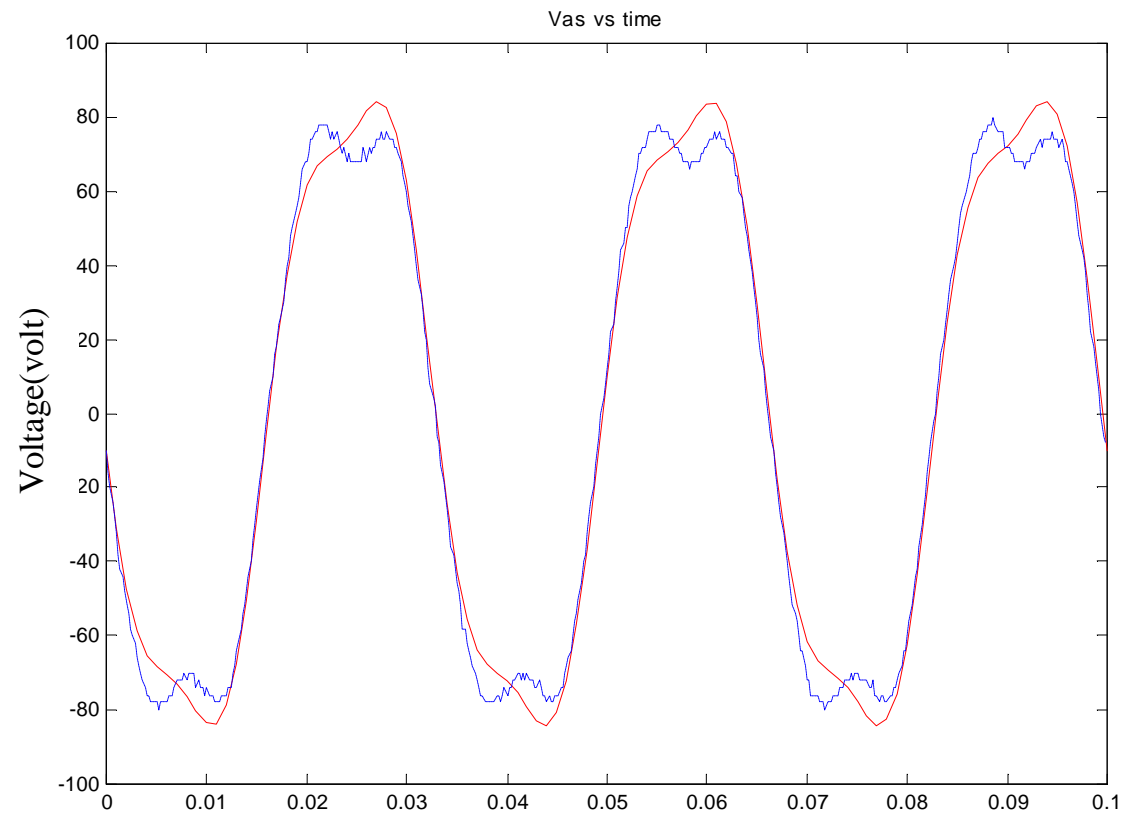

Time(s)

Figure 6-22: Main winding terminal voltages

(Dotted—experiment result, solid—simulation result)

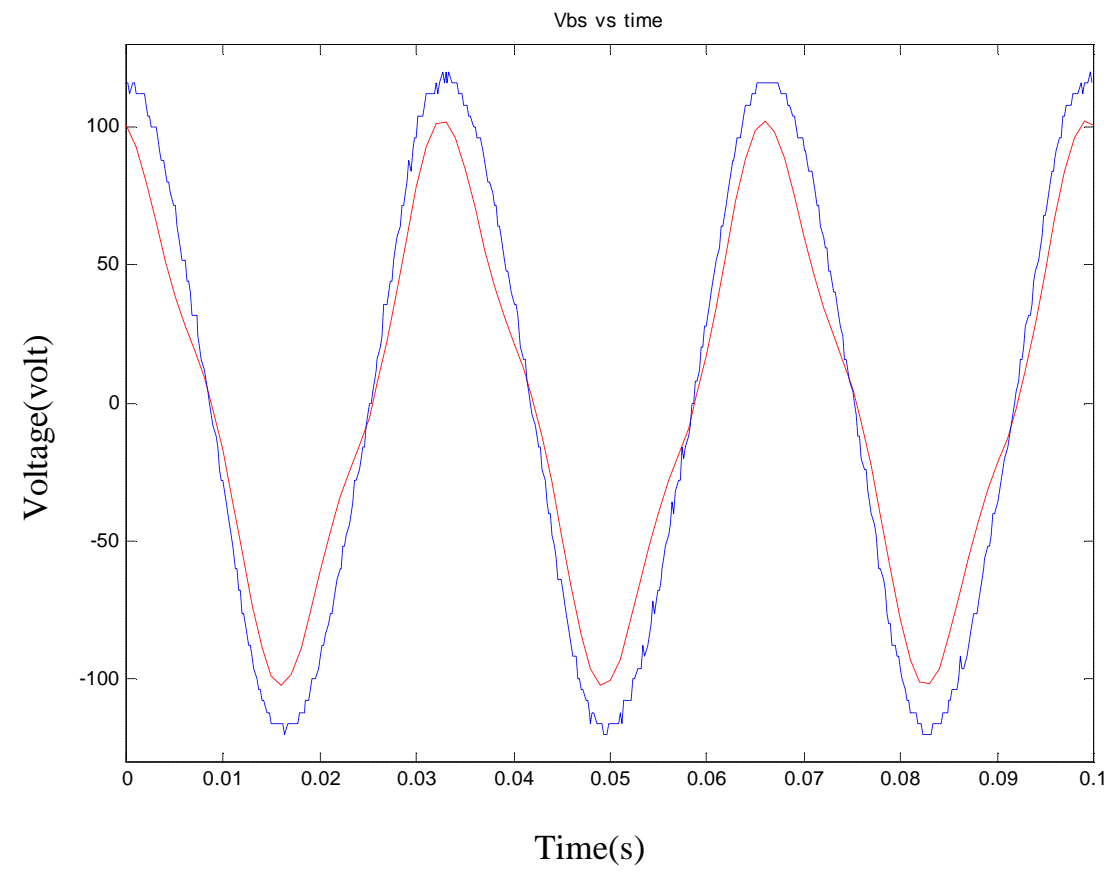

Figure 6-23: Auxiliary winding terminal voltages

(Dotted—experiment result, solid—simulation result) 
From the above comparison under different cases, it is apparent that the reconstruction of the waveform can help visualizing the time domain information, which can in turn provide a better understanding of the detailed trajectory of the system variables.

\subsubsection{Performance Analysis}

With the proposed steady state method, the machine performances are analyzed.

As stated in the research objectives, the main concern in this work is to validate the suggested novel approach for steady state analysis. So different terminal connections and load conditions are considered, but no optimal capacitance combination is examined.

It is observed in the simulation that the solution is, in some extent, sensitive to the leakage inductances and inductance in q axis $L_{m q}$. For example, under the pure resistive load condition, when the load resistance is small, the solved inductance $L_{m d 1}$ can be a complex number, which means the given inductances are not accurate. This may come from several sources. One is the inaccurate leakage inductance and $L_{m q}$, another source is because the nonlinear saturation effect to $L_{m q}$ is neglected. Especially, the later source will make the theoretical performance for low resistance load hard to predict. But in general, operation for the reluctance generator always sticks on the period $\mathrm{AB}$, which is shown on Figure 6-24, to obtain higher output voltage. So estimation for the really low resistance load at BC part is only of limited interest. 


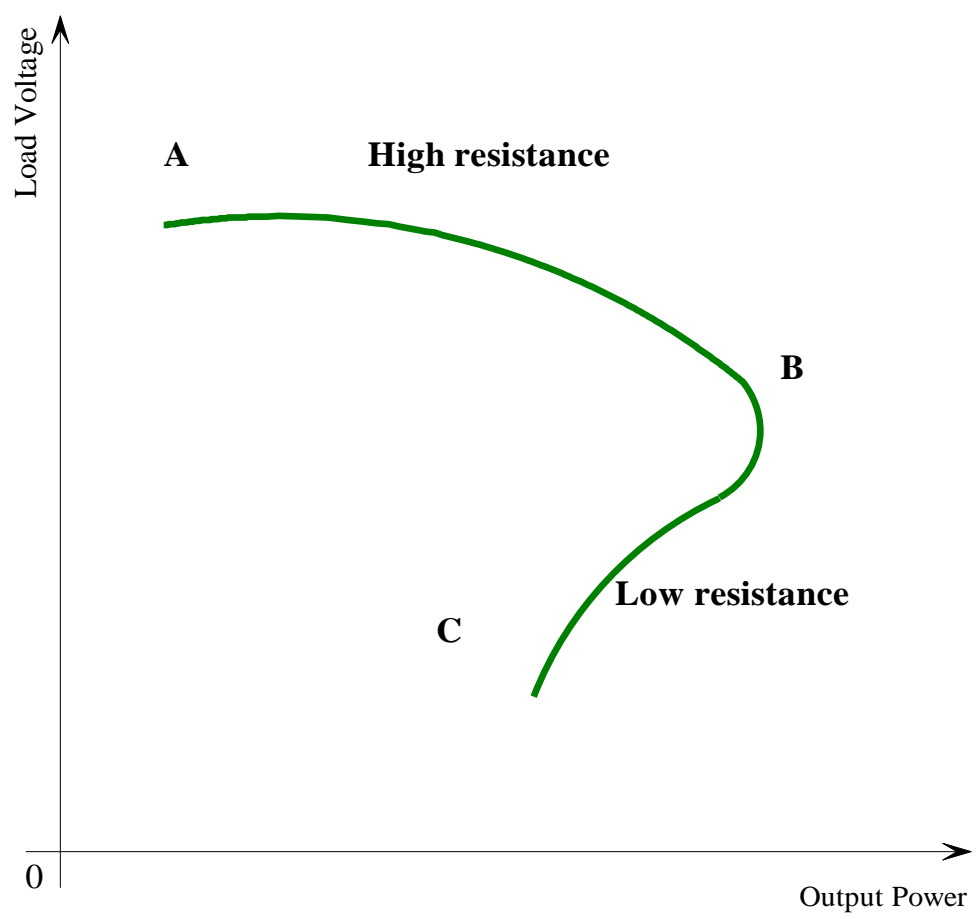

Figure 6-24: Load output vs output power

To increase the accuracy of the steady state analysis, either an accurate measurement for the given inductances has to be applied, or the saturated curve for the $L_{m q}$ must be considered.

\subsubsection{Resistive Load}

A comprehensive comparison is given between simulation and experimental results for pure resistive load. Figure 6-25 through Figure 6-28 represent the case that $C_{m}=127 \mu F, C_{a}=267 \mu F, \omega_{r}=188.5 \mathrm{rad} / \mathrm{s}$. Star points represents the measurement

points, the dotted line is the data fitting curve for the experimental data, while the solid line is the simulated curve. 


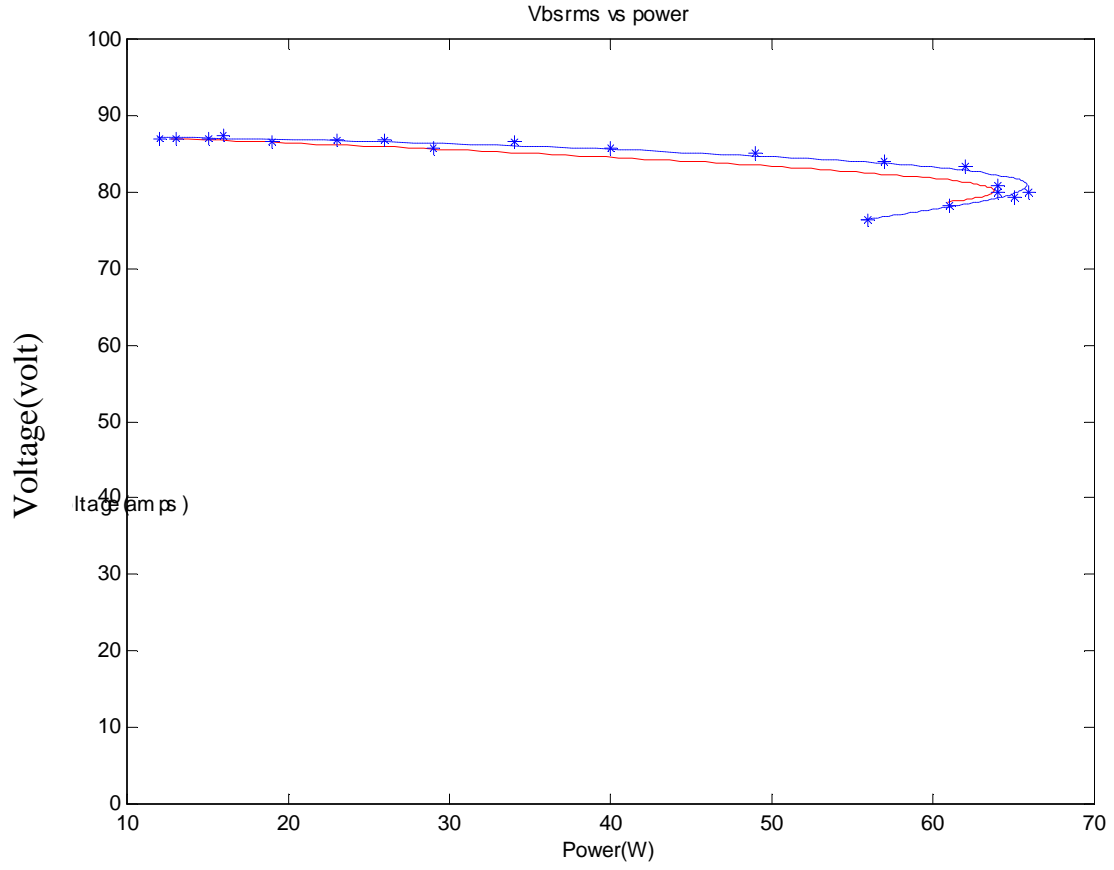

Figure 6-25: Auxiliary winding output voltage vs output power (Dotted-experiment result, Solid—simulation)

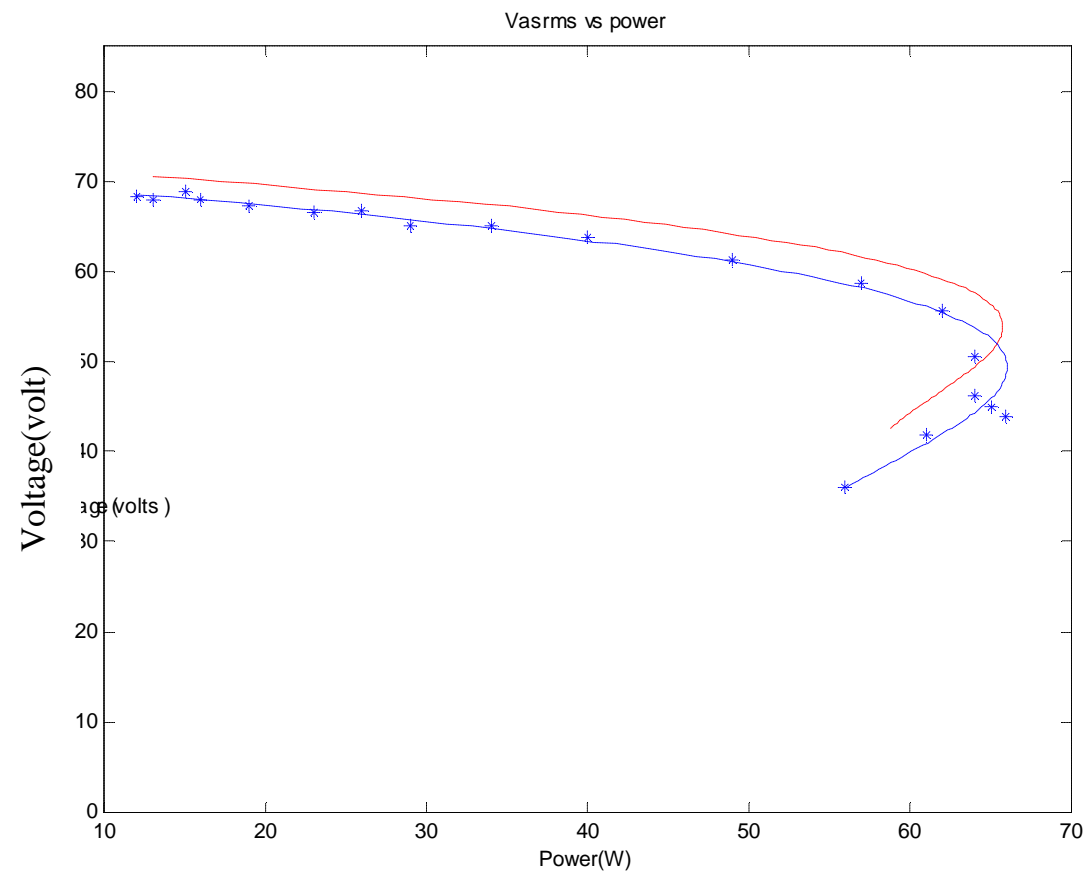

Figure 6-26: Main winding output voltage vs output power (Dotted—experiment result, Solid—simulation) 


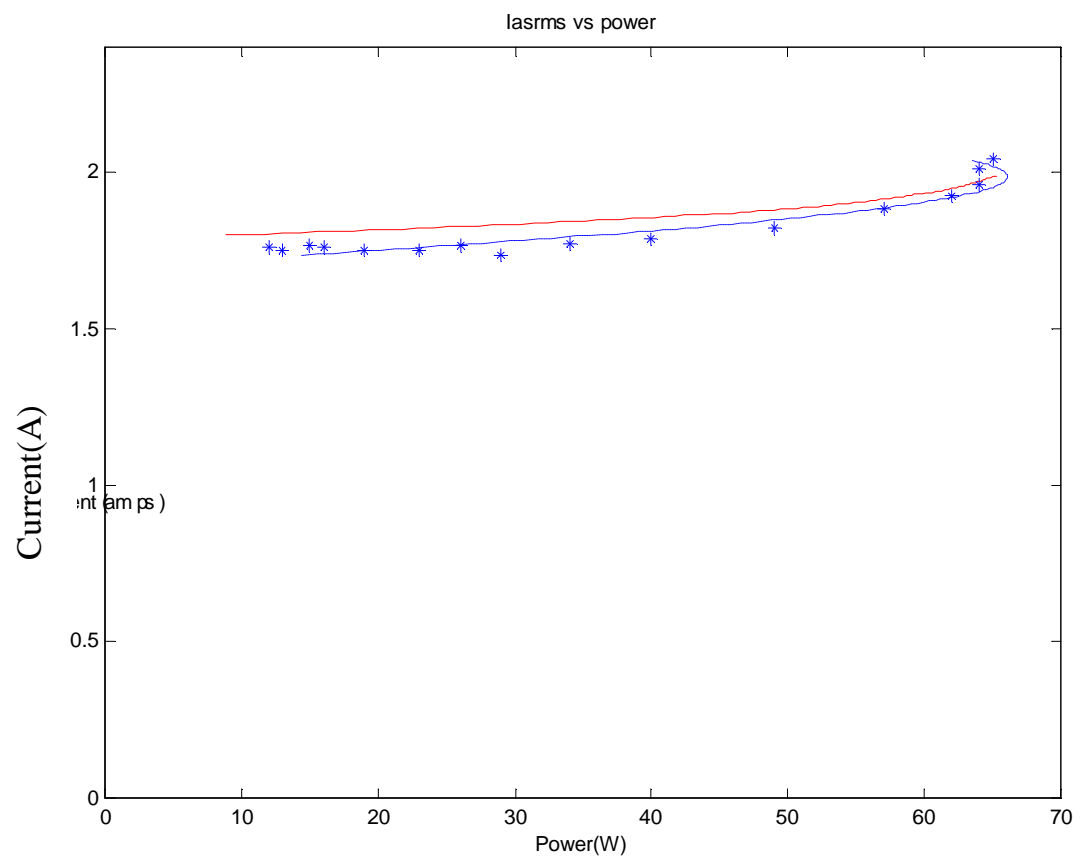

Figure 6-27: Main winding current vs output power

(Dotted—experiment result, Solid—simulation)

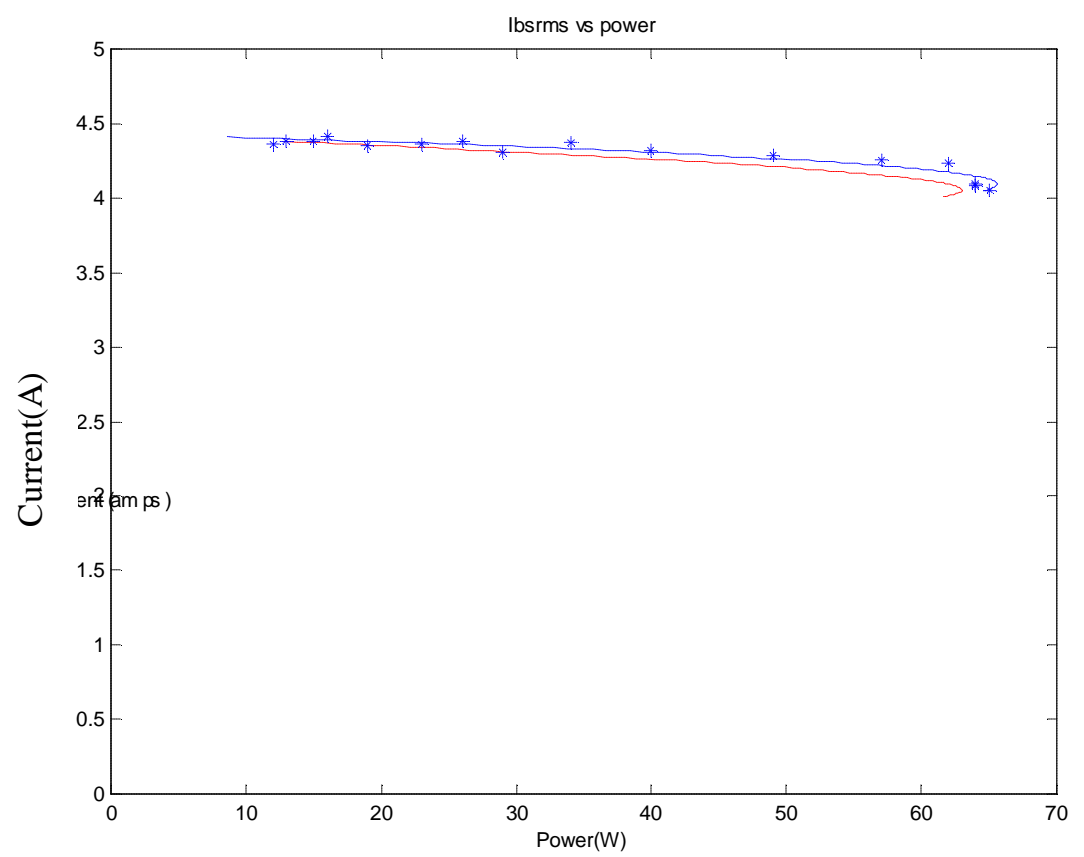

Figure 6-28: Auxiliary winding current vs output power (Star points-experiment result, Solid—simulation) 
Figure 6-29 shows the simulated flux vs resistance relationship under two conditions. One is $C_{m}=127 \mu \mathrm{F}, C_{a}=267 \mu \mathrm{F}$ and $\omega_{r}=188.5 \mathrm{rad} / \mathrm{s}$. the other is $C_{m}=98 \mu F, C_{a}=267 \mu F, \omega_{r}=188.5 \mathrm{rad} / \mathrm{s}$.

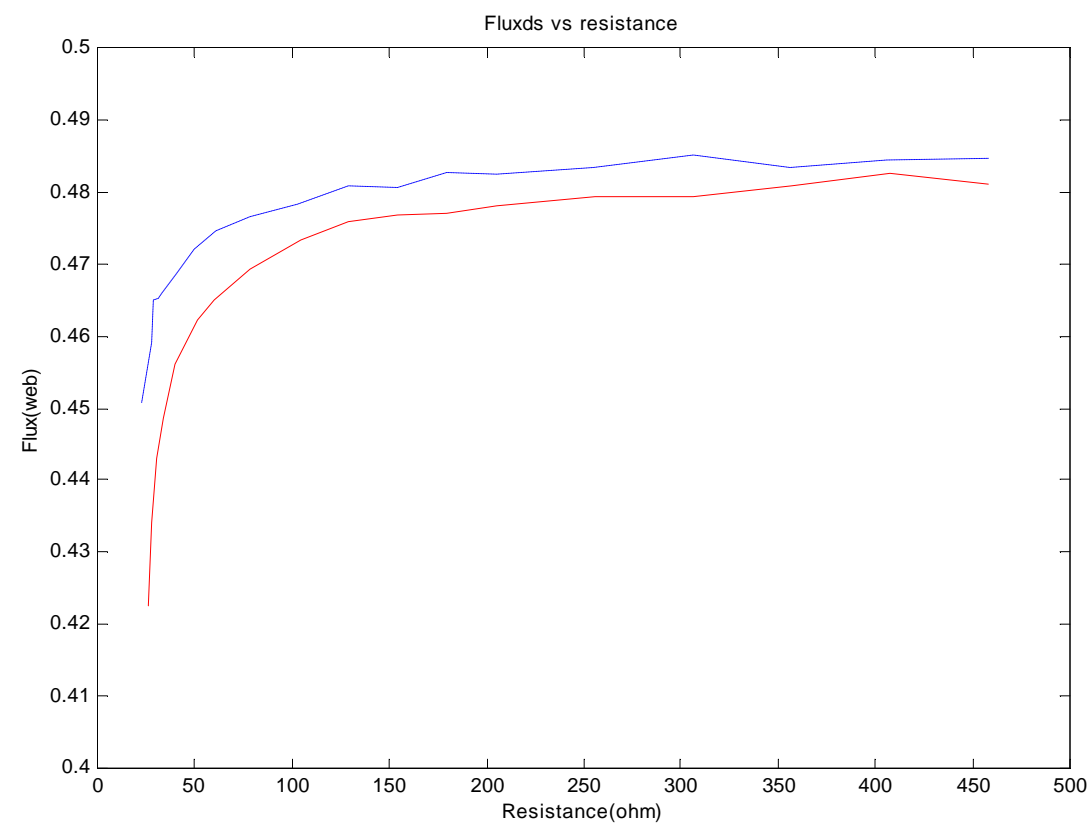

Figure 6-29: Airgap flux vs resistance

(Dotted-- $C_{m}=127 \mu F, C_{a}=267 \mu F ;$ solid-- $\left.C_{m}=98 \mu F, C_{a}=267 \mu F\right)$

From these comparisons, it is apparent that the proposed method for steady state analysis can describe system performance in good agreement. However, in both cases, the main winding terminal voltage and current are overestimated and those of the auxiliary winding are underestimated. The detailed analysis for the deficiency is given in last section.

\subsubsection{RL Load}


When the loads are resistance and inductance, algorithm proposed in chapter 5 is used to obtain the predicted performance. Figure 6-30 through 6-33 show the steady state characteristics with $C_{m}=127 \mu \mathrm{F}, C_{a}=267 \mu \mathrm{F}, \omega_{r}=188.5 \mathrm{rad} / \mathrm{s}$ and $L_{m}=133 \mathrm{mH}$. The performance can be simulated in acceptable accuracy.

It is also observed from the experiment that even though the output voltage is lower than in the case of resistive load under the same output power, the machine can run more stably at low resistance under RL load than pure resistive load. The reason is that the energy stored in the inductance can help the machine extend its stable operation region.

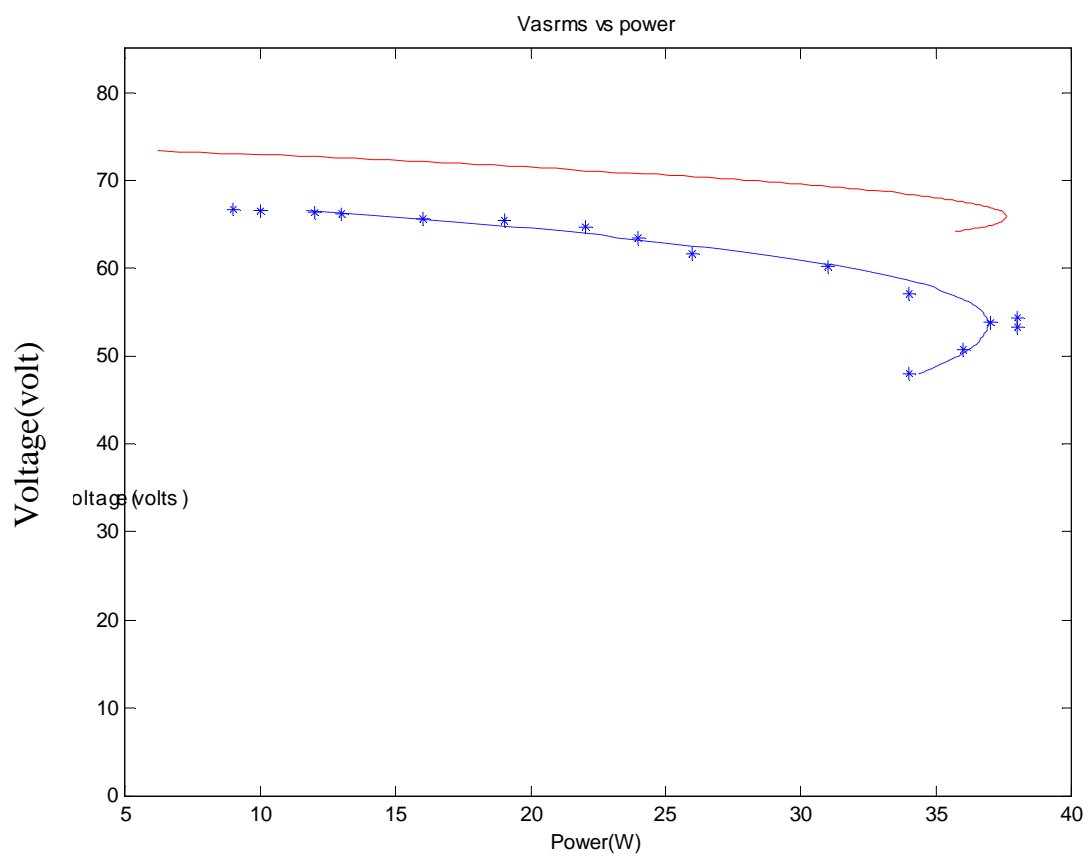

Figure 6-30: Main winding terminal voltage vs output power (Dotted—experiment result, Solid—simulation) 


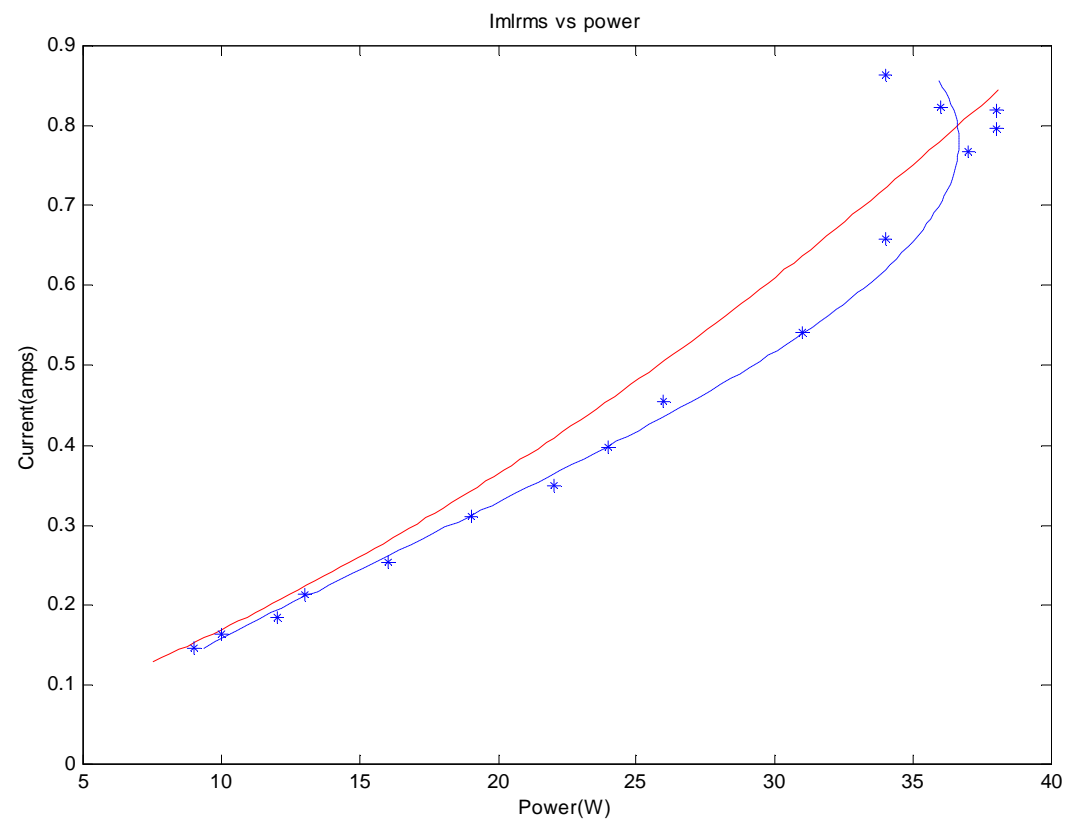

Figure 6-31: Load current vs output power

(Dotted-experiment result, Solid—simulation)

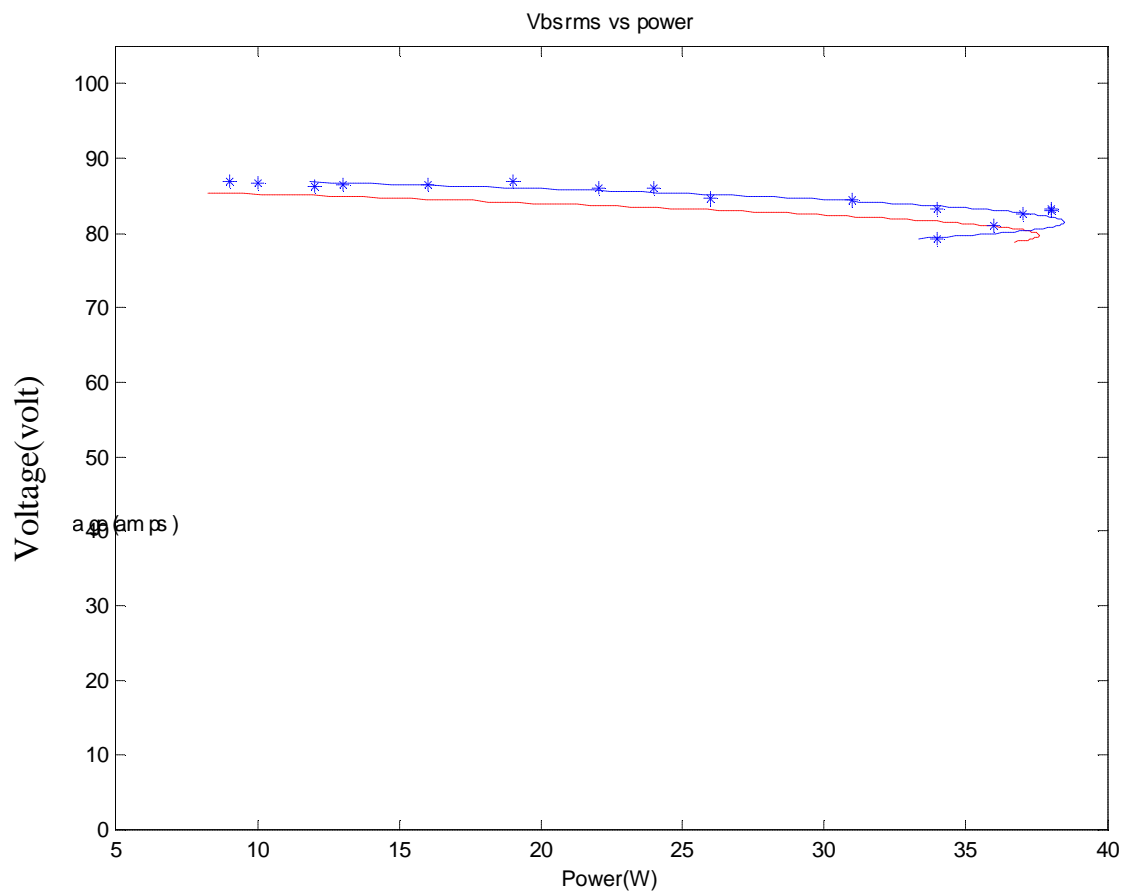

Figure 6-32: Auxiliary winding terminal voltage vs output power (Dotted-experiment result, Solid—simulation) 


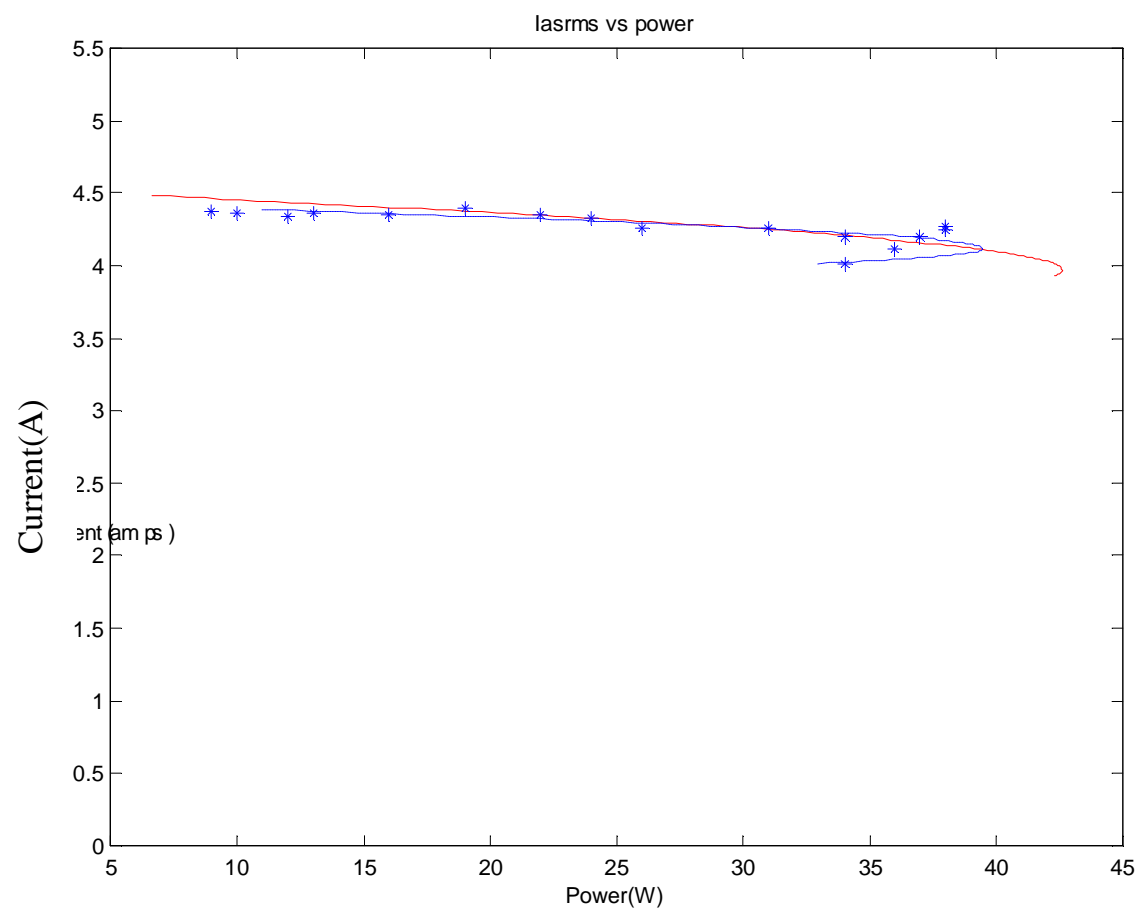

Figure 6-33: Auxiliary winding current vs output power (Dotted-experiment result, Solid—simulation)

\subsubsection{Error Sources for Steady State Analysis}

From the above analyses, the proposed model can reasonably predict the steady state performance. But there are discrepancies. The factors, which can effect the accuracy of the method, are:

(1): Neglecting high order harmonic

When the harmonic balance technique is used in this problem, only DC component and second order component are considered. All the rest of high order harmonics are neglected, which, even though simplify the analysis, bring error to the result. Especially under heavy load, the outputs are distorted more seriously than under 
light load. So when the terminal capacitance is small, the algorithm sometimes cannot find a real inductance value.

Moreover, in the proposed method, DC and AC components of the inductance in d-axis are solution of third and second order polynomials. Neglecting high order harmonics may make the solved inductances deviate from the actual values, which, as a result, can cause the relevant state variables overestimated or underestimated.

(2): Inductance in the quadratic axis

In this method, the inductance in q-axis $L_{m q}$ is assumed constant, which means no saturation in the quadratic direction. But the inductance in direct axis $L_{m d}$ solved by this method is sensitive to $L_{m q}$. Therefore, error can arise because of the inaccurate $L_{m q}$. To get ride of this problem, an iterative process is used. The process is shown in Figure 6-33.

But this method cannot work for all terminal capacitances. Especially under heavy load, the iterative process may get unreasonable inductance values.

\section{(3): Assumption for inductance}

In the proposed method, the inductance for the direct axis is simply defined as $L_{d s}=L_{d s 1} \cos \left(\theta_{1}\right)+L_{d s 3}$.

Satisfying the self-excitation requirement and basing on harmonic balance technique, $L_{d s 3}$ and $L_{d s 1}$ can be solved. After that, the DC component of flux can be obtained by $\lambda_{3}=f_{3}\left(L_{d s 3}\right)$. But the AC component has to be solved from $\lambda_{1}$ and $\lambda_{2}$, as shown in Figure 6-34. Even though because of the nonlinear relationship between inductance and flux, the AC inductance $L_{d s 1} \cos \left(\theta_{1}\right)$ can cause different flux profiles in first and second half period, the flux can still be described as sinusoidal functions. 


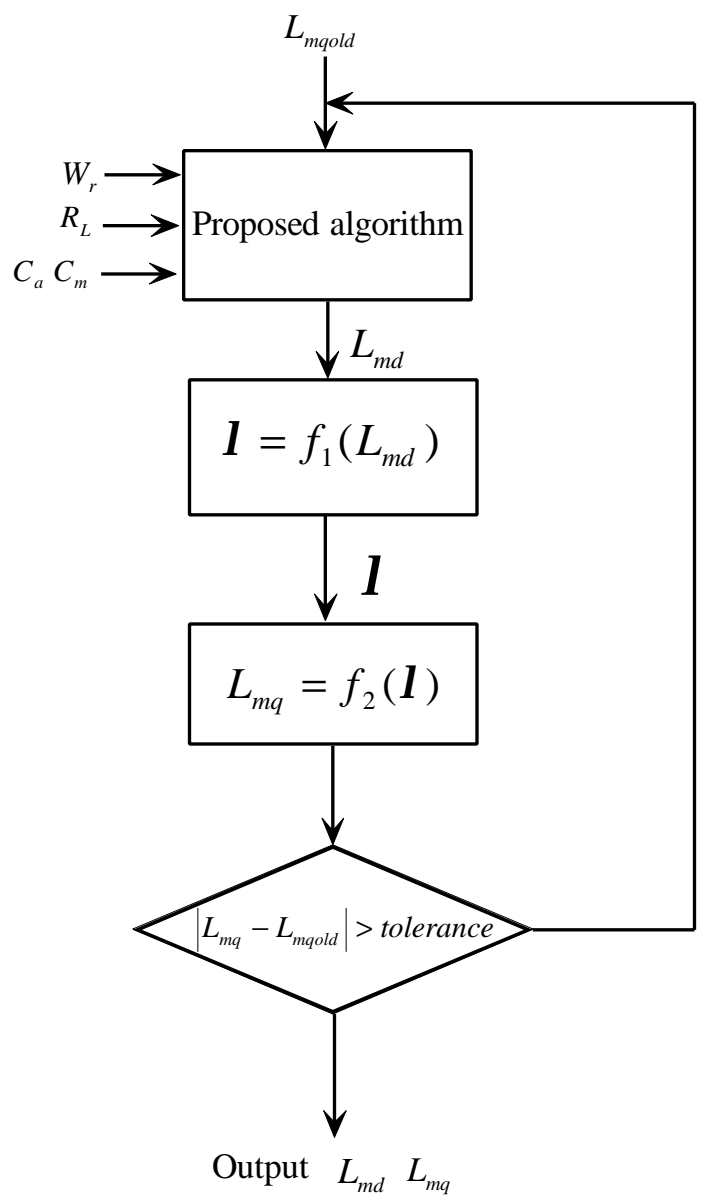

Figure 6-33: Flow chart of iterative process for solving inductance

However, from the transient simulation results shown in Figure 6-35, the AC component of the flux is not a sinusoidal waveform, therefore, the relevant $\mathrm{AC}$ inductance cannot be a sinusoidal waveform, which indicates that because of this simplified assumption, certain error can be induced. 


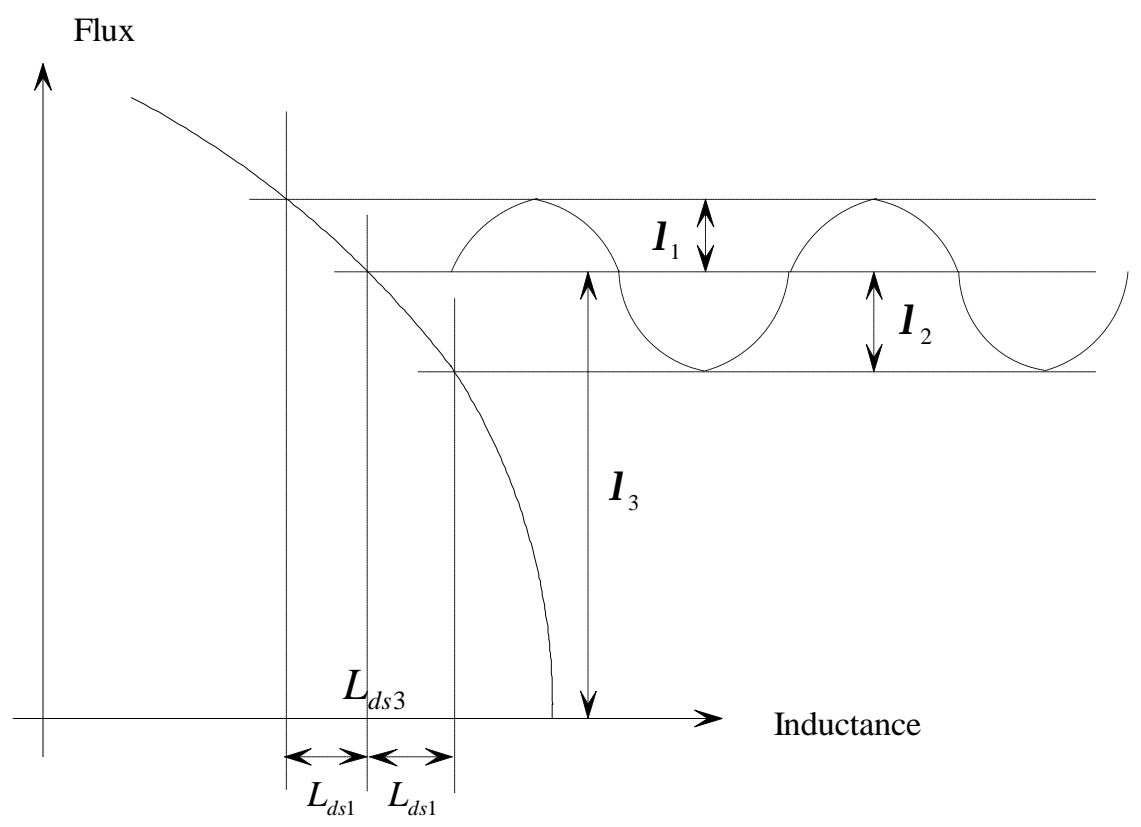

Figure 6-34: Saturation effect to the flux

(4): Flux in q-axis

It is assumed that all the flux is aligned with the d-axis, which is accurate enough for the definition of the saturation curve. But after the inductances are obtained and algorithm goes to the step for solving the current and voltage, the fluxes in q axis have to be considered. As shown in Figure 6-40, the AC component of the q-flux has almost the same amplitude with the AC component of the d-flux and relationship of flux in phasor form leads 90 degree in phase. So in this proposed method, $\lambda_{q s a c}=\lambda_{d s a c} \angle 90^{\circ}$. But both simulation and experimental results show that their amplitudes may deviate with different load and different terminal capacitances, which may generate error. 


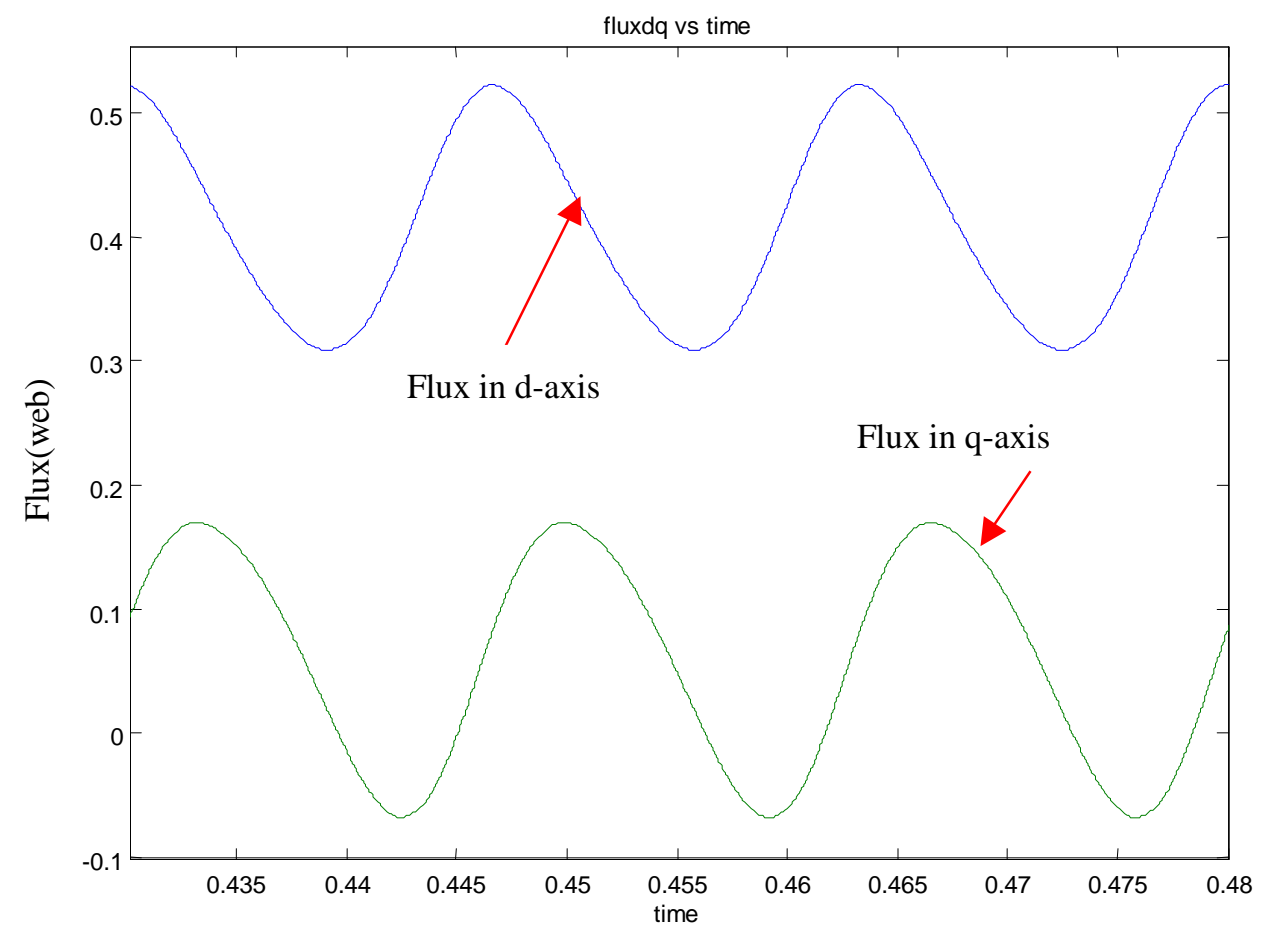

Figure 6-35: $\mathrm{q}$ and $\mathrm{d}$ flux vs time

Moreover, there is also a DC component in the q flux, which is neglected in this method.

Because of the above error sources, the relative error of the simulation can be as high as $12 \%$. 


\section{CHAPTER}

\section{SEVEN}

\section{CONCLUSION}

In this work, the primary objective is to provide a methodology to solve the transient response and steady state performance for single-phase self-excited reluctance machines considering their nonlinear characteristics and unbalance caused by loads. To accomplish this goal, an off-line FEM+SS method is proposed to obtain the nonlinear characteristics of the system, and can be applied to both transient and steady state analyses. Meanwhile, an inductance oriented approach integrated with the harmonic balance technique is introduced to predict the steady state performance and reveal the parametric dependence, which can provide guidance for SPSERG design.

\section{$\underline{\text { 7.1 System Modeling and Simulation for Transient Analysis }}$}

To analyze the transient response, a mathematical model is developed in Chapter 2. With the measured saturation curves, the proposed model can accurately model the transient.

As the requirement of a good design environment, the FEM method is introduced to get rid of the necessity of producing a prototype. With the integration of the material 
nonlinearity to the finite element model, the nonlinear inductance, which is the key parameter reflecting the machine nonlinear characteristics, can be solved based on energy perturbation technique. Even though inductances obtained by this approach can be very accurate, it is difficult to find a definite analytic representation for the relationship between flux and inductance. As described in Chapter 4, there is more than one inductance corresponding to one flux and it is impossible to provide the judgment to take one distinct inductance as the appropriate value.

There are two solutions for this problem. One is to directly integrate the inductance into the state space model using the stator currents as the state variables, and use an iterative process to estimate the actual inductance and the state variables. However, this method is computationally intensive and cannot be implemented on less than a mainframe computer. The second solution is to represent the computed instantaneous inductance as average inductances. Just as in the experimental inductance measuring process, an $\mathrm{AC}$ current is injected into the main winding and the resulting instantaneous inductances $L_{m d}$ and $L_{m q}$ are solved for one cycle, then the average inductances are obtained. With the change in amplitude of the AC current, the saturation curves can finally be attained, which are monotonous and can be easily depicted in analytic expression.

Using the computed saturation curves, the system transient response can be predicted. Furthermore, the saturation curves can also be used in steady state analysis, which provide a complete numerical design environment. 
Simulation and experimental results are compared to illustrate the effectiveness of the transient model.

\section{$\underline{7.2 \text { Steady State Model Development and Simulation }}$}

From machine design and control points of view, a parametric study is preferable to reveal the dependence of system parameters such as load impedance and terminal capacitance on self-excitation and output voltage. So a separate steady state analysis is necessary to depict not only the output waveform but also the steady state performance.

Because of the unbalance nature of the system, some assumptions, such as fictitious circuit, have to be given for SPSERG's QD model, which is suitable for its steady state analysis. A pure resistive load and an RL load are taken into account to move the problem from a special case to a general model.

Next, the harmonic balance technique is applied to the QD model in conjunction with the autonomous feature of the system to build a constraint for all the parameters. The last step is to determine the dependence of the machine parameters embedded in the

constraint $|\bar{M}|=0$ as shown in Chapter 5. By giving terminal capacitance, load and rotor speed, the nonlinear inductance can be solved for using the proposed algorithm. Then based on the nonlinear relationship between inductance and flux $\lambda=f(L)$, the relevant flux values are solved for. Finally, using the relationship between flux and current, current and voltage, all the machine variables are finally determined. 
The uniqueness of this approach is that unlike the traditional application of the

flux and inductance relationship as $L=f(\lambda)$, an inverse relationship $\lambda=f^{-1}(L)$ is used, which is very suitable for unbalanced load condition.

The validation of the proposed method is proven by the comparison between the experimental and the simulation results.

\section{$\underline{7.3 \text { Future Work }}$}

For the transient analysis, the high computation effort prevents the application of the time stepping FEM+SS approach. But with the advancement of the computer technology, the computational power and speed of the processor are increasing exponentially. Furthermore, software packages for finite element analysis are becoming more professional and more flexible in multiphysics. It can be expected that electromagnetic field will be directly coupled with the circuit model, which can in turn make the package more efficient and applicable on a PC workstation. Therefore, in the near future, a complete design environment can be established with limited computation requirement.

In the steady state analysis, only certain harmonics are considered in this study. As a validation for the methodology, it is successful and provides good insight into the parameter dependencies. However, if a more general model is considered, which includes higher order harmonics in the harmonic balance technique, then a more accurate solution can be expected. 
Moreover, the proposed algorithm in steady state analysis is sensitive to the leakage inductance, which in nature has 3-D configuration. So 3-D field analysis will certainly be helpful for the accurate estimation of the leakage inductance.

In this study, only machine modeling and design are considered. However, stable operation of this kind of self-excited generator is always a problem, for it depends not only on the rotor speed and terminal capacitance, but also the load. So after a good understanding of the machine characteristics, control scheme has to be applied to obtain a more stable operation. And the results form the nonlinear study in this work will be very essential for the control system stability analysis. 


\section{Bibliography}

[ $\left.{ }^{1}\right]$ ]: I. Boldea, Z.X.Fu, S.A.Nasar, “ High-performance Reluctance Generator”, IEE proceedings, Part B: Electric Power Applications v 140 n 2 Mar 1993 pp124-130

$\left.{ }^{2}\right]$ : Y.H.A.Rahim, A.L.Mohamadien, " Comparison between the Steady-state Performance of Self-excited Reluctance and Induction Generators", IEEE Transactions on Energy Conversion, vol 5, No. 3, September 1990, pp519-525

[3]: A.L.Mohamadein, Y.H.A.Rahim, A.S.Al-Khalaf, “ Sreadt-state Performance of Selfexcited Reluctance Generators”, IEE Proceedings part B, vol 137, No. 5, September 1990

[ $\left.{ }^{4}\right]$ : Muljadi, E.; Lipo, T.A., " Series compensated PWM inverter with battery supply applied to an isolated induction generator", IEEE Transactions on Inductry Applications, vol 30, No. 4, July/August 1994.

$\left.{ }^{5}\right]$ : P. C. Krause, O. Wasynczuk, S. Sudhoff, “ Analysis of Electric Machinery”, IEEE Press, 1995, ISBN 0-7803-1101-9

[ $\left.{ }^{6}\right]$ : R.J.Kerman, “ Machine Anakysis with UnbalanceTerminal Constraints by d-q Harmonic Balance", IEE Proceedings, vol 128, Prt B, No 6, November 1981

[7]: Radim, Y.H.A., Alyan, A.A.S., “Effect of Excitation Capacitors on Transient Performance of Reluctance Generators", IEEE Transactions on Energy Conversion v $6 \mathrm{n}$ 4 Dec 1991, pp 714-720

$\left[{ }^{8}\right]$ : Olorunfemi Ojo, “ Limit-Cycle and Small-Signal Dynamics of Self-Excited Synchronous Reluctance Generator", IEEE Proceeding of Southeastern Symposium on System Theory, $26^{\text {th }}$ MAR 1994, pp244-248

[9]: Olorunfemi Ojo, Antonio Ginart, O.Omozusi, A.A. Jimoh, “ Modeling and Analysis of A Single-Phase Synchronous Reluctance Machine Including Saturation Effect", IEEE Industry Application Society Annual Meeting, New Orleans, Louisiana, October 5-9, 1997.

$\left[{ }^{10}\right]$ : T.F.Chan, " Steady-State Analysis of a Three-Phase Self-Excited Reluctance Generator”, IEEE Trans. Energy Conversion, Vol.7, No.1, March 1992.

$\left[{ }^{11}\right]$ : Li Wang, Yung-Shan Wang, "Dynamic Performance and Minimum Loading Effects of An Isolated Self-Excited Reluctance Generator", Proceedings of the 1999 Winter Meeting of IEEE Power Engineering Society. Part 1 (of 2) Jan 31-Feb 41999 v 1 New York, NY, p 13-17003216 
$\left[{ }^{12}\right]$ : Al-Bahrani, A.H.; Malik, N.H., " Steady state analysis and performance characteristics of a three phase induction generator self excited with a single capacitor", IEEE Transactions on Energy Conversion v5 n4 Dec. 1990 p725-732

[13]: Li Wang; Ruey-Yong Deng, “ Transient performance of an isolated induction generator under unbalanced excited capacitors", IEEE Transactions on Energy Conversion vol14, No.4, Dec. 1999, p887-893

$\left[{ }^{14}\right]$ : Smith, A.C., Williamson, Stephen, Smith, J.R., " Transient Currents and Torque in Wound-Rotor Induction Motors Using the Finite Element Method", IEE Proceedings, part B: Electric Power Applications v137 n3 May 1990 p160-173

$\left[{ }^{15}\right]$ : Antonio C. Ferreira, Stephen Williamson, “ Time-Stepping Finite-Element Analysis of Brushless Doubly Fed Machine Taking Iron Loss and Saturation into Account", IEEE Transactions on Industry Application,vl. 35, No.3, May/June 1999, p583-588

$\left[{ }^{16}\right]$ : Williamson, Stephen, Flack, Timothy J., Volschenk, Albertus F., " Representation of Skew in Time-stepped Two-Dimensional Finite Element Models of Electrical Machines", IEEE Transactions on Industry Applications v31 n5 Sep-Oct 1995, p1009-1015

[ $\left.{ }^{17}\right]$ : Cingoski, Vlatko; Mikami, Mitsuru; Yamashita, Hideo; Inoue, Kenji, “Computer Simulation of a Three-Phase Brushless Self-Excited Synchronous Generator", IEEE Transactions on Magnetics, Proceedings of the $199818^{\text {th }}$ Biennial IEEE Conference on Electromagnetic Field Computation Jun 1-3 1998 v35 n3 May 1999, Tucson, AZ, USA

$\left[{ }^{18}\right]$ : Deng F., N.A.O. Demerdash, " A Coupled Finite-Element State-Space Approach for Synchronous Generators. Part I: Model Development", IEEE Trans. Aerospace and Electronic Systems, vol. 32, No.2, April 1996, pp775-784.

$\left[{ }^{19}\right]$ : Deng F., N.A.O. Demerdash, “ A Coupled Finite-Element State-Space Approach for Synchronous Generators. Part II: Applications", IEEE Trans. Aerospace and Electronic Systems, vol. 32, No.2, April 1996, pp785-794.

[20]: Deng F., Demerdash, N.A., " CFE-SS approach for salient-pole synchronous generators under unbalances", IEEE Tran. Aerospace and Electronic Systems, v33n 1Jan 1997, pp142-151.

$\left[{ }^{21}\right]$ : Deng F., Demerdash, N.A., " Part II: Balanced and Unbalanced Rectifier Loads ", IEEE Trans. Aerospace and Electronic Systems, v33n 1Jan 1997,pp152-162

[22]: N.A. Demerdash, P. Baldassari, “ A Combined Finite Element-Sate Soace Modeling Environment for Induction Motors in the ABC Frame of Reference: The No-Load Condition”, IEEE Transactions on Energy Conversion, Vol. 7, N0. 4, Dec. 1992 pp698709.

$\left[{ }^{23}\right]$ : Bulent Sarlioglu and Thomas A. Lipo, "Nonlinear Modeling and Simulation of Single Phase Doubly Salient Perment Magnet Generator", Proceedings of the 1998 IEEE 
Industry Applications Conference. Part 1 (of 3) Oct 12-15 1998 v1 1998 St.Louis, MO, USA p 18-26 0197-2618

[24]: T. W. Nehl, F. A. Fouad, N. A. Demerdash, "Determination of Saturated Values of Rotating Machinery Incremtntal and Apparent Inductance by an Energy Perturbation Method", IEEE Transactions on Power Apparatus and Systems, vol. PAS-101,No.12, Dec. 1982

$\left[{ }^{25}\right]$ : Kundert, Kenneth S., Sangiovanni-Vincentelli, Alberto, “ Simulation of Nonlinear Circuits in the Frequency Domain", IEEE Transactions on Computer-Aided Design of Integrated Circuits and Systems v CDA-5 n4 Oct 1986, pp521-535

$\left[{ }^{26}\right]$ : Kundert, Kenneth S., Sorkin, Gregory B., Sangiovanni-Vincentelli, Alberto, “ Applying Harmonic Balance to Almost-periodic Circuit", IEEE Transactions on Microwave Theory and Techniques v36 n2 Feb. 1988 pp366-378 


\section{APENDIX I: Program Code}

\section{steadystateR.m}

\% This program is the main function obtaining the steady state

\% performance of the single-phase self-excited reluctance generator

\% with pure resistance load.

\% Related file: funssi.m

close all;

clear all;

Lmq $=0.02600$;

Lenbdaqs $3=0.05$;

- Main winding terminal capacitance

$\mathrm{Cm}=125 e-6$;

ㅇ Auxiliary winding terminal capacitance

$\mathrm{Ca}=267 e-6$;

\% load resistance vector

Rout $=[458 ; 407 ; 357 ; 306 ; 256 ; 205 ; 179 ; 154 ; 129 ; 104 ; 78 ; 60 ; 50 ; 40 ; 30 ; 20]$;

L=length (Rout11);

funss1 (Cm, Ca, Rout, L, Lmq, Lenbdaqs3);

\section{funss1.m}

\% This function deals with the steady state performance for the

\% reluctance generator under pure resistance load

\% The idea is that given load resistance, terminal capacitance and

o rotor speed, this approach determines the saturated inductance in $d$

\% axis.

응 First, assume $L_{d s}=L_{d s 1} \cos \left(2 \omega_{r} t\right)+L_{d s 3}$

\% Second, set Lds1=0, and solve $\operatorname{det}(\mathrm{M} 1)=0$ to obtain Lds3

\% Third, take the solved Lds3 back to matrix M1, and solve det (M1)=0 to

obtain Lds1

Fourth, after obtaining $L_{d s 3}$ and $L_{d s 1}$, the relationship fluxds=f(Lds)

is used.

1: fluxqsDC=f $(\operatorname{Lds} 3)$

2: $\quad$ fluxqsAC $=(f(\operatorname{Lds3}-\operatorname{Lds} 1)-f(\operatorname{Lds} 3+\operatorname{Ldfs} 1)) / 2$

$\div$ Fifth, estimate the flux in $q$ axis.

1: fluxqsDC=0.03-0.05;

2: fluxqsAC $=1 * f l u x d s A C$ 


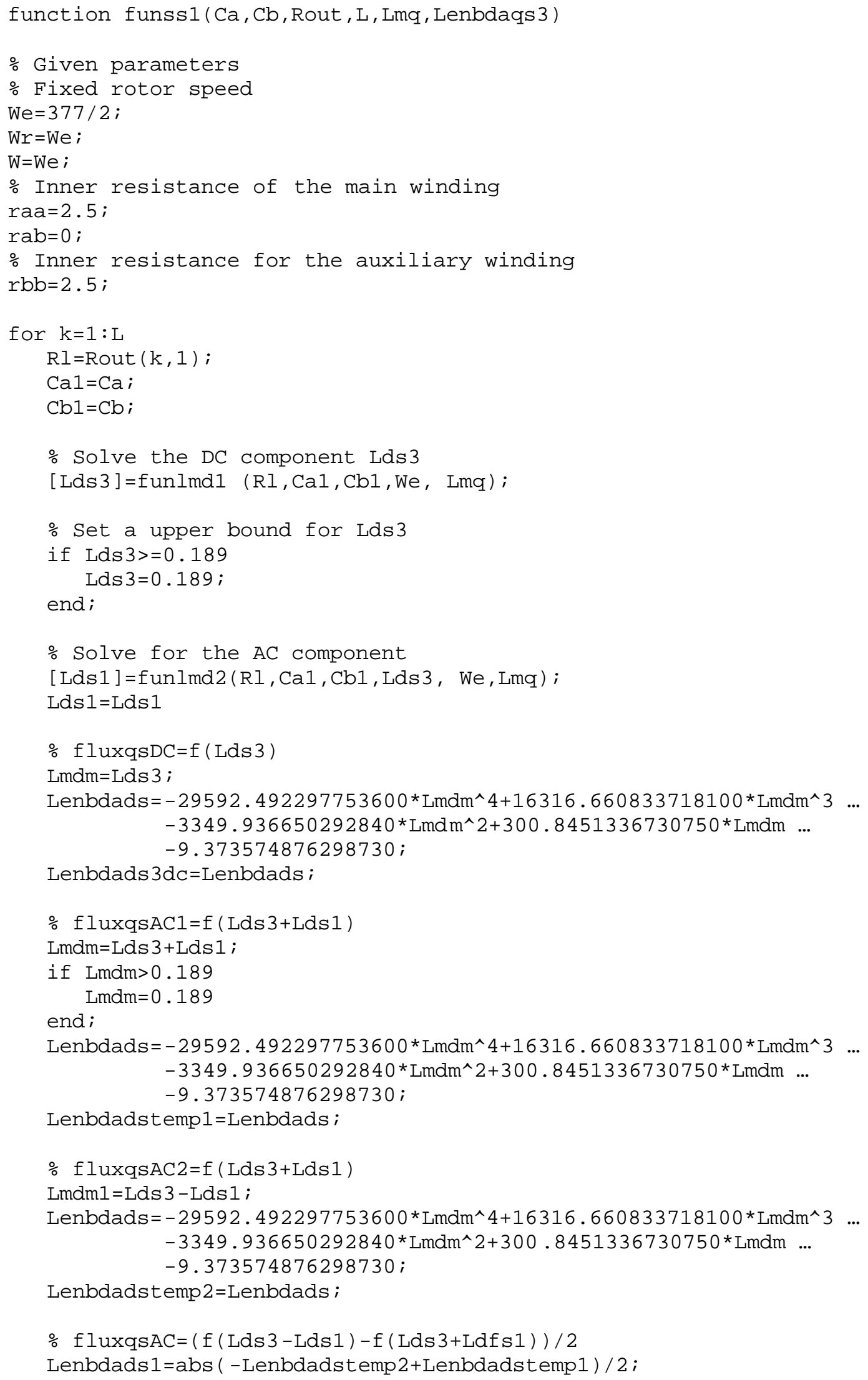




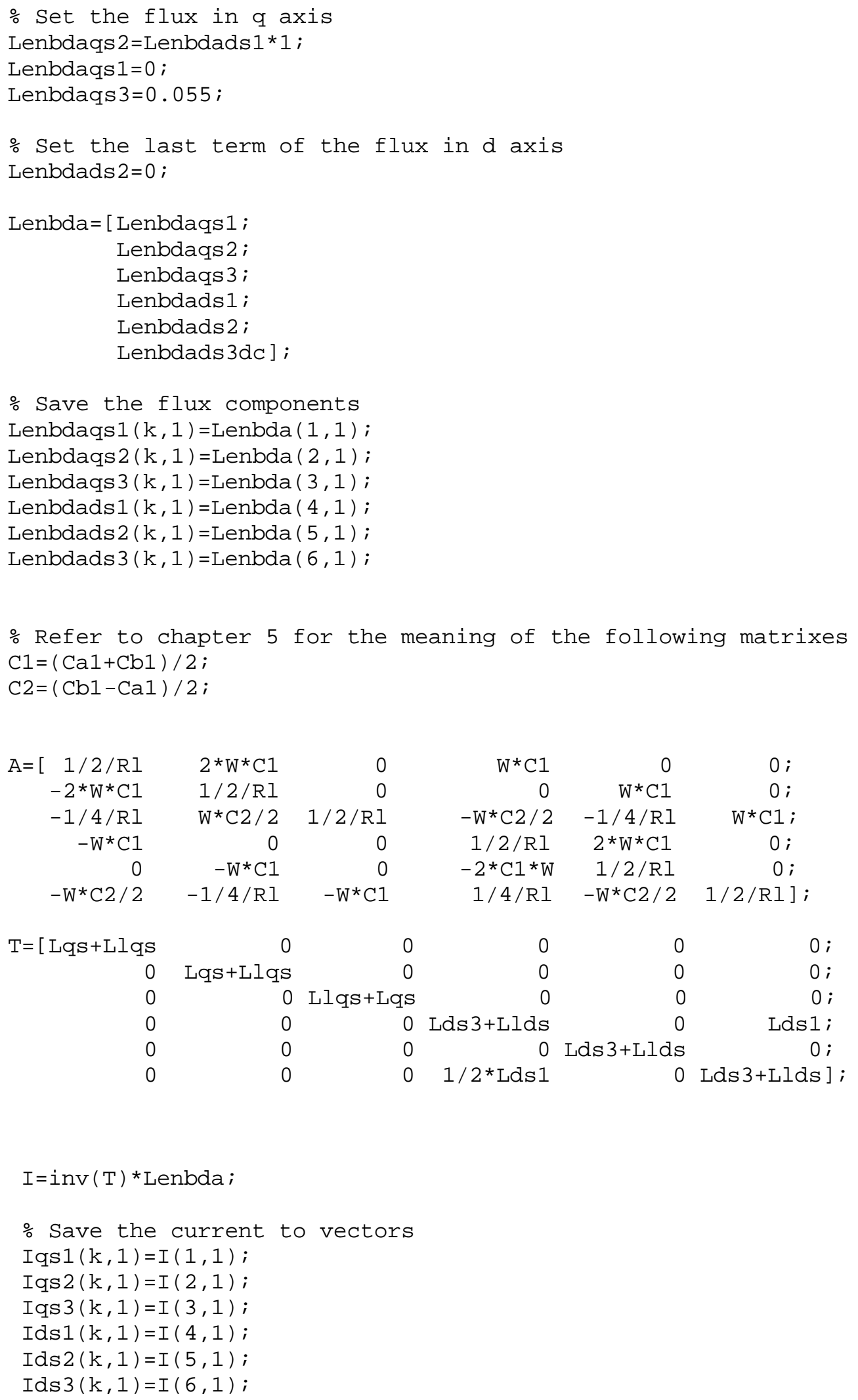




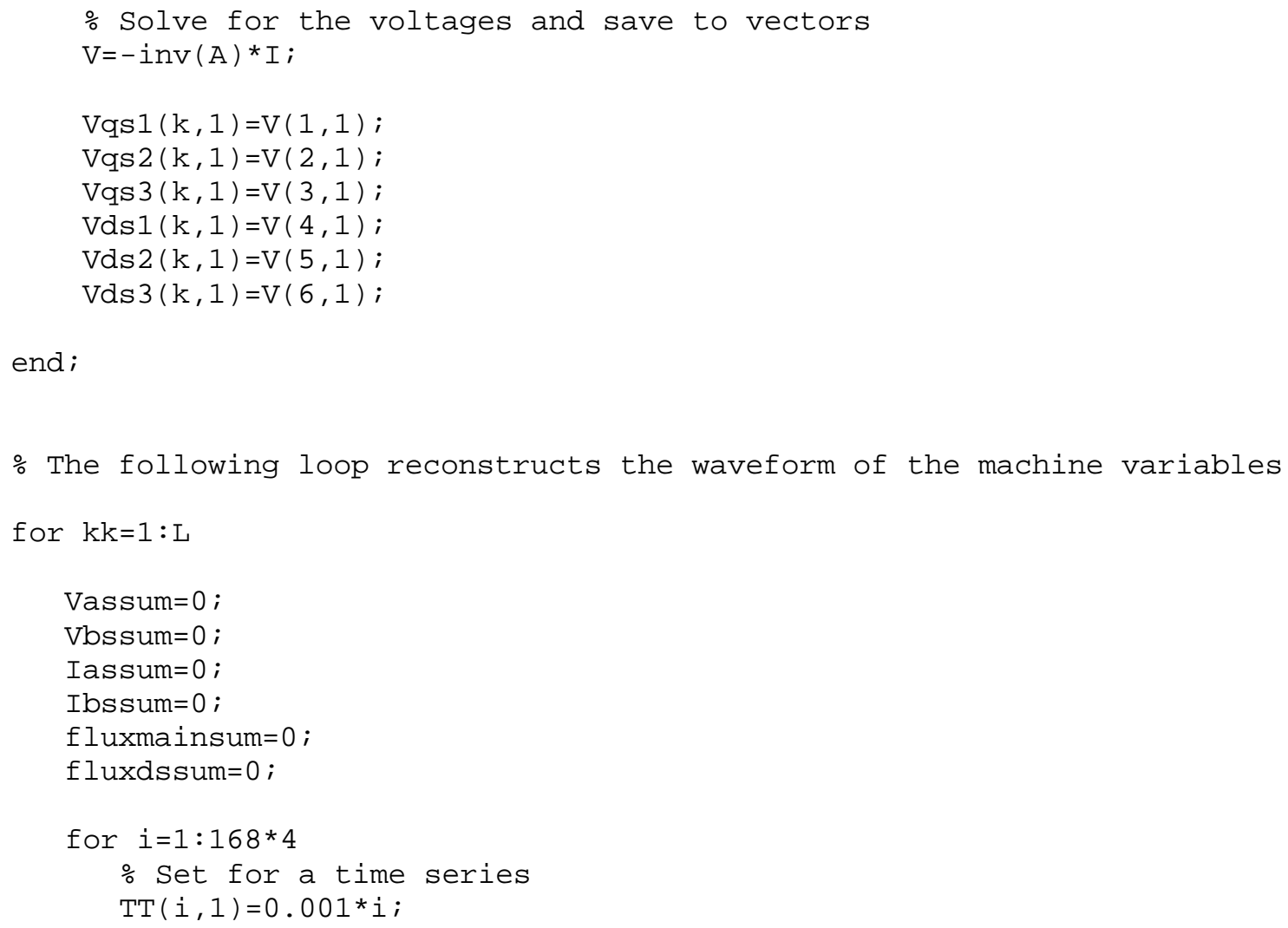

$\%$ Use harmonic balance technique inversely to obtain the voltage \% waveforms in rotor reference frame $\operatorname{Vqs}(\mathrm{i}, 1)=\mathrm{Vqs} 1(\mathrm{kk}, 1) * \cos ((\mathrm{We}+\mathrm{Wr}) * \mathrm{TT}(\mathrm{i}, 1))+\mathrm{Vqs} 2(\mathrm{kk}, 1) * \sin ((\mathrm{We}+\mathrm{Wr})$

*TT(i,1))+Vqs3(kk,1);

$\operatorname{Vds}(\mathrm{i}, 1)=\mathrm{Vds} 1(\mathrm{kk}, 1) * \cos ((\mathrm{We}+\mathrm{Wr}) * \mathrm{TT}(\mathrm{i}, 1))+\mathrm{Vds} 2(\mathrm{kk}, 1) * \sin ((\mathrm{We}+\mathrm{Wr})$

*TT(i,1))+Vds3(kk,1);

$\%$ Apply the transform to get the machine variables $\mathrm{Vm}(\mathrm{Vas})$,

$\% \mathrm{Va}(\mathrm{Vbs})$

$\operatorname{Vbs}(\mathrm{i}, 1)=\operatorname{Vqs}(\mathrm{i}, 1) * \cos (\mathrm{Wr} * \mathrm{TT}(\mathrm{i}, 1))+\operatorname{Vds}(\mathrm{i}, 1) * \sin (\mathrm{Wr} * \mathrm{TT}(\mathrm{i}, 1))$;

$\operatorname{Vas}(\mathrm{i}, 1)=\operatorname{Vqs}(\mathrm{i}, 1) * \sin (\mathrm{Wr} * \mathrm{TT}(\mathrm{i}, 1))-\operatorname{Vds}(\mathrm{i}, 1) * \cos (\mathrm{Wr} * \mathrm{TT}(\mathrm{i}, 1))$;

$\%$ Prepare to take the rms value of the machine variables

Vassum $=\operatorname{Vassum}+\operatorname{Vas}(\mathrm{i}, 1)^{\wedge} 2$;

Vbssum $=\operatorname{Vbssum}+\operatorname{Vbs}(i, 1)^{\wedge} 2$;

$\%$ For currents

$\operatorname{Iqs}(\mathrm{i}, 1)=\operatorname{Iqs} 1(\mathrm{kk}, 1) * \cos ((\mathrm{We}+\mathrm{Wr}) * \mathrm{TT}(\mathrm{i}, 1))+\mathrm{Iqs} 2(\mathrm{kk}, 1) * \sin ((\mathrm{We}+\mathrm{Wr})$

*TT(i,1))+Iqs3(kk,1);

$\operatorname{Ids}(\mathrm{i}, 1)=\operatorname{Ids} 1(\mathrm{kk}, 1) * \cos ((\mathrm{We}+\mathrm{Wr}) * \mathrm{TT}(\mathrm{i}, 1))+\operatorname{Ids} 2(\mathrm{kk}, 1) * \sin ((\mathrm{We}+\mathrm{Wr})$

*TT $(\mathrm{i}, 1))+\operatorname{Ids} 3(\mathrm{kk}, 1)$;

$\operatorname{Ibs}(\mathrm{i}, 1)=-\operatorname{Iqs}(\mathrm{i}, 1) * \cos (\mathrm{Wr} * \mathrm{TT}(\mathrm{i}, 1))-\operatorname{Ids}(\mathrm{i}, 1) * \sin (\mathrm{Wr} * \mathrm{TT}(\mathrm{i}, 1))$;

$\operatorname{Ias}(\mathrm{i}, 1)=-\operatorname{Iqs}(\mathrm{i}, 1) * \sin (\mathrm{Wr} * \mathrm{TT}(\mathrm{i}, 1))+\operatorname{Ids}(\mathrm{i}, 1) * \cos (\mathrm{Wr} * \mathrm{TT}(\mathrm{i}, 1))$;

Iassum=Iassum+Ias $(\mathrm{i}, 1)^{\wedge} 2$;

Ibssum=Ibssum+Ibs $(\mathrm{i}, 1)^{\wedge} 2$;

$\%$ For fluxes 
Fluxqsi $(\mathrm{i}, 1)=$ Lenbdaqs $1(\mathrm{kk}, 1) * \cos ((\mathrm{We}+\mathrm{Wr}) * \mathrm{TT}(\mathrm{i}, 1))+$ Lenbdaqs $2(\mathrm{kk}, 1)$

$* \sin ((\mathrm{We}+\mathrm{Wr}) * \mathrm{TT}(\mathrm{i}, 1))+$ Lenbdaqs3 $3(\mathrm{kk}, 1)$;

Fluxdsi $(\mathrm{i}, 1)=$ Lenbdads $1(\mathrm{kk}, 1) * \cos ((\mathrm{We}+\mathrm{Wr}) * \mathrm{TT}(\mathrm{i}, 1))+\operatorname{Lenbdads} 2(\mathrm{kk}, 1)$

$* \sin ((\mathrm{We}+\mathrm{Wr}) * \mathrm{TT}(\mathrm{i}, 1))+$ Lenbdads3 $(\mathrm{kk}, 1)$;

Fluxms(i,1)=Fluxqsi(i,1)*cos(Wr*TT(i,1))+Fluxdsi(i,1)

$* \sin (\mathrm{Wr} * \mathrm{TT}(\mathrm{i}, 1))$;

Fluxas $(\mathrm{i}, 1)=$ Fluxqsi $(\mathrm{i}, 1) * \sin (\mathrm{Wr} * \mathrm{TT}(\mathrm{i}, 1))$-Fluxdsi $(\mathrm{i}, 1)$

$* \cos (\mathrm{Wr} * \mathrm{TT}(\mathrm{i}, 1))$;

Fluxmain $(i, 1)=\operatorname{sqrt}(\operatorname{Fluxms}(i, 1) \wedge 2+\operatorname{Fluxas}(i, 1) \wedge 2)$;

end;

$\operatorname{Vmsrms}(k \mathrm{k}, 1)=\operatorname{sqrt}(\operatorname{Vassum} / 168 / 4)$;

$\operatorname{Vasrms}(k k, 1)=\operatorname{sqrt}(\operatorname{Vbssum} / 168 / 4)$;

$\operatorname{Imsrms}(k k, 1)=\operatorname{sqrt}(\operatorname{Iassum} / 168 / 4)$;

Iasrms $(k k, 1)=\operatorname{sqrt}($ Ibssum/168/4);

power $(k k, 1)=\operatorname{Vmsrms}(k k, 1)^{\wedge} 2 / \operatorname{Rout}(k k, 1)$; end;

\section{funlmd1.m}

\% This program solves the DC component Lds3 of the inductance in $d$ axis

응

function $[$ Ldsselect $]=f u n l m d 1(\mathrm{Rl}, \mathrm{Ca}, \mathrm{Cb}, \mathrm{W}, \mathrm{Lmq})$

을 Define the variable

Lds $=\operatorname{sym}($ 'Lds', 'real');

\% Given parameters

raa $=2.5$;

$\mathrm{rab}=0$;

$r b b=2.5$;

$\mathrm{C} 1=(\mathrm{Ca}+\mathrm{Cb}) / 2$;

$\mathrm{C} 2=(-\mathrm{Ca}+\mathrm{Cb}) / 2$;

$\mathrm{Wr}=\mathrm{W}$;

$\mathrm{We}=\mathrm{W}$;

$\circ \mathrm{Wr}=\mathrm{We}$;

Lqs $=\mathrm{Llqs}+\mathrm{Lmq}$;

ㅇ Terminal matrix, $I=-\mathrm{AV}$

\begin{tabular}{|c|c|c|c|c|c|c|}
\hline$A=[$ & $1 / 2 / \mathrm{R} 1$ & $2 * \mathrm{~W} * \mathrm{C} 1$ & 0 & $\mathrm{~W}^{\star} \mathrm{C} 1$ & 0 & $0 ;$ \\
\hline & $-2 * \mathrm{~W} * \mathrm{C} 1$ & $1 / 2 / \mathrm{Rl}$ & 0 & 0 & $\mathrm{~W} * \mathrm{C} 1$ & $0 ;$ \\
\hline & $-1 / 4 / \mathrm{R} I$ & $\mathrm{~W} * \mathrm{C} 2 / 2$ & $1 / 2 / \mathrm{R} I$ & $-\mathrm{W} * \mathrm{C} 2 / 2$ & $-1 / 4 / \mathrm{R} 1$ & $\mathrm{~W}^{\star} \mathrm{C} 1 ;$ \\
\hline & $-\mathrm{W}^{\star} \mathrm{C} 1$ & 0 & 0 & $1 / 2 / \mathrm{Rl}$ & $2 * \mathrm{~W} \star \mathrm{C} 1$ & $0 ;$ \\
\hline & 0 & $-W * C 1$ & 0 & $-2 * \mathrm{C} 1 * \mathrm{~W}$ & $1 / 2 / \mathrm{R} I$ & $0 ;$ \\
\hline & $-W^{*} \mathrm{C} 2 / 2$ & $-1 / 4 / \mathrm{R} 1$ & $-\mathrm{W}^{\star} \mathrm{C} 1$ & $1 / 4 / \mathrm{R} 1$ & $-W^{*} \mathrm{C} 2 / 2$ & 1/2/RI]; \\
\hline
\end{tabular}

$\mathrm{AA}=-\operatorname{inv}(\mathrm{A}) ; \circ$ the negative sign indicates that the terminal currents 
\% have the opposite direction with the assumed state

\% current

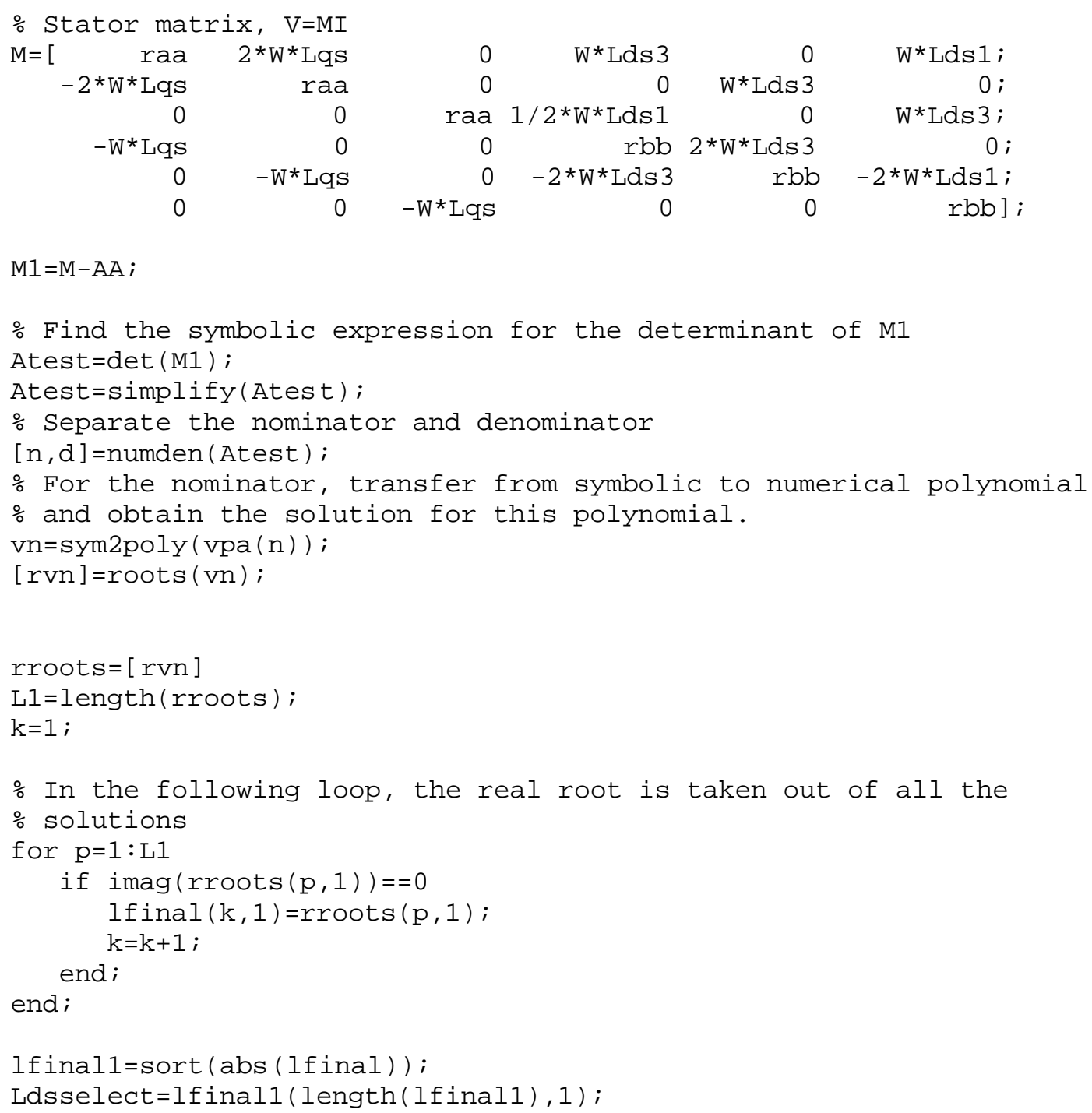

\section{funlmd2.m}

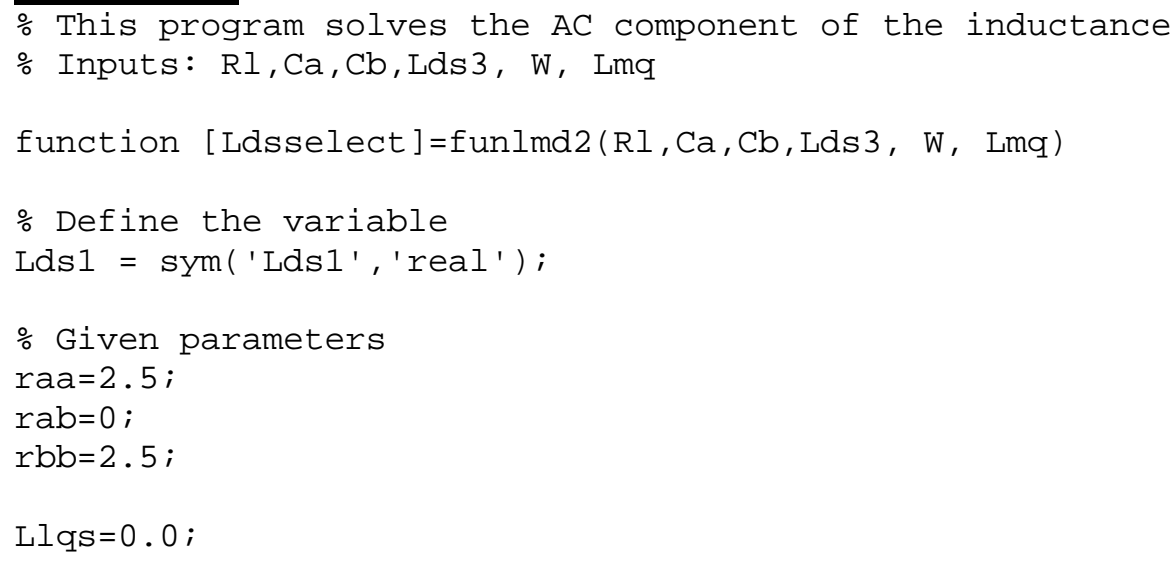




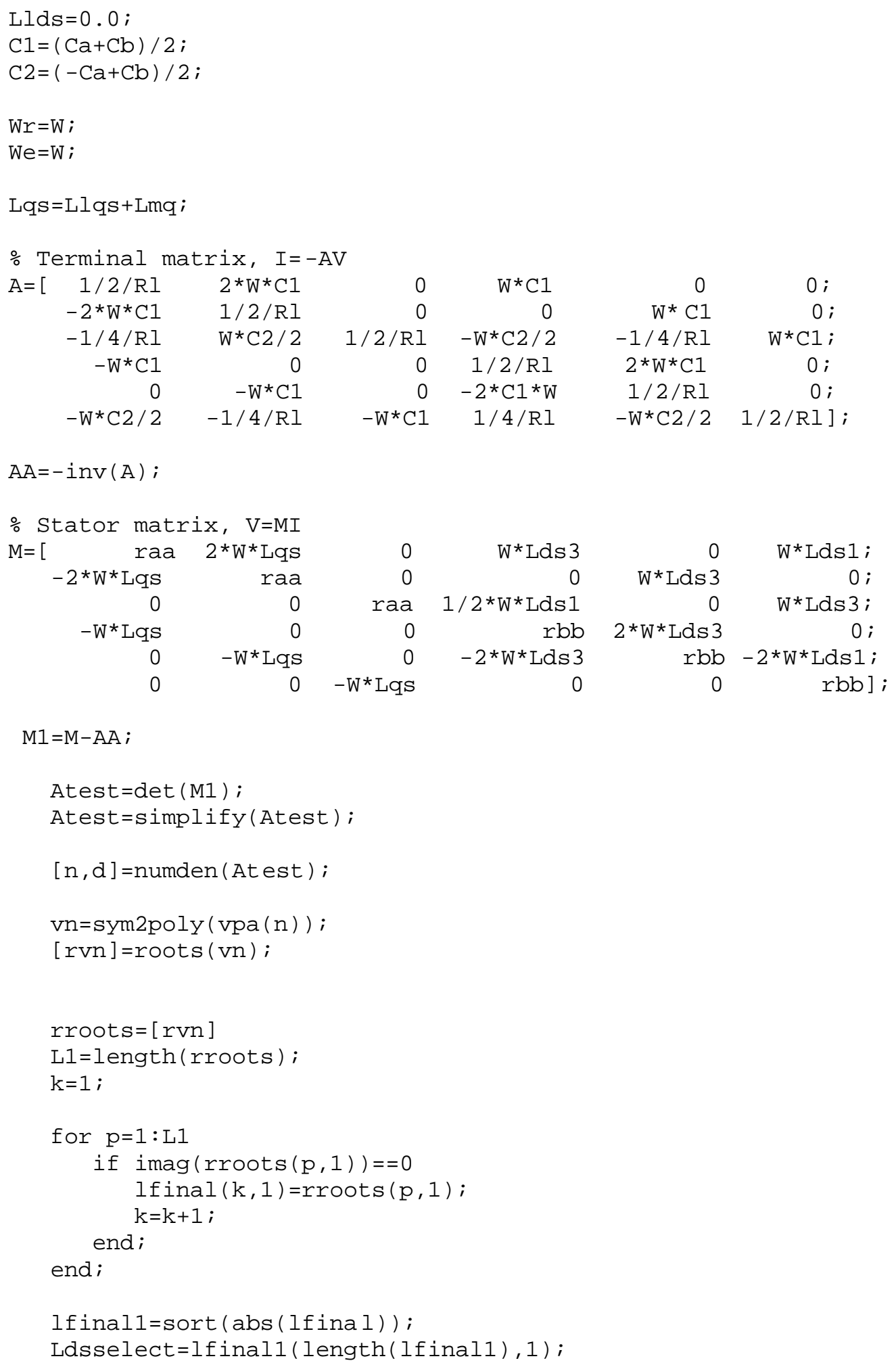

\section{steadystateRL.m}

\% This program is the main function obtaining the steady state 


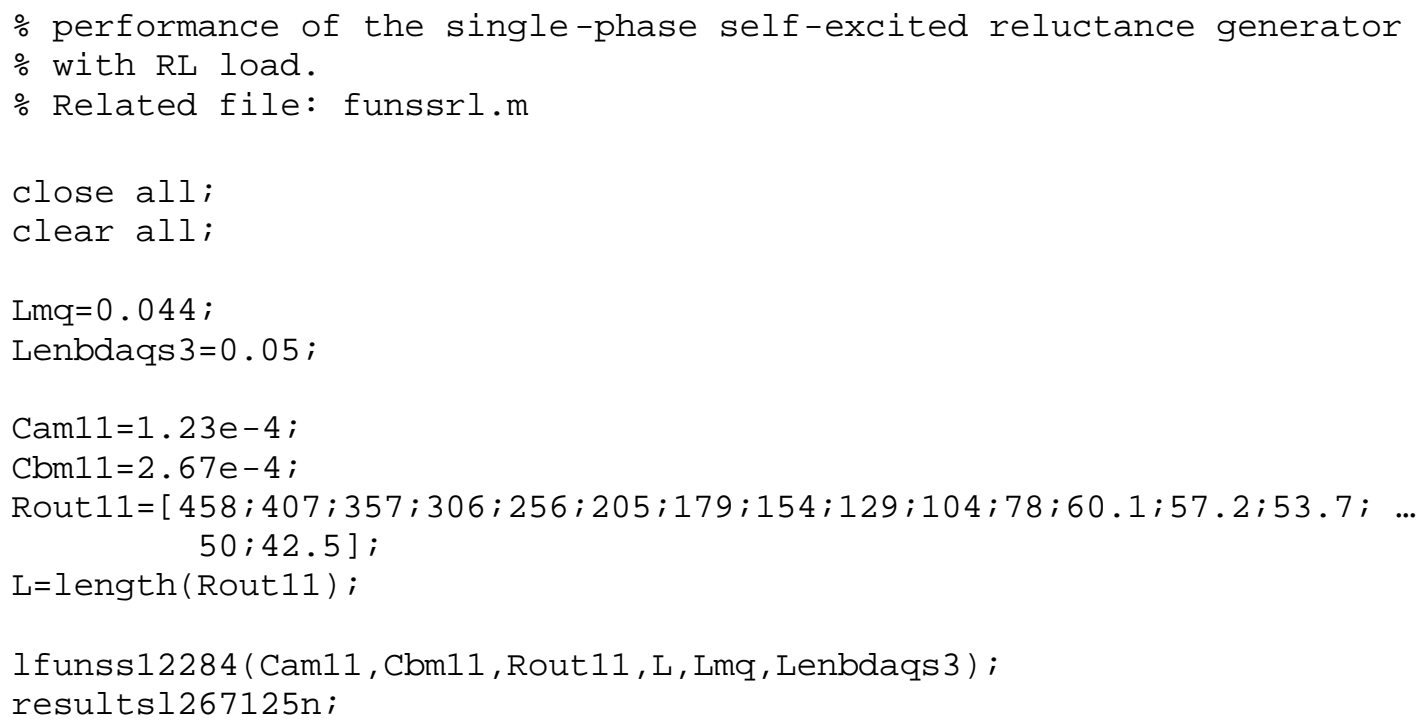

\section{funssRL.m}

\% This file has the same function as funssl.m. Because of the RL load, \% only the relevant matrixes are different. 


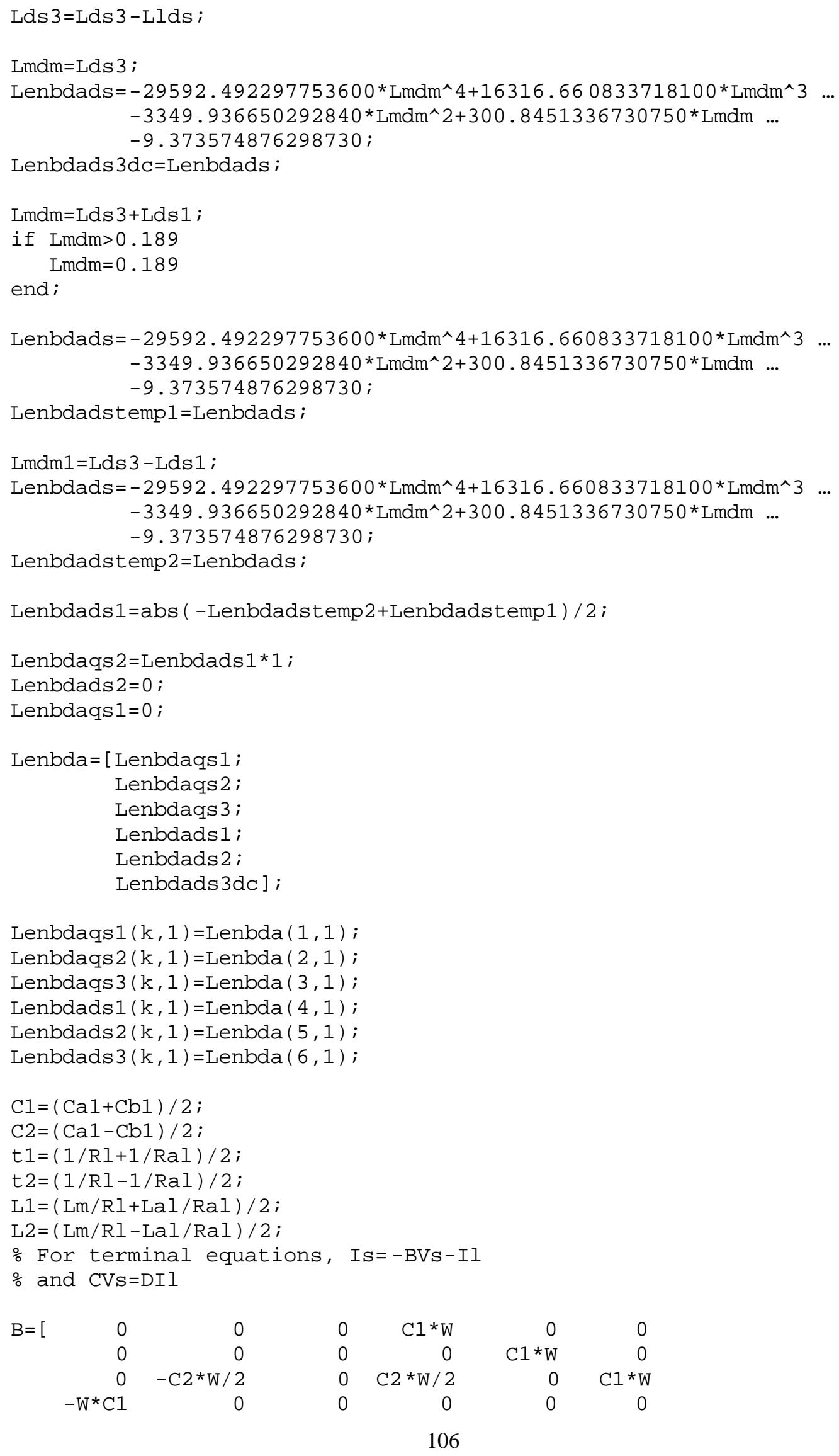




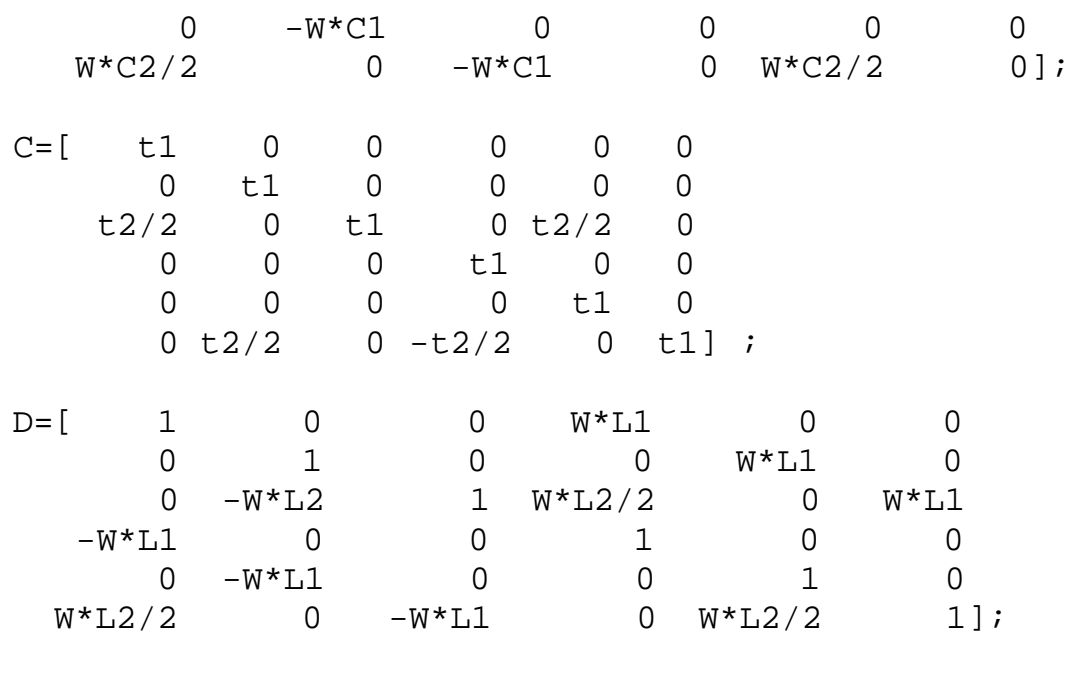

$\mathrm{AA}=-\operatorname{inv}(\mathrm{B}+\mathrm{C}$ *inv (D)) ;

ㄴenbda $=\mathrm{T}$ * Is

$\begin{array}{rrrrrc}\mathrm{T}=\text { [ Lqs+Llqs } & 0 & 0 & 0 & 0 & 0 ; \\ 0 & \text { Lqs+Llqs } & 0 & 0 & 0 & 0 ; \\ 0 & 0 & \text { Llqs+Lqs } & 0 & 0 & 0 ; \\ 0 & 0 & 0 & \text { Lds3+Llds } & 0 & \text { Lds } 1 ; \\ 0 & 0 & 0 & 0 & \text { Lds3+Llds } \\ 0 & 0 & 0 & 1 / 2 * \text { Lds } 1 & 0 & \text { Lds3+Llds]; } ; ~\end{array}$

$\mathrm{I}=\operatorname{inv}(\mathrm{T}){ }^{*}$ Lenbda;

$\operatorname{Iqs} 1(\mathrm{k}, 1)=\mathrm{I}(1,1)$;

$\operatorname{Iqs} 2(\mathrm{k}, 1)=\mathrm{I}(2,1)$;

$\operatorname{Iqs} 3(\mathrm{k}, 1)=\mathrm{I}(3,1)$;

$\operatorname{Ids} 1(\mathrm{k}, 1)=\mathrm{I}(4,1)$;

$\operatorname{Ids} 2(k, 1)=I(5,1)$;

$\operatorname{Ids} 3(k, 1)=I(6,1)$;

$\mathrm{V}=\mathrm{AA} * \mathrm{I}$;

$\operatorname{Vqs} 1(\mathrm{k}, 1)=\mathrm{V}(1,1)$;

$\operatorname{Vqs} 2(k, 1)=\operatorname{V}(2,1)$;

$\operatorname{Vqs} 3(\mathrm{k}, 1)=\mathrm{V}(3,1)$;

$\operatorname{Vds} 1(\mathrm{k}, 1)=\mathrm{V}(4,1)$;

$\operatorname{Vds} 2(\mathrm{k}, 1)=\mathrm{V}(5,1)$;

$\operatorname{Vds} 3(\mathrm{k}, 1)=\mathrm{V}(6,1)$;

$\mathrm{I} I=(\mathrm{C}$ *inv (D) ) *V;

$\operatorname{Iql} 1(k, 1)=\operatorname{Il}(1,1)$;

$\operatorname{Iql} 2(\mathrm{k}, 1)=\operatorname{Il}(2,1)$;

$\operatorname{Iql} 3(k, 1)=\operatorname{Il}(3,1)$;

$\operatorname{Idl} 1(k, 1)=\operatorname{Il}(4,1)$;

$\operatorname{Idl} 2(k, 1)=\operatorname{Il}(5,1)$;

$\operatorname{Idl} 3(k, 1)=\operatorname{Il}(6,1)$;

end;

The following loop reconstructs the waveform of the machine variables 
The same as the rest part of function funssi.m

...

$\cdots$

\section{lfunlmd1}

\% This program solves the DC component Lds3 of the inductance in $d$ axis

ㅇpots: $\mathrm{Rl}, \mathrm{Ral}, \mathrm{Ca}, \mathrm{Cb}, \mathrm{Lm}, \mathrm{Lal}, \mathrm{W}$, Lmq

function [Ldsselect]=lfunlmdl (Rl, Ral, $\mathrm{Ca}, \mathrm{Cb}, \mathrm{Lm}, \mathrm{Lal}, \mathrm{W}, \mathrm{Lmq})$

Lds $=\operatorname{sym}(' L d s '$, 'real');

$r a a=2.5$;

$\mathrm{rab}=0$;

$r \mathrm{bb}=2.5$;

$\mathrm{C} 1=(\mathrm{Ca}+\mathrm{Cb}) / 2$;

$\mathrm{C} 2=(\mathrm{Ca}-\mathrm{Cb}) / 2$;

$\mathrm{t} 1=(1 / \mathrm{Rl}+1 / \mathrm{Ra} 1) / 2$;

t2 $=(1 / \mathrm{Rl}-1 / \mathrm{Ral}) / 2$;

$\mathrm{L} 1=(\mathrm{Lm} / \mathrm{Rl}+\mathrm{Lal} / \mathrm{Ral}) / 2$;

$\mathrm{L} 2=(\mathrm{Lm} / \mathrm{Rl}-\mathrm{Lal} / \mathrm{Ral}) / 2$;

$\mathrm{Wr}=\mathrm{W}$;

$\mathrm{We}=\mathrm{W}$;

$$
\begin{aligned}
& \text { \% For terminal equations, Is }=-B V s-I l \\
& \text { and CVs=DIl }
\end{aligned}
$$

\begin{tabular}{|c|c|c|c|c|c|}
\hline$B=[$ & 0 & 0 & $\mathrm{C} 1{ }^{*} \mathrm{~W}$ & 0 & 0 \\
\hline 0 & 0 & 0 & 0 & $\mathrm{C} 1 * \mathrm{~W}$ & 0 \\
\hline 0 & $-\mathrm{C} 2 * \mathrm{~W} / 2$ & 0 & $\mathrm{C} 2 * \mathrm{~W} / 2$ & 0 & $\mathrm{C} 1 * \mathrm{~W}$ \\
\hline$-\mathrm{W} * \mathrm{C} 1$ & 0 & 0 & 0 & 0 & 0 \\
\hline 0 & $-\mathrm{W}^{\star} \mathrm{C} 1$ & 0 & 0 & 0 & 0 \\
\hline $\mathrm{W} * \mathrm{C} 2 / 2$ & 0 & $-\mathrm{W}^{\star} \mathrm{C} 1$ & 0 & $\mathrm{~W} * \mathrm{C} 2 / 2$ & $0]$ \\
\hline
\end{tabular}

\begin{tabular}{|c|c|c|c|c|c|}
\hline$D=[$ & 0 & 0 & $\mathrm{~W}^{\star} \mathrm{L} 1$ & 0 & 0 \\
\hline 0 & 1 & 0 & 0 & $W^{*} \mathrm{~L} 1$ & 0 \\
\hline 0 & $-W^{*} \mathrm{~L} 2$ & 1 & $W^{*} \mathrm{~L} 2 / 2$ & 0 & $W^{*} \mathrm{~L} 1$ \\
\hline$-W^{\star} \mathrm{L} 1$ & 0 & 0 & 1 & 0 & 0 \\
\hline 0 & $-W^{\star} \mathrm{L} 1$ & 0 & 0 & 1 & 0 \\
\hline$W^{*} \mathrm{~L} 2 / 2$ & 0 & $-W^{\star} \mathrm{L} 1$ & 0 & $W^{*} \mathrm{~L} 2 / 2$ & 1]; \\
\hline
\end{tabular}

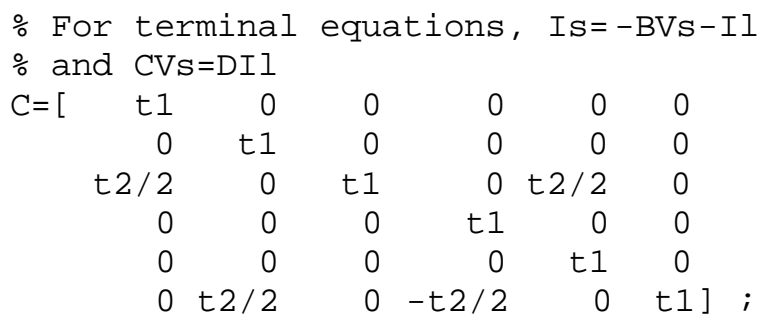

$A A=-i n v(B+C * i n v(D)) ; \circ$ the negaive sign indicates that the terminal

o currents have the opposite direction with the 


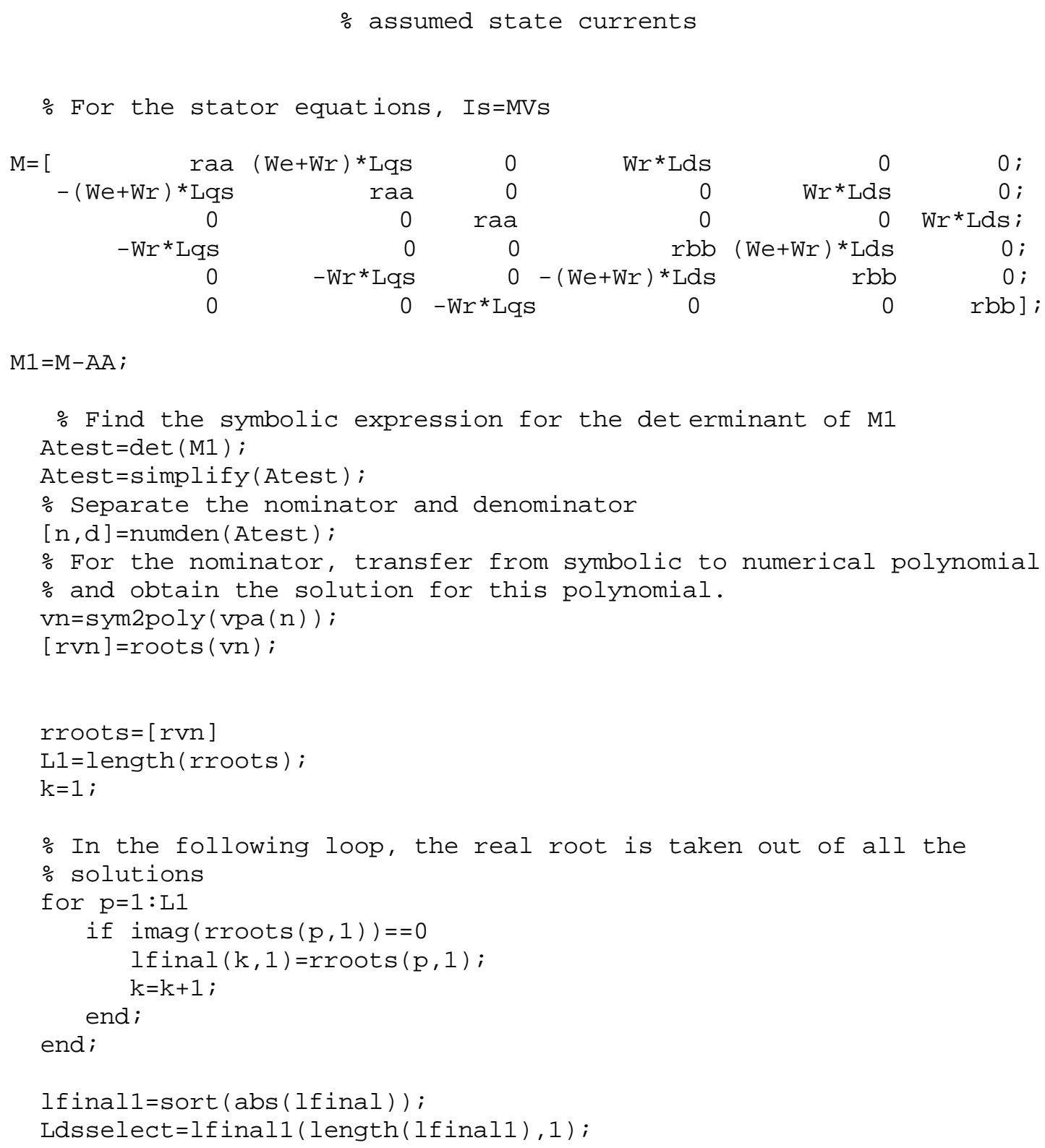

\section{lfunlmd2}

- This program solves the AC component of the inductance

Inputs: Rl, Ral, $\mathrm{Ca}, \mathrm{Cb}, \mathrm{Lm}, \mathrm{Lal}, \mathrm{Lds3}$, W, Lmq

function [Ldsselect] =lfunlmd2 ( $\mathrm{Rl}, \mathrm{Ral}, \mathrm{Ca}, \mathrm{Cb}, \mathrm{Lm}, \mathrm{Lal}, \mathrm{Lds} 3, \mathrm{~W}, \mathrm{Lmq})$

Lds1 = sym('Lds1', 'real');

raa $=2.5$;

$r a b=0$;

$r \mathrm{bb}=2.5$;

$\mathrm{C} 1=(\mathrm{Ca}+\mathrm{Cb}) / 2$;

$\mathrm{C} 2=(\mathrm{Ca}-\mathrm{Cb}) / 2$;

$t 1=(1 / \mathrm{Rl}+1 / \mathrm{Ral}) / 2$; 


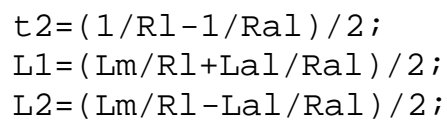




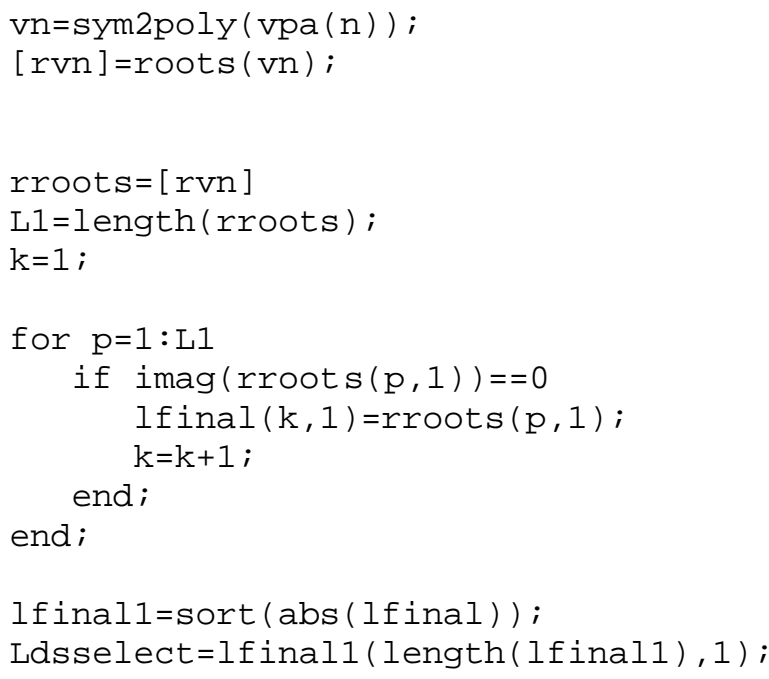

\section{Transient simulation.m:}

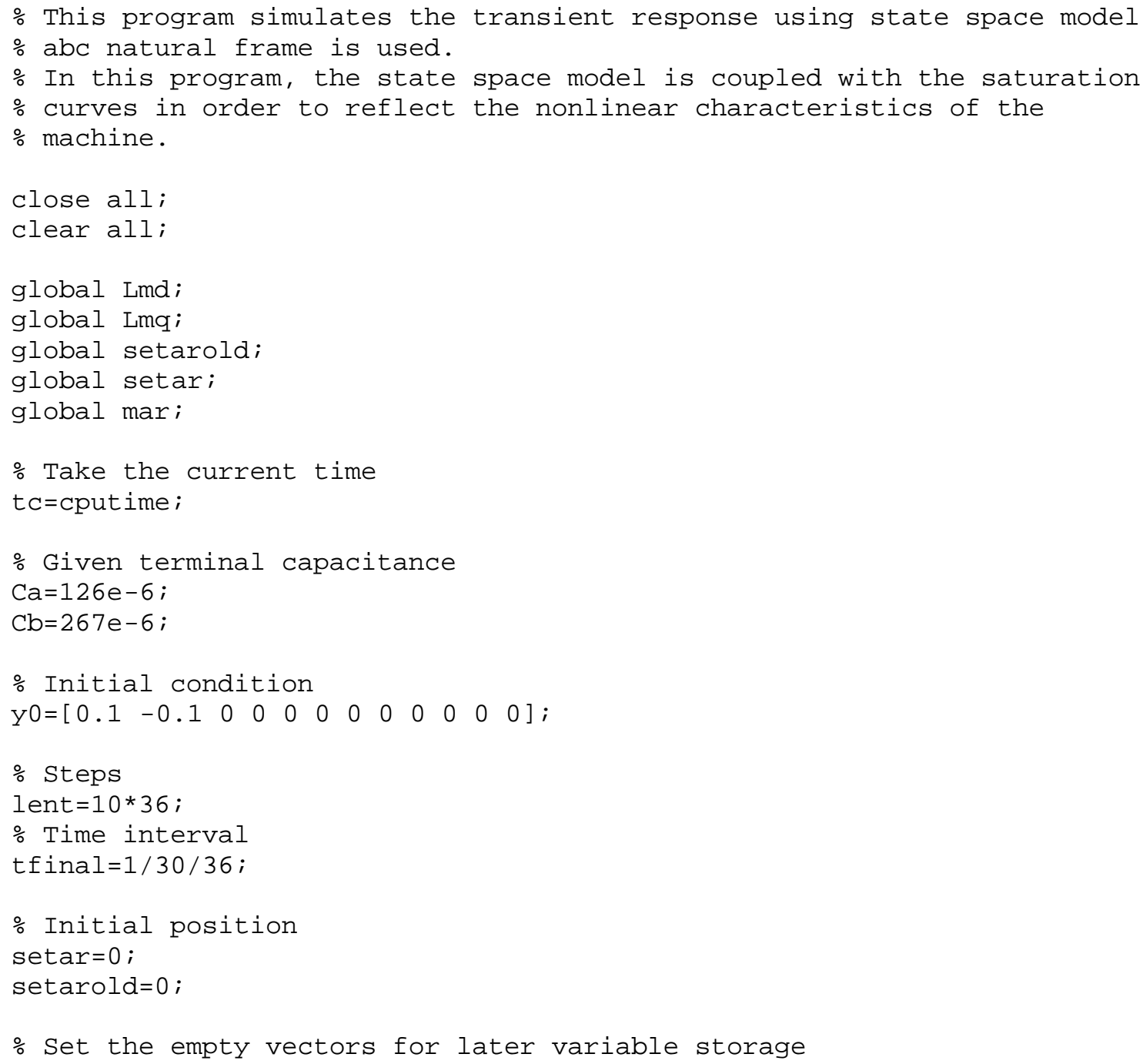




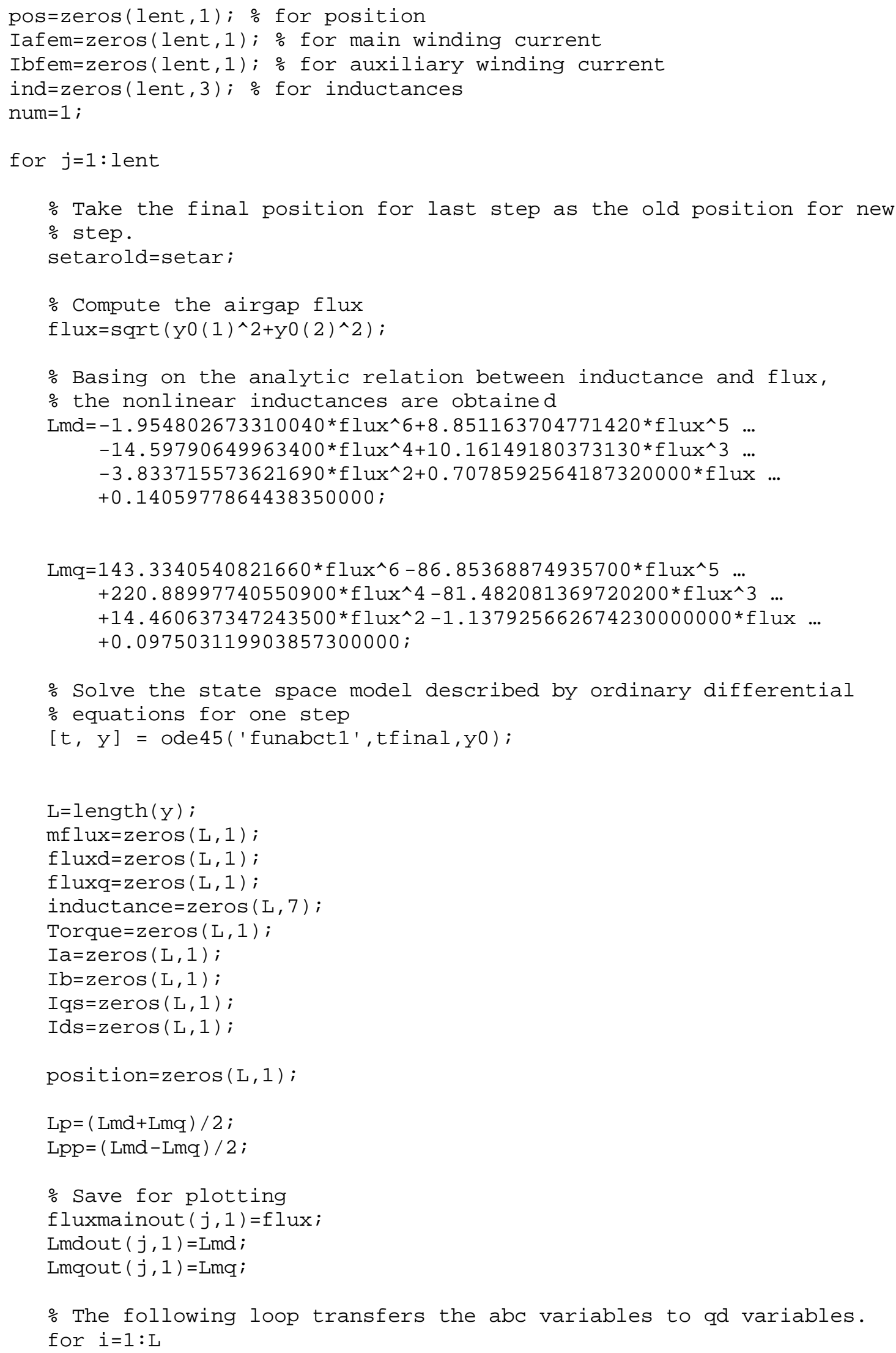




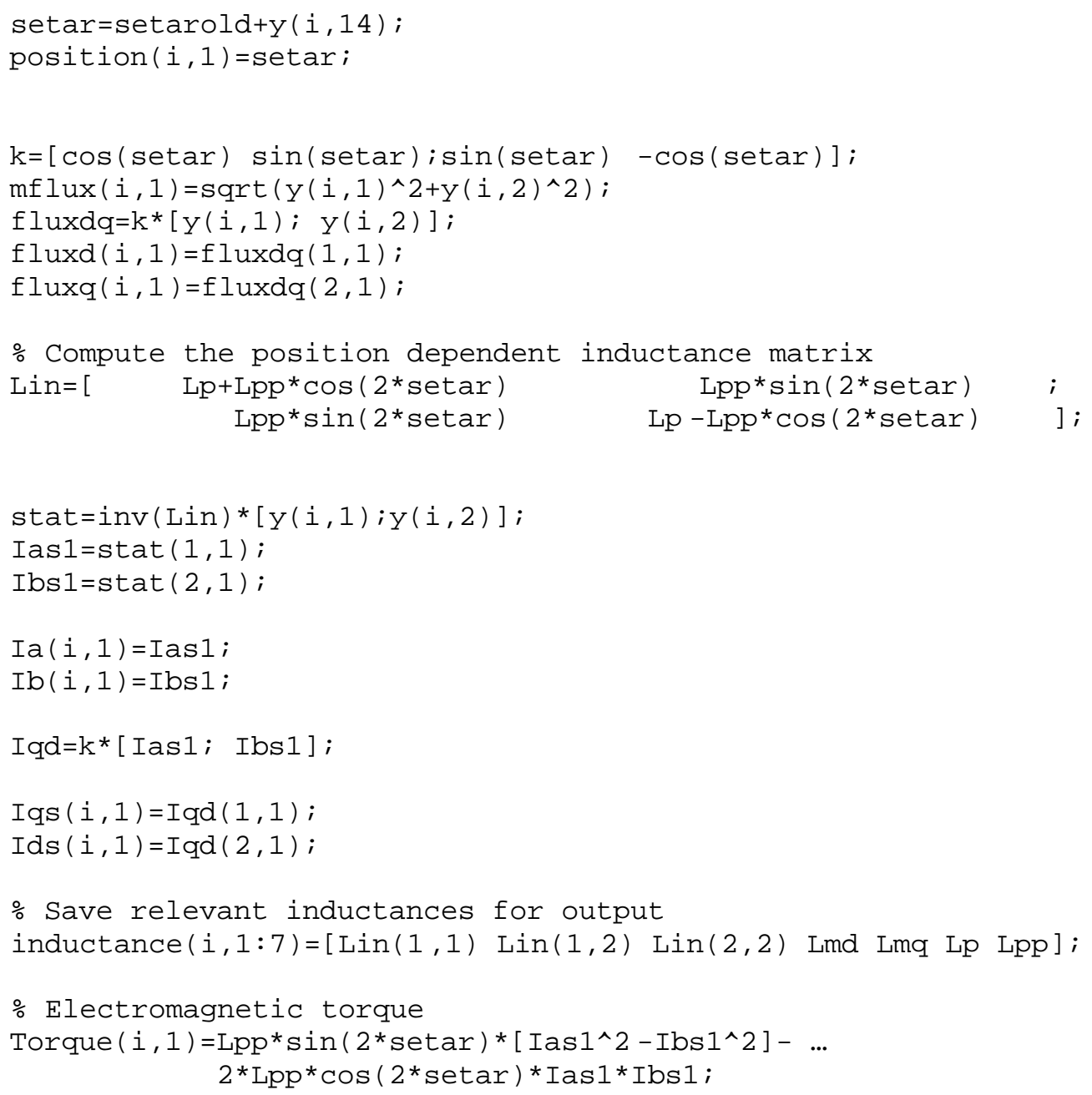




$$
\mathrm{y} 0=[\mathrm{y}(\mathrm{L}, 1: 13) 0]
$$

end;

figure (1);

plot ( $T, Y Y)$;

title('QD state for SS');

xlabel ('time');

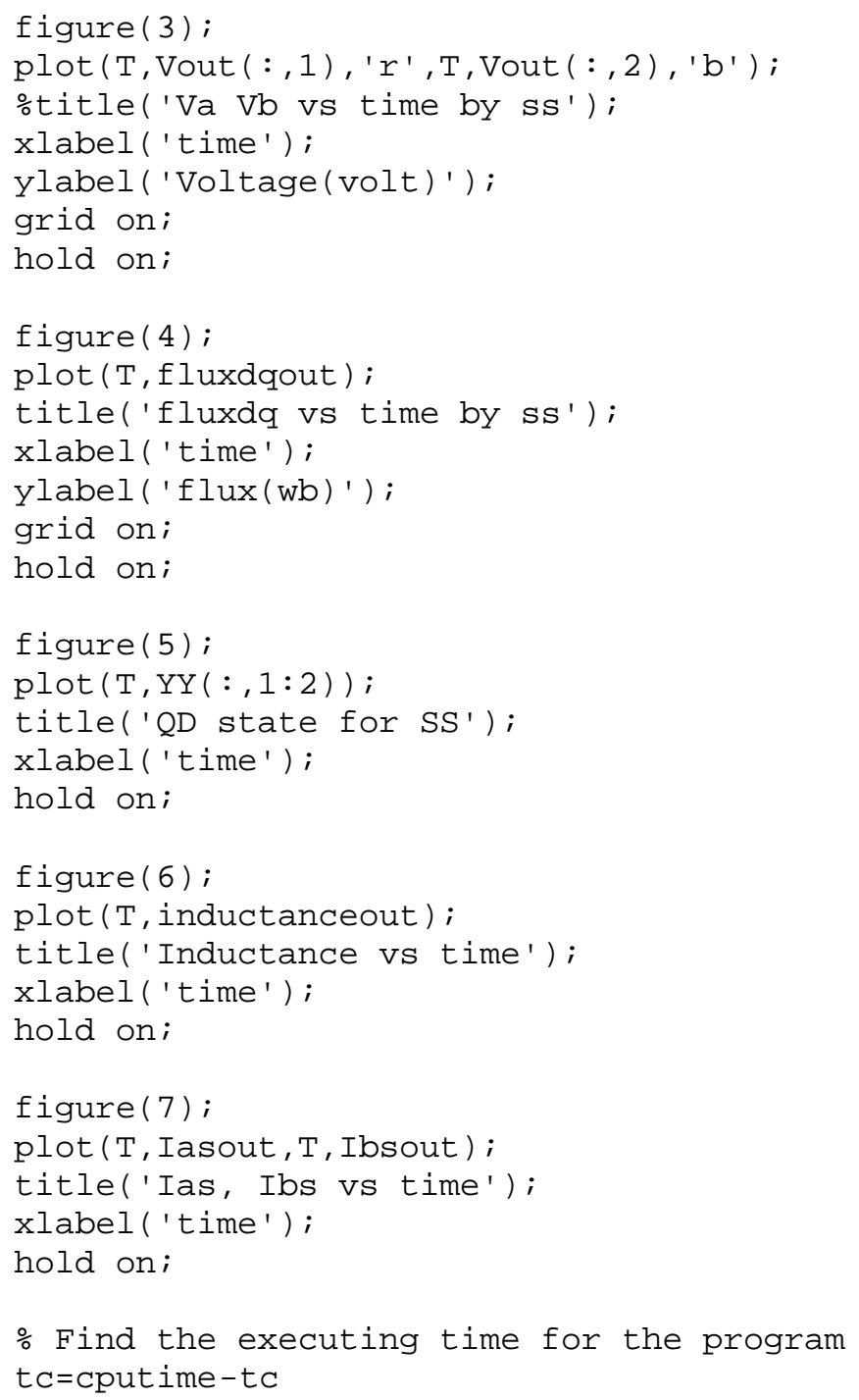

\section{funabct1.m}

function yprime $=$ funabct $1(t, y)$

global Lmd;

global Lmq;

global setarold;

global setar;

global mar; 


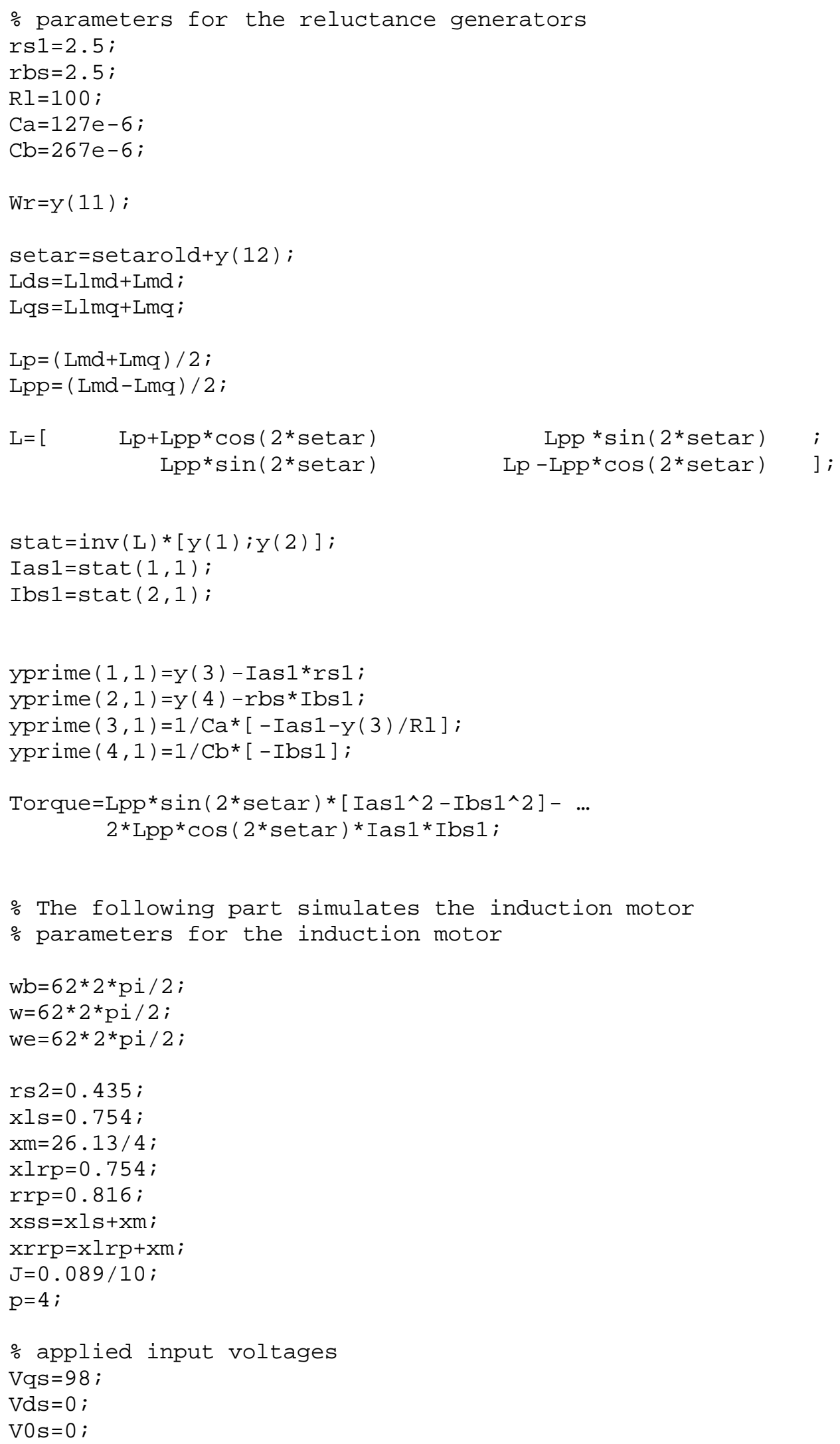




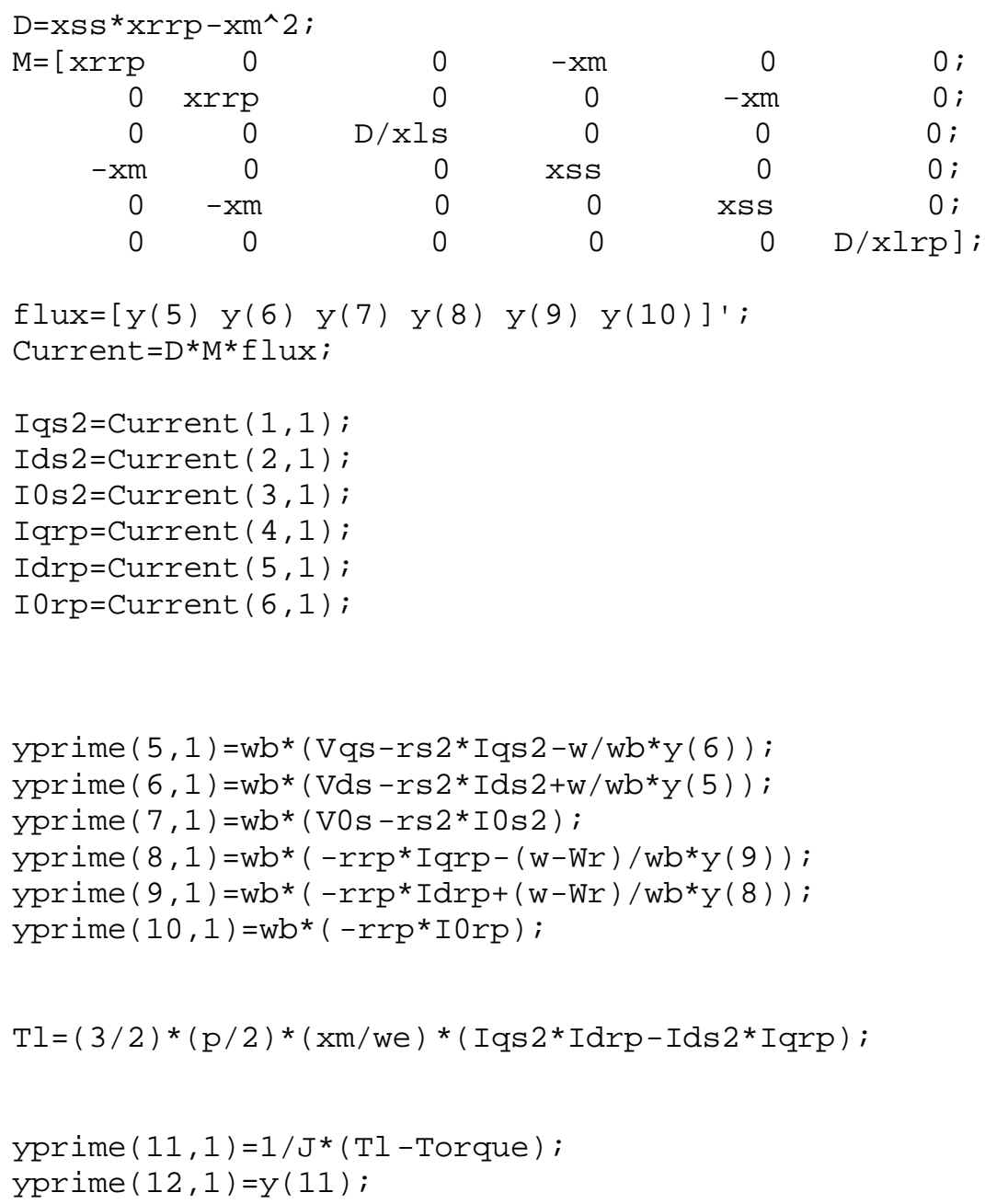

\section{FEMinductance.m}

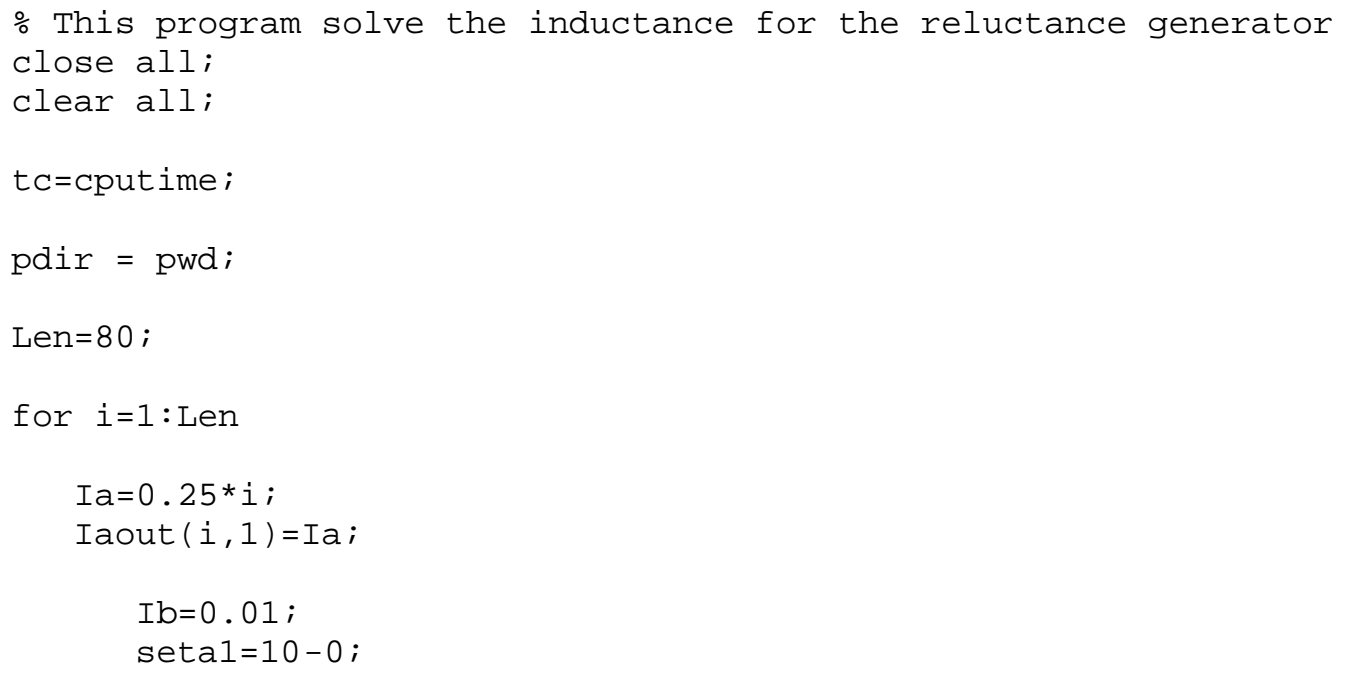




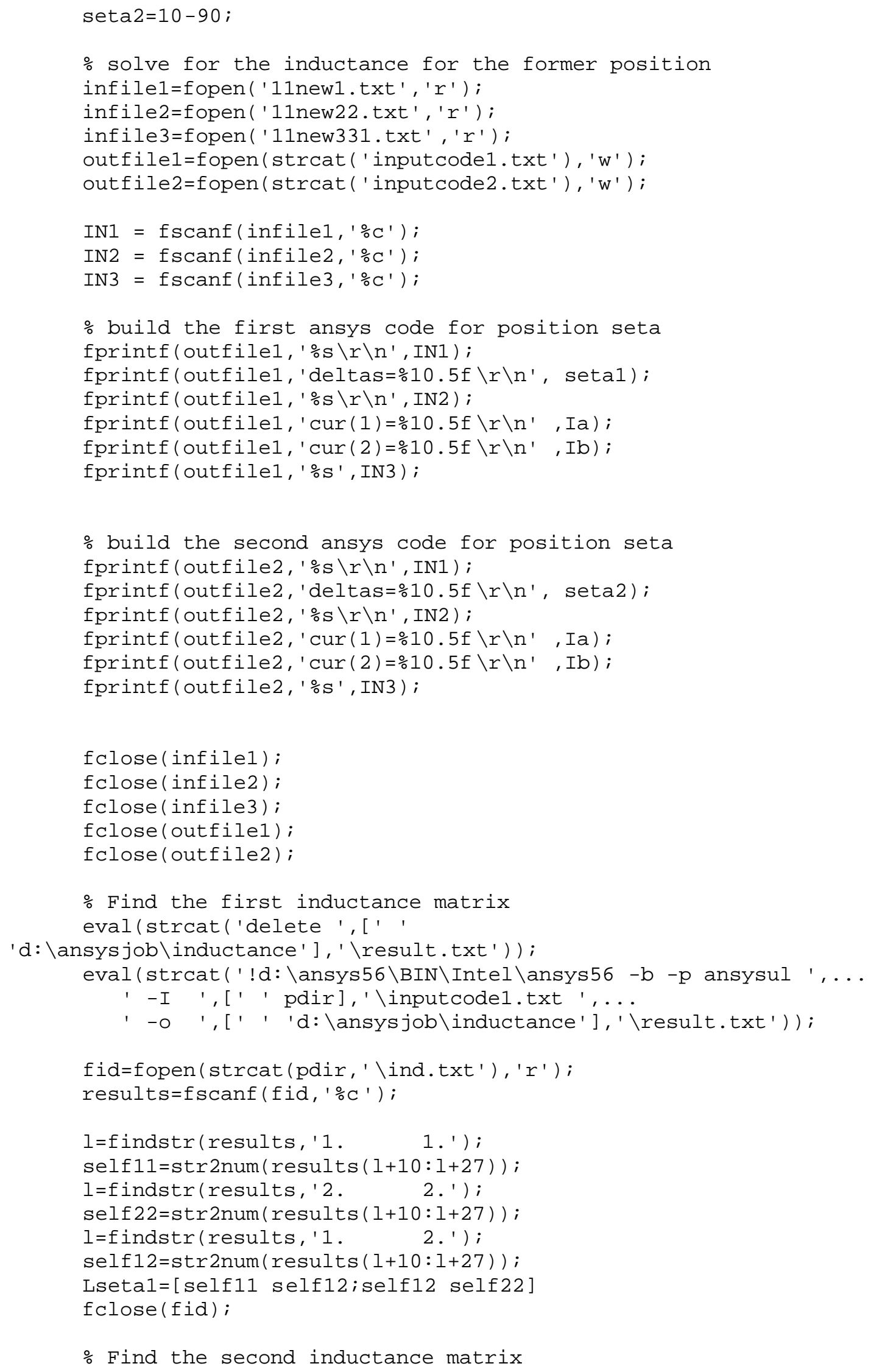




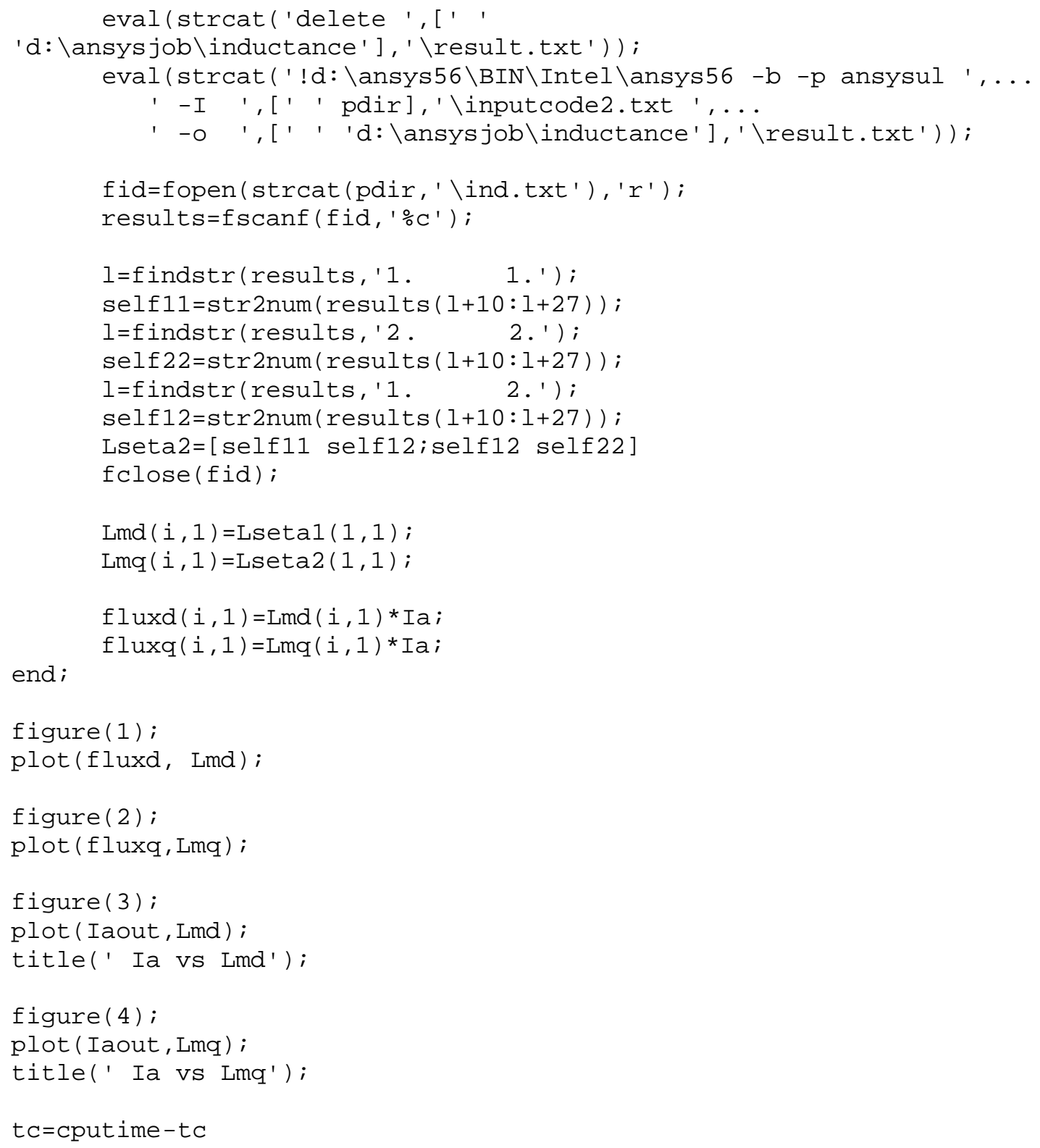

\section{1new1.txt}

/batch

lcom, $* * * * * * * * * * * * * * * * * * * * * * * * * * * * * * * * * * * * * * * * * * * * * * * * * * * * * * * * * * *$

$/ \mathrm{com}, * * * * * * * * * * * * * * * * * * * * * * * * * * * * * * * * * * * * * * * * * * * * * * * * * * * * * * * * * * *$ /com,

/com, TITLE: The Ansys program for permanent magnetic generator /com,

/com, FILENAME: 11new1.TXT

/com,

/com,

/com, 
/com, " Nonlinear Modeling and Simlation of self-excited Reluctance Generator" /com,

/com,

/com, This test explores magnetostatic analysis for PM generator

/com,

/com, Jingdong chen CSEE West Virginia University

/com,

/com, REVISION HISTORY

/com, 4/23/2000 Created

/com,

/com,

/com,

lcom, $* * * * * * * * * * * * * * * * * * * * * * * * * * * * * * * * * * * * * * * * * * * * * * * * * * * * * * * * * * *$

$/ \mathrm{com}, * * * * * * * * * * * * * * * * * * * * * * * * * * * * * * * * * * * * * * * * * * * * * * * * * * * * * * * * * * *$

/title,reluctance generator1:

$\mathrm{pi}=\operatorname{acos}(-1)$
$\mathrm{rd} 1=70.665 / 1000$
$\mathrm{rd} 2=50.465 / 1000$
$\mathrm{rd} 3=33.665 / 1000$
$\mathrm{rd} 4=33.005 / 1000$

seta $1=0$

$\operatorname{dis} 1=0$

seta2 $=3.4$

$\operatorname{dis} 2=r d 2$

seta3 $=5.1$

dis $3=r d 3$

seta $4=8.27$

dis $4=$ rd 3

seta5 $=8.27$

dis $5=$ rd 4

seta $6=-8.27$

dis $6=\mathrm{rd} 4$

seta $7=-8.27$

dis7 $=$ rd 3

seta $8=-5.1$

$\operatorname{dis} 8=\mathrm{rd} 3$

seta9 $=-3.4$

dis $9=\mathrm{rd} 2$

seta $10=3.4$

dis $10=$ rd 1

seta $11=-3.4$

dis $11=$ rd 1 


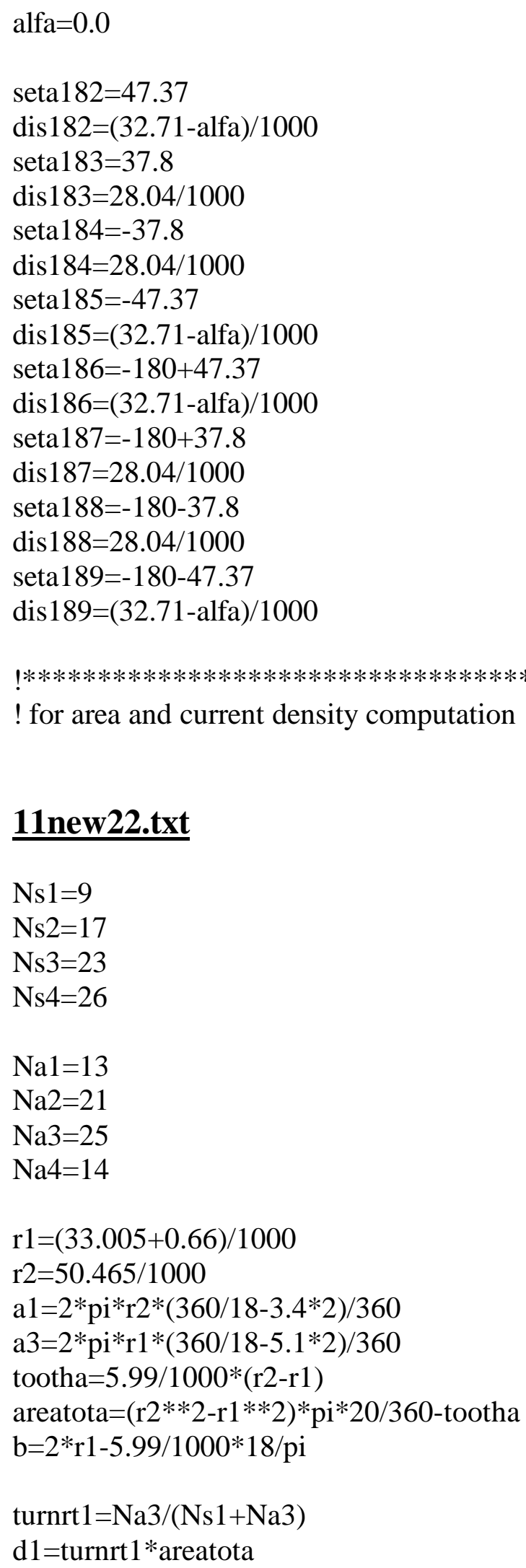

\section{$\underline{11 n e w 22 . t x t}$}

$\mathrm{Ns} 1=9$

Ns2 $=17$

$\mathrm{Ns} 3=23$

Ns4 $=26$

$\mathrm{Na} 1=13$

$\mathrm{Na} 2=21$

$\mathrm{Na} 3=25$

$\mathrm{Na} 4=14$

$\mathrm{r} 1=(33.005+0.66) / 1000$

r2 $=50.465 / 1000$

a1 $=2 *$ pi $*$ r $2 *(360 / 18-3.4 * 2) / 360$

a3 $=2 *$ pi $*$ r $1 *(360 / 18-5.1 * 2) / 360$

tootha $=5.99 / 1000 *(\mathrm{r} 2-\mathrm{r} 1)$

areatota $=(\mathrm{r} 2 * * 2-\mathrm{r} 1 * * 2) * \mathrm{pi} * 20 / 360$-tootha

$\mathrm{b}=2 * \mathrm{r} 1-5.99 / 1000 * 18 / \mathrm{pi}$

turnrt $1=\mathrm{Na} 3 /(\mathrm{Ns} 1+\mathrm{Na} 3)$

$\mathrm{d} 1=$ turnrt $1 *$ areatota 


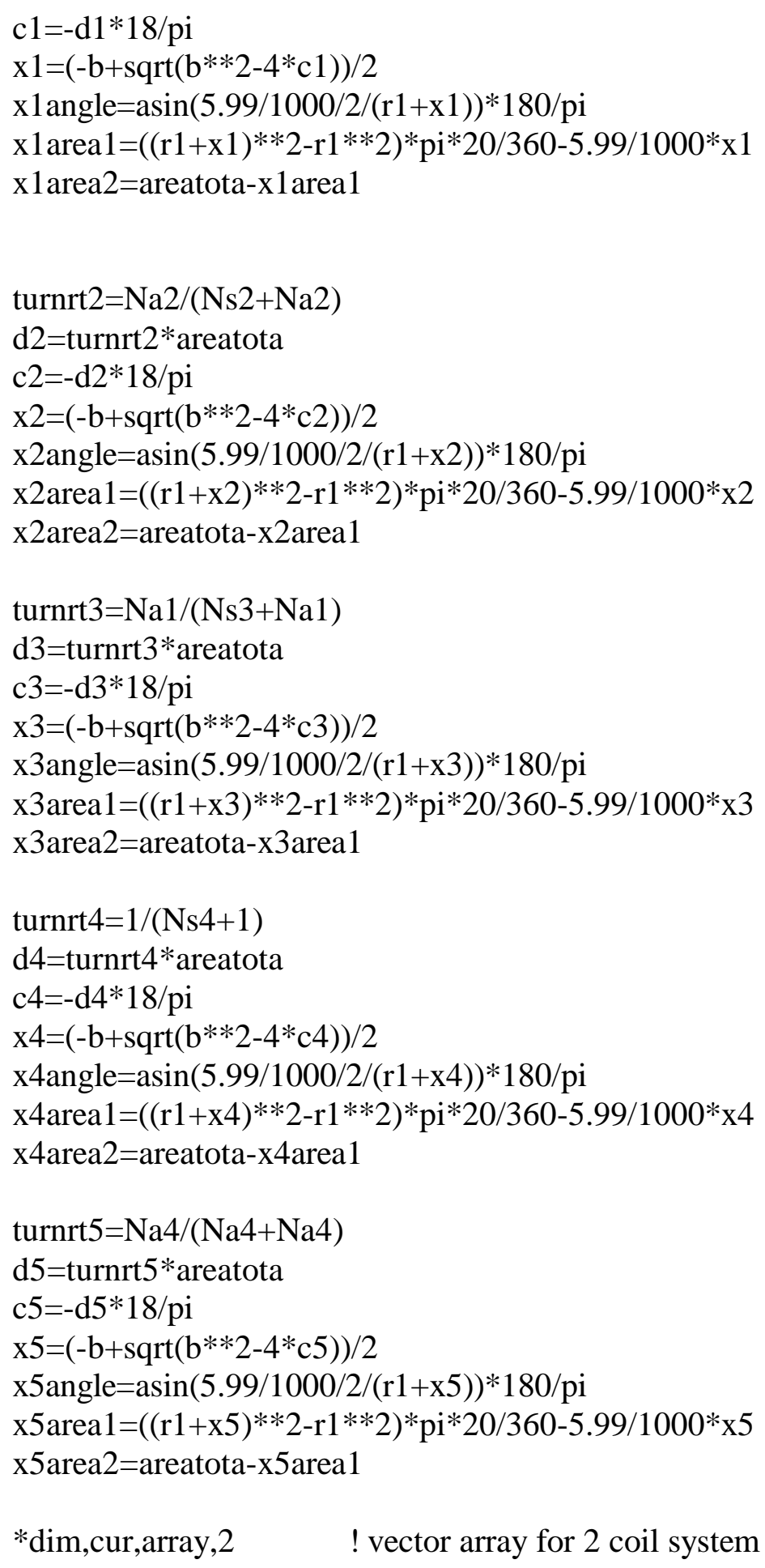

\section{$\underline{11 \text { new331.txt }}$}




$$
\begin{aligned}
& \operatorname{mjs} 1=\operatorname{cur}(1) * N s 1 / x 1 \text { area2 } \\
& \text { mjs2 }=\operatorname{cur}(1) * N s 2 / x 2 \text { area2 } \\
& \text { mjs3 }=\operatorname{cur}(1) * N s 3 / x 3 \text { area2 } \\
& \text { mjs4 }=\operatorname{cur}(1)^{* N s} 4 / x 4 \text { area2 } \\
& \text { ajs1 }=\operatorname{cur}(2) * N a 1 / x 3 \text { area1 } \\
& \text { ajs2 }=\operatorname{cur}(2) * N a 2 / x 2 \text { area1 } \\
& \text { ajs3 }=\operatorname{cur}(2) * N a 3 / x 1 \text { area1 } \\
& \text { ajs4 }=\operatorname{cur}(2) * N a 4 / x 5 \text { area1 }
\end{aligned}
$$

symfac $=0.110$

factor

/prep7

csys, 1

!Set to cart coordinates:

cylindal

/com, ------------- Set Element Type ----

et,1,plane53,0 ! Define element Type

! Reference Number

$: 1$

: plane13

! Element Name

$\begin{array}{lll} & ! 0- & \text { AZ Degree } \\ \text { of Freedom } & ! 0- & \text { Include }\end{array}$

extra shapes

/com,-----------define material properties-

emunit,mks !emag unit,

default free space mu

mp,murx, 1,2000

!mp,murx,2,1000

steel, relative mu mat2

mp,murx, 3, 1

relative $\mathrm{mu} \quad$ mat3

mp,murx, $4,1 \quad$ !air, relative

$\mathrm{mu} \quad$ mat4

/com,---- efine the B-H curve for $24 \mathrm{~m} 19$

tb,bh,2

$$
\begin{aligned}
& \text { tbpt, }, 46.1564,0.4 \\
& \text { tbpt, }, 52.5228,0.5 \\
& \text { tbpt, }, 59.685,0.6 \\
& \text { tbpt,,66.0514,0.7 } \\
& \text { tbpt,,72.4178,0.8 } \\
& \text { tbpt,,79.58,0.9 } \\
& \text { tbpt,,87.538, } \\
& \text { tbpt,,97.0876,1.1 } \\
& \text { tbpt,,107.433,1.2 } \\
& \text { tbpt,,121.7574,1.3 } \\
& \text { tbpt,,159.16,1.4 } \\
& \text { tbpt,,270.572,1.5 } \\
& \text { tbpt,,648.577,1.6 } \\
& \text { tbpt,,2000.6412,1.7 } \\
& \text { tbpt,,4440.564,1.8 }
\end{aligned}
$$

/com,---- Generate Keypoints ----

csys, 1

$\mathrm{k}, 1$, dis1, seta1, 0

k, 2, dis $2, \operatorname{seta} 2,0$

$\mathrm{k}, 3$, dis3, seta3, 0

$\mathrm{k}, 4$, dis4, seta4, 0

$\mathrm{k}, 5$, dis 5, seta5, 0

$\mathrm{k}, 6$, dis6, seta6, 0

$\mathrm{k}, 7$, dis7, seta7, 0

$\mathrm{k}, 8$, dis8, seta8, 0

$\mathrm{k}, 9$, dis9, seta9, 0

$\mathrm{k}, 10$, dis 10, seta10, 0

$\mathrm{k}, 11$, dis 11 , seta11, 0

k, 182,dis182,seta182+deltas, 0

k,183,dis183,seta183+deltas,0

k, 184,dis184,seta184+deltas,0

k, 185,dis185,seta185+deltas, 0

k,186,dis186,seta186+deltas,0

k, 187,dis187,seta187+deltas,0

$\mathrm{k}, 188$,dis188,seta188+deltas, 0

!copper, $\quad$ k,189,dis189, seta189+deltas,0

$$
\begin{aligned}
& \mathrm{k}, 230,19.14 / 2 / 1000,0,0 \\
& \mathrm{k}, 231,19.14 / 2 / 1000,90,0 \\
& \mathrm{k}, 232,19.14 / 2 / 1000,180,0 \\
& \mathrm{k}, 233,19.14 / 2 / 1000,270,0
\end{aligned}
$$




\begin{tabular}{|c|c|}
\hline k,191,r1+x5,x5angle,0 & $\mathrm{a}, 3+$ counta, $4+$ counta, $5+$ counta, $6+$ counta \\
\hline $\mathrm{k}, 192, \mathrm{r} 1+\mathrm{x} 5,20-\mathrm{x} 5$ angle, 0 &, $7+$ counta, $8+$ counta \\
\hline $\mathrm{k}, 193, \mathrm{r} 1+\mathrm{x} 1,20+\mathrm{x} 1$ angle, 0 & *enddo \\
\hline k,194,r1+x1,40-x1 angle,0 & \\
\hline $\mathrm{k}, 195, \mathrm{r} 1+\mathrm{x} 2,40+\mathrm{x} 2$ angle, 0 & $*$ do, countb, $0,160,10$ \\
\hline $\mathrm{k}, 196, \mathrm{r} 1+\mathrm{x} 2,60-\mathrm{x} 2$ angle, 0 & $\mathrm{a}, 2+$ countb, $191+$ countb/5,192+countb/5, \\
\hline $\mathrm{k}, 197, \mathrm{r} 1+\mathrm{x} 3,60+\mathrm{x} 3$ angle, 0 & $19+$ countb \\
\hline $\mathrm{k}, 198, \mathrm{r} 1+\mathrm{x} 3,80-\mathrm{x} 3$ angle, 0 & $\mathrm{a}, 191+$ countb/5,3+countb, $4+$ countb, $5+\mathrm{c}$ \\
\hline $\mathrm{k}, 199, \mathrm{r} 1+\mathrm{x} 4,80+\mathrm{x} 4$ angle, 0 & ountb, $16+$ countb, $17+$ countb, $18+$ countb \\
\hline $\mathrm{k}, 200, \mathrm{r} 1+\mathrm{x} 4,100-\mathrm{x} 4$ angle, 0 & $192+$ countb $/ 5$ \\
\hline $\mathrm{k}, 201, \mathrm{r} 1+\mathrm{x} 4,100+\mathrm{x} 4$ angle, 0 & *enddo \\
\hline k,202,r1+x4,120-x4angle,0 & \\
\hline $\mathrm{k}, 203, \mathrm{r} 1+\mathrm{x} 3,120+\mathrm{x} 3$ angle, 0 & $a, 172,225,190,9$ \\
\hline $\mathrm{k}, 204, \mathrm{r} 1+\mathrm{x} 3,140-\mathrm{x} 3$ angle, 0 & a, $225,173,174,175,6,7,8,190$ \\
\hline $\mathrm{k}, 205, \mathrm{r} 1+\mathrm{x} 2,140+\mathrm{x} 2$ angle, 0 & \\
\hline $\mathrm{k}, 206, \mathrm{r} 1+\mathrm{x} 2,160-\mathrm{x} 2$ angle, 0 & \\
\hline $\mathrm{k}, 207, \mathrm{r} 1+\mathrm{x} 1,160+\mathrm{x} 1$ angle, 0 & $a, 2,12,22,32,42,52,62,72,82,92,100,90,8$ \\
\hline $\mathrm{k}, 208, \mathrm{r} 1+\mathrm{x} 1,180-\mathrm{x} 1$ angle, 0 & $0,70,60,50,20,10$ \\
\hline $\mathrm{k}, 209, \mathrm{r} 1+\mathrm{x} 5,180+\mathrm{x} 5$ angle, 0 & $\mathrm{a}, 2,172,162,142,132,122,112,102,92,100$ \\
\hline k,210,r1+x5,200-x5angle,0 & $, 110,120,130,150,160,170,180,10$ \\
\hline $\mathrm{k}, 211, \mathrm{r} 1+\mathrm{x} 1,200+\mathrm{x} 1$ angle, 0 & ! rotor \\
\hline $\mathrm{k}, 212, \mathrm{r} 1+\mathrm{x} 1,220-\mathrm{x} 1$ angle, 0 & \\
\hline $\mathrm{k}, 213, \mathrm{r} 1+\mathrm{x} 2,220+\mathrm{x} 2$ angle, 0 & $\mathrm{a}, 182,183,184,185$ \\
\hline $\mathrm{k}, 214, \mathrm{r} 1+\mathrm{x} 2,240-\mathrm{x} 2$ angle, 0 & $\mathrm{a}, 186,187,188,189$ \\
\hline $\mathrm{k}, 215, \mathrm{r} 1+\mathrm{x} 3,240+\mathrm{x} 3$ angle, 0 & \\
\hline $\mathrm{k}, 216, \mathrm{r} 1+\mathrm{x} 3,260-\mathrm{x} 3$ angle, 0 & csys, 0 \\
\hline $\mathrm{k}, 217, \mathrm{r} 1+\mathrm{x} 4,260+\mathrm{x} 4$ angle, 0 & $\mathrm{a}, 183,184,187,188$ \\
\hline $\mathrm{k}, 218, \mathrm{r} 1+\mathrm{x} 4,280-\mathrm{x} 4$ angle, 0 & csys, 1 \\
\hline $\mathrm{k}, 219, \mathrm{r} 1+\mathrm{x} 4,280+\mathrm{x} 4$ angle, 0 & \\
\hline $\mathrm{k}, 220, \mathrm{r} 1+\mathrm{x} 4,300-\mathrm{x} 4$ angle, 0 & $\mathrm{a}, 182,185,186,189$ \\
\hline $\mathrm{k}, 221, \mathrm{r} 1+\mathrm{x} 3,300+\mathrm{x} 3$ angle, 0 & $a, 6,5,26,25,46,45,66,65,86,85,106,105,1$ \\
\hline k,222,r1+x3,320-x3angle,0 & $26,125,146,145,166,165$ \\
\hline $\mathrm{k}, 223, \mathrm{r} 1+\mathrm{x} 2,320+\mathrm{x} 2$ angle, 0 & $\mathrm{a}, 230,231,232,233$ \\
\hline $\mathrm{k}, 224, \mathrm{r} 1+\mathrm{x} 2,340-\mathrm{x} 2$ angle, 0 & \\
\hline $\mathrm{k}, 225, \mathrm{r} 1+\mathrm{x} 1,340+\mathrm{x} 1$ angle, 0 & allsel \\
\hline k,190,r1+x1,360-x1 angle,0 & aovlap,all \\
\hline & numcmp,area \\
\hline & /pnum,area, 1 \\
\hline$*$ do, count, $-20,-340,-20$ & aplot \\
\hline local, $11,1,0,0,0$, count $, 0,0$ & \\
\hline ktran, $1,2,11,1,0,0,0$ & /com,-----assign meterial to areas \\
\hline *enddo & /com,---- Create Named Areas ---- \\
\hline$*$ do, counta, $0,170,10$ & \\
\hline a, $2+$ counta, $191+$ counta $/ 5,3+$ counta, $8+$ co & $*$ do, count, $1,36,1$ \\
\hline
\end{tabular}




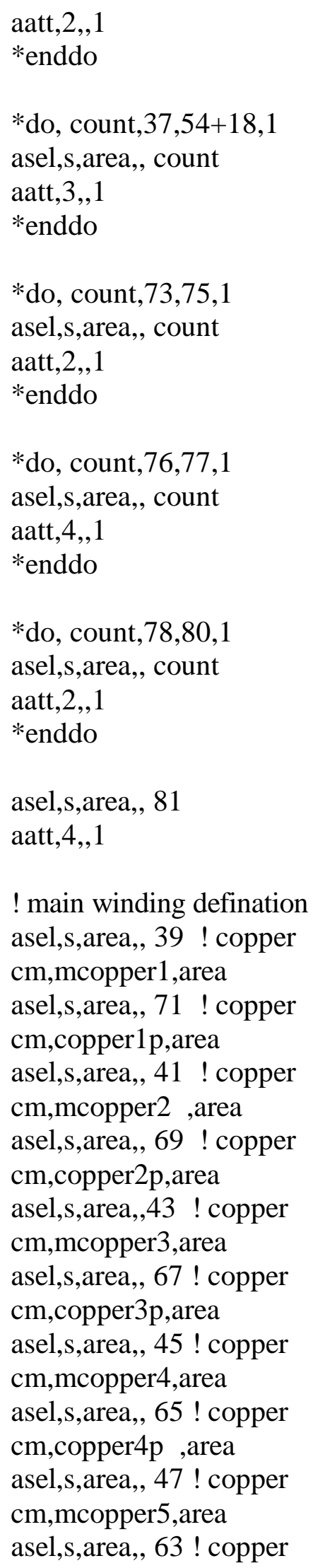

$$
\begin{aligned}
& \text { cm,copper5p, area } \\
& \text { asel,s,area,, } 49 \text { ! copper } \\
& \mathrm{cm} \text {,mcopper6, area } \\
& \text { asel,s,area,, } 61 \text { ! copper } \\
& \mathrm{cm} \text {,copplstolectreaoth } \\
& \text { asel,s,area,, } 51 \text { ! copper } \\
& \text { cm,mcopper7, area } \\
& \text { asel,s,area,, } 59 \text { ! copper } \\
& \mathrm{cm}, \text { copper } 7 \mathrm{p} \text {, area } \\
& \text { asel,s,aresejløot tocopper } \\
& \text { cm,mcopper8, area } \\
& \text { asel,s,area,, } 57 \text { ! copper } \\
& \mathrm{cm} \text {,copper8p, area }
\end{aligned}
$$

! Start wisellect defithation asel,s,area,, 44 ! copper $\mathrm{cm}$,acopper1, area asel,s,area,, 50 ! copper cm,copper1d, area asel,s,arkSelldat tocotpper $\mathrm{cm}$, acopper2, area asel,s,area,, 52 ! copper $\mathrm{cm}$, copper2d,area asel,s,arkSellad tocopper $\mathrm{cm}$,acopper3, area asel,s,area,, 54 ! copper cm, copper $3 \mathrm{~d}$, area asel,s,area,, 38 ! copper $\mathrm{cm}$, acopper4, area asel,s,area,, 56 ! copper cm,copper4d,area asel,s,area,, 37 ! copper cm, acopper5, area asel,s,area,, 55 ! copper $\mathrm{cm}$,copper5d,area asel,s,area,, 72 ! copper $\mathrm{cm}$, acopper6, area asel,s,area,, 58 ! copper $\mathrm{cm}$,copper6d,area asel,s,area,, 70 ! copper cm,acopper7 , area asel,s,area,, 60 ! copper cm,copper7d, area asel,s,area,, 68 ! copper $\mathrm{cm}$, acopper8, area asel,s,area,, 62 ! copper 


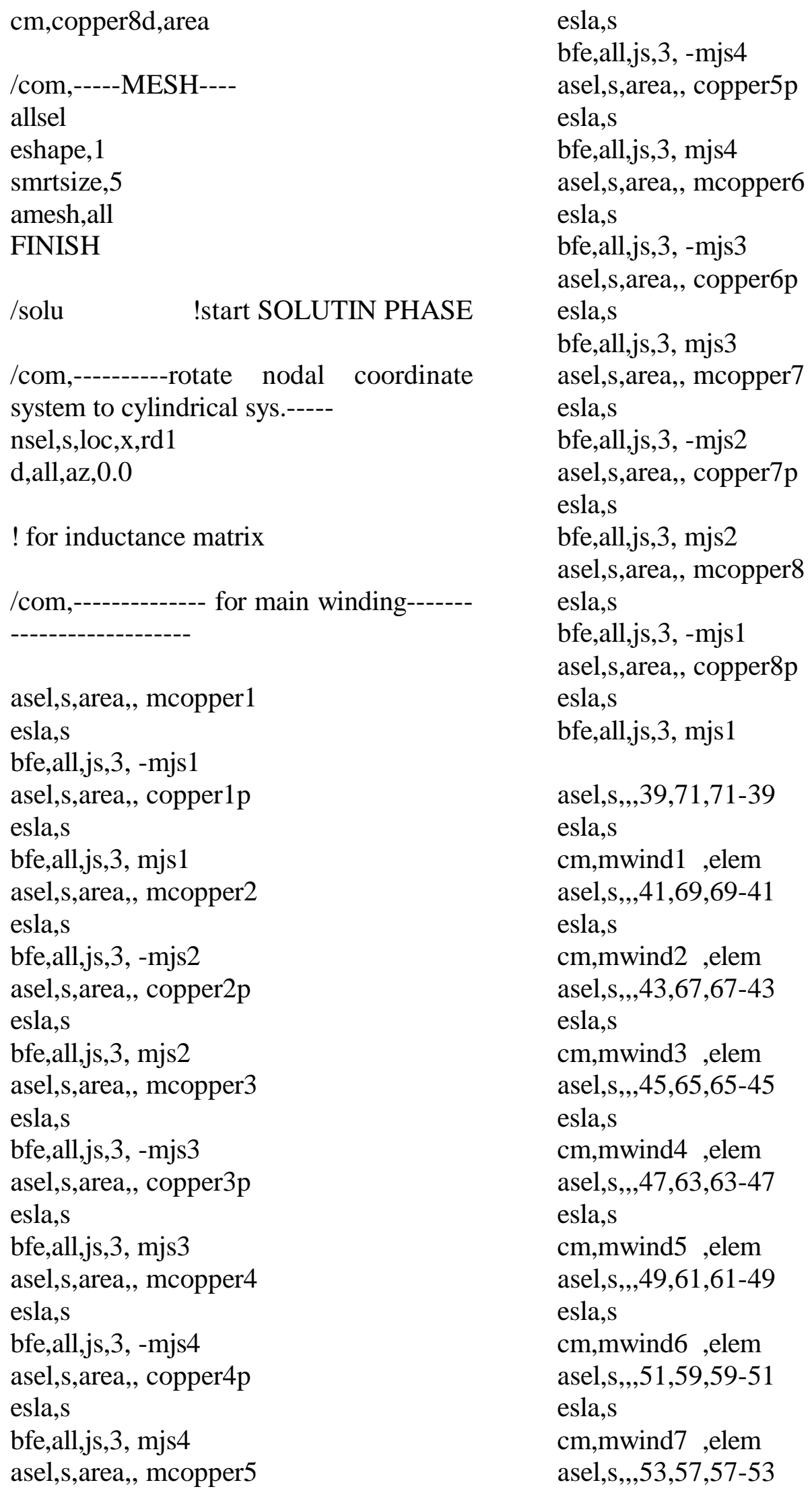


esla,s
cm,mwind8 ,elem
cmgrp,winding1,mwind1,mwind2,mwin
d3,mwind4,mwind5,mwind6,mwind7,m
wind8
!cmgrp,winding2,mwind5,mwind6,mwin
d7,mwind8

/com,--------------- for auxiliary winding--

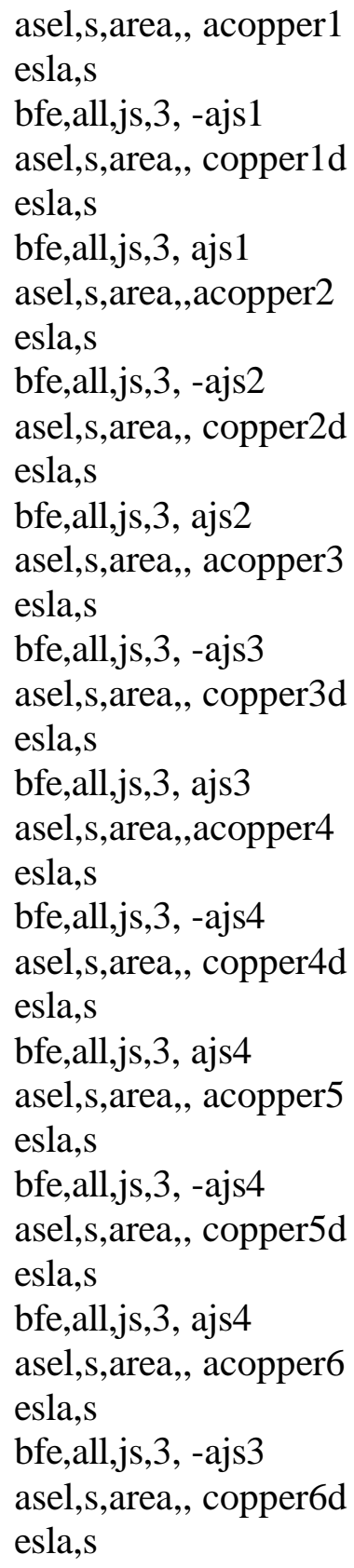

bfe,all,js,3, ajs3

asel,s,area,, acopper7

esla,s

bfe,all,js,3, -ajs2

asel,s, area, copper7d

esla,s

bfe,all,js,3, ajs2

asel,s,area,, acopper8

esla,s

bfe,all,js,3, -ajs1

asel,s,area, copper8d

esla,s

bfe,all,js,3, ajs1

! auxiliary winding

asel,s,,,44,50,50-44

esla,s

cm,awind1 ,elem

asel,s,,,42,52,52-42

esla,s

cm,awind2, elem

asel,s,,,40,54,54-40

esla,s

cm,awind3 ,elem

asel,s,,,38,56,56-38

esla,s

cm,awind4, elem

asel,s, ,,37,55,55-37

esla,s

cm,awind5, elem

asel,s,,,58,72,72-58

esla,s

cm,awind6, elem

asel,s,,,60,70,70-60

esla,s

cm,awind7 ,elem

asel,s,,,62,68,68-62

esla,s

cm,awind8, elem

cmgrp, winding2, awind1, awind2, awind3, awind4, awind5, awind6, awind7, awind8 !cmgrp,winding4, awind5, awind6, awind7 ,awind8

allsel 
MAGSOLV,0,3,0.001, ,25,

\section{FINISH}

/POST1

rsys, 1

PLF2D, 27, 0,10,1

finish

lmatrix,symfac,'winding','cur','ind' ! compute inductance matrix

*stat,ind ! list entries of

inductance matrix, ind 


\section{Jingdong Chen}

381 Newton Avenue, Apartment 104

Morgantown, West Virginia 26505

ïngdong@ieee.org, jingdongc@hotmail.com

Day (304) 293-6371x2546, Evening (304) 293-5147

Homepage: http//www.csee.wvu.edu/ jingdong

\section{OBJECTIVE:}

Seeking a post doctorate position in electrical engineering, especially interested in the following fields: electric machine analysis and design, electric machine control and drive, hybrid electric vehicle, power electronics, power system, MEMS control.

\section{EDUCATION:}

Ph.D., Feb. 2001 (expected) WEST VIRGINIA UNIVERSITY

M. S., Mar. 1993

B. S., July. 1990 HARBIN INSTITUTE of TECHNOLOGY

GPA: $4.0 / 4.0$

GPA: $3.7 / 4.0$

GPA: $3.7 / 4.0$

Dissertation Title: "Nonlinear Transient and Steady State Analysis for Self-excited Single-phase Synchronous Reluctance Generator ",

Advisor: Dr. Parviz Famouri

Thesis Title: " Design and Analysis of the Intelligent Drive System for a Five-phase Hybrid Stepping Motor "

Advisor: Dr. Zongpei Wang

\section{EMPLOYMENT HISTORY:}

1/98-Present West Virginia University, Graduate Research Assistant, Department of Computer Science and Electrical Engineering

8/97-12/97 West Virginia University, Graduate Teaching Assistant, Department of Computer Science and Electrical Engineering

11/96-3/97 Dalian Building Automation, Inc., Electrical Engineer.

4/93-7/97 Dalian Railway Institute in China, Lecturer, Department of Electrical Engineering

7/91-3/93 Harbin Institute of Technology in China, Graduate Research Assistant, Department of Electrical Engineering

\section{PUBLICATIONS IN REFERRED JOURNALS:}

[1]: William R. Cawthorne, Parviz. Famouri, J. Chen, Nigel Clark, Christopher Atkinson, Richard Atkinson, Thomas McDaniel, Subhash Nandkumar, Sorin Petreanu "Development of a Linear Alternator-Engine for Hybrid Vehicle Applications", IEEE Transactions on Vehicular Technology, Sept. 1998 vol 14, pp1797-1802

[2]: J.M.Dawson, J-D.Chen, K.S.Brown, P.Famouri, and L.A.Hornak, " Through-Wafer Interrogation of Microstructure Motion for MEMS Feedback Control", SPIE, Dec. 2000, pp3239--3246

[3]: P.Famouri, J.Chen, “ A Linear Alternator-Engine for Electrical Power Generation”, submitted to IEEE Transactions on Energy Conversion. 


\section{PUBLICATIONS IN PROCEEDINGS:}

[1]: Jingdong Chen, Parviz Famouri, Lawrence A. Hornak, " Nonlinear Control of MEMS: Microengine Sliding Mode Control Simulation", Proc. International Conference on Intelligent Systems and Control, Santa Barbara, California, October 28-30, 1999, pp96-101.

[2]: J.M.Dawson, J-D.Chen, K.S.Brown, P.Famouri, and L.A.Hornak, " Through-Wafer Interrogation of Microstructure Motion for MEMS Feedback Control", SPIE Conference on Miniaturized Systems with Micro-Optics and MEMS, Sept. 1999, SPIE, vol.3878, pp281-292

[3]: Parviz Famouri, Jingdong Chen, J. Cathy " Dynamic Analysis of a Single-phase Synchronous Reluctance Generator under Normal and Fault Operations ", submitted to Annual meeting 2000 of IEEE on Industry Application.

[4]: J. M. Dawson, L. Wang, J. Chen, P. Famouri, L. A. Hornak, "Utilization of ThroughWafer Optical Device Monitoring for MEMS Feedback and Control”, SPIE Conference on Micromachining and Microfabrication 2000,18-21 September 2000 , Santa Clara, California USA

[5]: L. Wang, J. M. Dawson, J. Chen, P. Famouri and L.A. Hornak, "Stroke-Length Control of a MEMS Device", ISIE'2000 ,The 2000 IEEE International Symposium on Industrial Electronics, 4 - 8 December, 2000

\section{PRESENTATIONS:}

Jingdong Chen, " Nonlinear Control of MEMS: Microengine Sliding Mode Control Simulation", Presented in the International Conference on Intelligent Systems and Control, Santa Barbara, California, October 28-30, 1999, pp96-101.

\section{RESEARCH INTERESTS AND PROJECTS:}

My primary research interests include design, analysis, modeling, and control of electromechanical systems, power electronics, and electric and hybrid vehicles. My objectives and long term professional goals are to pursue an academic career in effective teaching and quality research in electric machine and power system.

I have been a research assistant for three years, the projects I have involved are as following:

1: Linear Alternator/internal combustion engine combination for hybrid electric vehicle application, sponsored

by Department of Defense.

A generation system that employs a linear crankless internal combustion engine in conjunction with a linear alternator directly utilizes the linear motion of the piston to drive the alternator rather than first converting to rotary motion. The result is a more compact, reliable, and efficient unit, making the system ideal for use in series hybrid electric vehicle. In this project, my duty is to model the linear induction alternator and provide a novel approach to analyze its performances considering the nonlinearity.

2: MicroElectroMechanical Systems(MEMS) Control ,sponsored by National Science Foundation.

Intrinsic Micro-Electro-Mechanical Systems (MEMS) characteristics of small size, lightweight, and fast response give them a distinct advantage for a variety of embedded system applications given that precise, robust operation can be achieved. As a key investigator, I helped obtaining the grant by proposing a 
nonlinear sliding mode control scheme. Now, I am concerning on the system dynamic modeling, controller design and simulation, which provide a clear guidance for this challenging project.

3: Dynamic Analysis of Self-excited Reluctance Generator.

With the requirement for renewable energy, stand-alone self-excited reluctance generators have attracted more attentions for wind, tidal and hydro power generation applications. In this work, I propose a novel inductance based methodology for steady state analysis dealing with the nonlinear nature of the system. Another finite element +state space method considering saturation is also suggested for the transient analysis. These two approaches supply new tools for the study of this kind of nonlinear problems.

\section{FUND RAISING:}

"Integrated Optical Monitoring: Enabling Technology for MEMS Feedback Control", L.A. Hornak PI and P.Famouri Co-PI, NSF Phys. Found. Of Enabling Technologies, $\$ 241,997,7 / 1 / 1999-6 / 30 / 2002$

During the application of this grant, I played key role in selecting and validating effective feedback control schemes applied in Microsystems. Both linear and nonlinear control methods were proposed with special concern on dynamic response and system uncertainties.

\section{PAPER REVIEWING:}

My advisor Dr. Parviz Famouri gave me many chances to review technical papers for IEEE Transactions and Conferences, and feedback my comments to him, which, in turn, provided good exercises to me on technique evaluation.

\section{TEACHING EXPERIENCE:}

Having been a lecturer for four years and as a teaching assistant, I realize teaching is one of my favorites. When I see the brightening eyes of my students, real happiness raises from deep my heart.

Courses I have taught:

(1): Electrical Machinery.

(2): Power Electronics.

(3): Motor Control Theory.

(4): Introduction to Electric Circuits.

(5): Introduction of Relay Protection for Power System.

\section{INDUSTRIAL EXPERIENCE:}

Familiar with motion control system design.

I have developed an intelligent drive system for a five-phase hybrid stepping motor used in digital controlled lathe or embroidering machine. Two stepping motors and drive circuits are designed for two dimensional position control. Special algorithm is coded for acceleration and deceleration process to maintain good accuracy. The whole process includes understanding the system requirement, control and drive circuit design and the performance analysis. 


\title{
SKILLS:
}

1: Control Systems Background:

Completed graduate control courses including Fundamentals of Control Systems, Linear Control Systems, Optimal Control, Applied Nonlinear Control, Applied Adaptive Control, Neural Network,

2: Power Systems Background:

Completed graduate control courses including Power Electronics, Advanced Power, Electronics and Drives, Advanced Electrical Machinery, Real Time Control for Electric Power Systems.

3: Computer Background:

Proficient programming in $\mathrm{C}, \mathrm{C}++$, MATLAB, EMAS, ANSYS, MCS Assembly, Fluent with DOS, Windows 98, and Windows NT 4.0 as well as numerous software packages including finite element analysis, circuit and logic simulation, mathematical packages, word processing and Database applications.

Able to quickly learn and utilize new software and operating systems

4: Have experience in interfacing with MCS serial microprocessor based systems

\section{ACADEMIC ACTIVITY:}

Institute of Electrical and Electronics Engineers (IEEE), Student Member

\section{HONORS AND ACTIVITIES:}

1: Three outstanding undergraduate and graduate student prizes at Harbin Institute of Technology, 1987-1993.

2: Team Leader of the Chinese Student Soccer Team of West Virginia University, 19982000.

\section{HOBBIES AND INTERESTS:}

Soccer, Singing, Reading and Traveling.

\author{
REFERENCES \\ Dr. Parviz Famouri \\ Professor, CSEE \\ West Virginia University \\ Engineering Sciences Building \\ P.O. Box 6109 \\ Morgantown, WV 26506-6109 \\ (304) 293-6371 Ext. 2530 \\ Email: pfamouri@wvu.edu \\ Dr. Ronald A. Klein \\ Professor, CSEE \\ West Virginia University \\ Engineering Sciences Building \\ P.O. Box 6109 \\ Morgantown, WV 26506-6109 \\ (304) 293-6371 Ext. 2518 \\ Email: KLEIN@cemr.wvu.edu
}

\author{
Dr. Muhammad A. Choudhry \\ Professor, CSEE \\ West Virginia University \\ Engineering Sciences Building \\ P.O. Box 6109 \\ Morgantown, WV 26506-6109 \\ (304) 293-6371 Ext. 2524 \\ Email: choudhry@cemr.wvu.edu \\ Dr. Larry A. Hornak \\ Professor, CSEE \\ West Virginia University \\ Engineering Sciences Building \\ P.O. Box 6109 \\ Morgantown, WV 26506-6109 \\ (304) 293-6371 Ext. 2515 \\ Email: lah@csee.wvu.edu
}

